\title{
A unified approach to holomorphic anomaly equations and quantum spectral curves
}

\author{
Zhiyuan Wang and Jian Zhou \\ Department of Mathematical Sciences, Tsinghua University, \\ Beijing, 100084, China \\ E-mail: zhiyuan-14@mails.tsinghua.edu.cn, \\ jianzhou@mail.tsinghua.edu.cn
}

ABSTRACT: We present a unified approach to holomorphic anomaly equations and some well-known quantum spectral curves. We develop a formalism of abstract quantum field theory based on the diagrammatics of the Deligne-Mumford moduli spaces $\overline{\mathcal{M}}_{g, n}$ and derive a quadratic recursion relation for the abstract free energies in terms of the edge-cutting operators. This abstract quantum field theory can be realized by various choices of a sequence of holomorphic functions or formal power series and suitable propagators, and the realized quantum field theory can be represented by formal Gaussian integrals. Various applications are given.

Keywords: String Duality, String theory and cosmic strings, Topological Strings

ARXiv EPRINT: 1808.05343 


\section{Contents}

1 Introduction 1

2 Diagrammatics of moduli space of curves and abstract QFT 4

2.1 Moduli space of stable curves and Feynman diagrams 4

2.2 Operators on vector space associated to the set of stable graphs 5

2.3 Abstract free energy 6

2.4 A recursion relation for the abstract free energy 8

3 A generalization to labelled graphs $\quad 14$

$\begin{array}{lll}3.1 & \text { Diagrammatics for labelled graphs } & 14\end{array}$

$\begin{array}{lll}3.2 & \text { A recursion relation for the abstract free energy } & 16\end{array}$

$\begin{array}{lll}4 & \text { Realization of the abstract quantum field theory } & 17\end{array}$

$\begin{array}{lll}4.1 & \text { Realization of the abstract QFT by Feynman rules } & 17\end{array}$

$\begin{array}{lll}4.2 & \text { Representation by formal Gaussian integrals } & 19\end{array}$

4.3 Realization of the operators on stable graphs 20

$\begin{array}{lll}4.4 & \text { Realization of the recursion relations } & 22\end{array}$

$\begin{array}{ll}4.5 & \text { The general case } \\ \end{array}$

5 The holomorphic anomaly equation $\quad 24$

6 Relationship to Eynard-Orantin topological recursion and quantum spec$\begin{array}{ll}\text { tral curves } & \mathbf{2 6}\end{array}$

6.1 Graph sum for Eynard-Orantin topological recursion 26

$\begin{array}{lll}6.2 & \text { Graph sum for quantum spectral curves } & 28\end{array}$

6.3 Recursion relations for $S_{n}(z) \quad 31$

7 Application to topological 1D gravity and quantum spectral curves $\quad 33$

$\begin{array}{lll}\text { 7.1 Some results in topological 1D gravity } & 33\end{array}$

7.2 Topological 1D gravity as realization of the diagrammatics of stable graphs 34

7.3 Example: the Airy function and quantum Airy curve 37

7.4 Example: the Kontsevich-Penner matrix model for $N=1 \quad 40$

7.5 Example: enumeration of stable graphs with genus zero vertices 42

7.6 Example: enumeration of graphs 44

$\begin{array}{ll}\text { A Some explicit expressions for } \widehat{\boldsymbol{F}}_{g, n} & 50\end{array}$ 


\section{Introduction}

There are various types of quadratic recursions which have played an important role in Gromov-Witten theory. The Virasoro constraints are a sequence of recursion relations $[8,12,18]$ for the free energy defined on the big phase space. It involves differential operators of infinitely many variables. There are also quadratic recursion relations for the free energy restricted to the small phase space that involves only finitely many variables. For example, the BCOV holomorphic anomaly equation [3] and the Eynard-Orantin topological recursion relations [16]. The former was developed by Bershadsky, Cecotti, Ooguri and Vafa to compute Gromov-Witten invariants of the quintic Calabi-Yau threefold [2, 3]. The latter was first discovered by Eynard and Orantin in the setting of matrix models and later formulated in a form which is conjectured and proved to hold in general for $n$-point functions. For an approach to the holomorphic anaomaly equation from the point of view of Eynard-Orantin topological relation, see Eynard-Mariño-Orantin [17]. In the case of Witten-Kontsevich tau-function, it has been shown by the second author that the Virasoro constraints are equivalent to the EO topological recursion relations [35]. It is an interesting problem to establish a relationship between the Virasoro constraints and the EO topological relations. The basic ingredient for EO topological recursion is an algebraic curve with some extra data, called the spectral curve. A suitable quantization of the spectral curve leads to a Schrödinger equation satisfied by a partition function constructed from the EO topological recursion, called the quantum spectral curve (cf. Gukov-Sułkowski [20]). It is a natural problem to understand the relationship between the holomorphic anomaly equation and the quantum spectral curves. In this work we will present a formalism that gives a unified construction of the holomorphic anomaly equation and the wave functions for the quantum spectral curves.

To formulate the BCOV holomorphic anomaly equation, it is crucial to first extend the free energy $F_{g}(t)$ to a non-holomorphic free energy $\widehat{F}_{g}(t, \bar{t})$ whose geometric meaning is not mathematically clear in the literature. It is supposed to be modular-invariant, and involve contributions from the boundary strata of the moduli spaces. Originally the holomorphic anomaly is an equation for $\partial_{\bar{t}^{i}} \widehat{F}_{g}$ which involves $\widehat{F}_{h}$ for $h<g$ of the following form:

$$
\bar{\partial}_{\bar{t}_{i}} \widehat{F}_{g}=\frac{1}{2} \bar{C}_{\bar{i}}^{(0) j k}\left(D_{j} D_{k} \widehat{F}_{g-1}+\sum_{r=1}^{g-1} D_{j} \widehat{F}_{r} D_{k} \widehat{F}_{g-r}\right) .
$$

It is shown in [3] that this system of equations can be recursively solved to get

$$
\widehat{F}_{g}(t, \bar{t})=\Gamma^{(g)}\left(\Delta^{i j}, \Delta^{i}, \Delta, C_{i_{1}, \ldots, i_{n}}^{(r<g)}\right)+f^{(g)}(t),
$$

where $\Gamma^{(g)}$ is a polynomial equation in $\Delta^{i j}, \Delta^{i}, \Delta$ (propagators) and $C_{i_{1}, \ldots, i_{n}}^{(r<g)}$ (lower genus vertices). Furthermore, the authors of that work present some explicit expressions of $\widehat{F}_{g}$ for $g=2$ and 3 and the corresponding Feynman graphs and Feynman rules (cf. [3, (6.7), figure 17] and [3, (6.8), figure 18]). Inspired by Witten [30], Aganagic, Bouchard and Klemm $[1,(2.16)]$ obtain the following expression for $\widehat{F}_{g}$ :

$$
\widehat{F}_{g}(t, \bar{t})=F_{g}(t)+\Gamma_{g}\left(-\left((\tau-\bar{\tau})^{-1}\right)^{I J}, \partial_{I_{1}} \cdots \partial_{I_{n}} F_{r<g}(t)\right),
$$


where $\tau=\left(\tau_{i j}\right)=\left(\frac{\partial^{2} F_{0}}{\partial t_{i} \partial t_{j}}\right)$. This was interpreted for matrix models using Eynard-Orantin topological recursion by Eynard, Mariño and Orantin [17], and furthermore, they also reformulate the partition function as a formal Gaussian integral [17, (4.27)] and present the Feynman graphs and Feynman rules for the terms that contribute to $\Gamma_{g}$. These results have been generalized to other models by Grimm, Klemm, Mariño and Weiss [19]. These authors reformulate the holomorphic anomaly equation as a quadratic recursion relation for the derivative of $\widehat{F}_{g}$ with respective to the propagators $\Delta^{I J}$ (cf. [19, (7.50)]:

$$
\frac{\partial \widehat{F}_{g}}{\partial \Delta^{I J}}=\frac{1}{2} D_{I} \partial_{J} \widehat{F}_{g-1}+\frac{1}{2} \sum_{r=1}^{g-1} \partial_{I} \widehat{F}_{r} \partial_{J} \widehat{F}_{g-r} .
$$

Here the propagators $\Delta^{I J}$ has the form

$$
\Delta^{I J}=-\frac{1}{2 \sqrt{-1}}\left((\tau-\bar{\tau})^{-1}\right)^{I J}+\mathcal{E}^{I J},
$$

where $\mathcal{E}^{I J}$ is a holomorphic function. They also derive the formal Gaussian integral representation of the partition function and the Feynman expansions for $\widehat{F}_{g}$.

To summarize, one can see the common features of the above works on holomorphic anomaly equations. One starts with a sequence of functions $F_{g}(t)(g \geq 0)$ for finitely many variables $t_{1}, \ldots, t_{n}$, and seek for another sequence $\widehat{F}_{g}(t, \bar{t})$ such that $\lim _{\bar{t} \rightarrow \infty} \widehat{F}_{g}(t, \bar{t})=F_{g}(t)$, and such that the sequence $\widehat{F}_{g}(t, \bar{t})$ satisfies quadratic recursion equations of the form (1.3). Furthermore, the partition function $\widehat{Z}(t, \bar{t})=\exp \sum_{g \geq 0} \lambda^{2 g-2} \widehat{F}_{g}(t, \bar{t})$ can be represented as a formal Gaussian integral, and there are Feynman rules that express $\widehat{F}_{g}$ as a polynomial in the propagators and the derivatives of $F_{r}$ for $r \leq g$. As pointed out in [3], $\widehat{F}(t, \bar{t})$ has contributions from the degenerate Riemann surfaces, from lower boundary strata of the Deligne-Mumford moduli spaces. It turns out that the Feynman graphs that appear in the Feynman expansion of $\widehat{F}_{g}$ in the above works are just the stable graphs that index the stratification of the Deligne-Mumford moduli spaces $\overline{\mathcal{M}}_{g}$.

In the present work, we will formulate a general construction of some kind of $a b$ stract quantum field theory that has the above works on holomorphic anomaly equations as special realizations. The key ingredient in our construction is the diagrammatics for the stratification of the Deligne-Mumford moduli spaces $\overline{\mathcal{M}}_{g, n}$, using the language of the dual graphs. There are some natural operators acting on these graphs, including an edge-cutting operator $K$. We define an abstract free energy

$$
\widehat{\mathcal{F}}_{g}=\sum_{\Gamma \in \mathcal{G}_{g, 0}^{c}} \frac{1}{|\operatorname{Aut}(\Gamma)|} \Gamma
$$

and derive a quadratic relation

$$
K \widehat{\mathcal{F}}_{g}=\frac{1}{2}\left(\mathcal{D} \partial \widehat{\mathcal{F}}_{g-1}+\sum_{r=1}^{g-1} \partial \widehat{\mathcal{F}}_{r} \partial \widehat{\mathcal{F}}_{g-r}\right)
$$

for $g \geq 2$ using the operators $\partial$ and $\mathcal{D}$ which correspond to adding external edges on the graphs. For details, see section 2. For generalizations that involves more labelling on the graphs, see section 3 . 
Another ingredient in our formalism is what we call the realizations of the abstract quantum field theory, we need a sequence of functions $F_{g}(t), t=\left(t_{1}, \ldots, t_{n}\right), g \geq 0$, and a symmetric nondegenerate matrix $\kappa$ that depends on $t$ and $\bar{t}$. They will be used to give the Feynman rules that associate a weight $\omega_{\Gamma}$ to each $\Gamma$. This kind of realization of the above abstract quantum field theory can also be represented as a formal Gaussian integral. We will refer to this as the formal Gaussian integral representation of the abstract quantum field theory. These will be presented in section 4 .

Using this formalism, given a holomorphic function $F(t)=\sum \lambda^{2 g-2} F_{g}(t)$, we will obtain a family of free energies $\widehat{F}_{g}(\kappa, t)$. Here $\widehat{F}_{g}(\kappa, t)$ is a polynomial in $\kappa$ of degree $3 g-3$, whose coefficients are some differential polynomials in $\left\{F_{k}\right\}_{0 \leq k \leq g}$ for $g \geq 2$. In particular, $\widehat{F}_{g}(0, t)=F_{g}(t)$ for $g \geq 2$.

Let us now mention some applications of our formalism. First of all, for the choice $\kappa=$ $(\bar{\tau}-\tau)^{-1}$, we recover the holomorphic anomaly for matrix models [15, 17], or more generally, when the propagator $\kappa^{I J}$ takes the form of $\Delta^{I J}$ in (1.4), we recover the construction of holomorphic anomaly equation in Gromov-Witten theory as discussed in [19]. For details, see section 5. Secondly, the propagators $\kappa$ can be also chosen to be holomorphic. We will use this formalism for holomorphic propagators to reinterpret some results of the second author [34] in the theory of topological 1D gravity.

We also combine the results on graph sum representation of Eynard-Orantin recursion $[11,14,24]$ with the construction of the wave functions $Z(z)$ of quantum spectral curves [20]:

$$
\widehat{A} Z(z)=0
$$

where $Z(z)$

$$
Z(z):=\exp \left(\sum_{n=0}^{\infty} \hbar^{n-1} S_{n}(z)\right),
$$

and $S_{n}(z)$ are obtained from the spectral curve and Eynard-Orantin topological recursion [20] (see also (6.4)). We reinterpret $S_{n}$ as Feynman sum over labelled stable graphs, hence relating them to a realization of abstract QFT as developed in this work (cf. (6.17)). We present some well-known examples of quantum spectral curves [20] reexamined in our formalism.

In a subsequent work [28] we have applied our formalism to the problem of computing the orbifold Euler characteristics of $\overline{\mathcal{M}}_{g, n}$ based on the formulas for $\chi_{\text {orb }}\left(\mathcal{M}_{g, n}\right)$ of HarerZagier [21] and Penner [27]. We will deal with the case of propagators of the form (1.4) in another subsequent work that generalizes the formalism of this work [29].

The rest of this paper is arranged as follows. In section 2 we formulate the diagrammatics and derive a quadratic recursive relation for the free energies using the edge-cutting operators. In section 3 we generalize the diagrammatics to the case of labelled graphs. We give a field theory realization of the diagrammatics in section 4, where the free energies can be obtained from formal Gaussian integrals and Feynman rules. The holomorphic anomaly equation is derived in section 5 in our formalism using non-holomorphic propagators. In section 6 we write the wave function of the quantum spectral curve of Gukov-Sułkowski as a summation over stable graphs, and understand it as a particular realization of the 
abstract QFT. In section 7 we apply our formalism to topological 1D gravity and derive quadratic recursion relations for this theory. As applications, we relate the recursion relations to quantum spectral curves in some important examples. Other applications include recursion relations in enumeration problems of stable graphs and general graphs.

\section{Diagrammatics of moduli space of curves and abstract QFT}

In this section we recall the diagrammatics related to the stratification of the DeligneMumford moduli spaces $\overline{\mathcal{M}}_{g, n}$. We introduce a notion of abstract quantum field theory based on stable graphs. We define the edge-cutting operators and some other operators acting on the graphs, and use them to derive a recursive relation of the abstract free energy.

\subsection{Moduli space of stable curves and Feynman diagrams}

We first recall the stratification of the Deligne-Mumford moduli space $\overline{\mathcal{M}}_{g, n}$ of stable curves [7, 22].

The Deligne-Mumford moduli space $\overline{\mathcal{M}}_{g, n}$ is a smooth orbifold of complex dimension $3 g-3+n$. There are two types of basic maps over these spaces, namely the forgetful map

$$
\pi_{n+1}: \overline{\mathcal{M}}_{g, n+1} \rightarrow \overline{\mathcal{M}}_{g, n}
$$

which simply forgets the $(n+1)$-th marked point and contract the unstable component; and the gluing maps

$$
\begin{aligned}
\xi_{1}: \overline{\mathcal{M}}_{g_{1}, n_{1}+1} & \times \overline{\mathcal{M}}_{g_{2}, n_{2}+1} \\
\xi_{2}: \overline{\mathcal{M}}_{g-1, n+2} & \rightarrow \overline{\mathcal{M}}_{g_{1}+g_{2}, n_{1}+n_{2}}, \\
& \rightarrow \overline{\mathcal{M}}_{g, n},
\end{aligned}
$$

which glues two marked points together to produce a new nodal point. The union of images of $\xi_{1}$ and $\xi_{2}$ is the boundary $\overline{\mathcal{M}}_{g, n} \backslash \mathcal{M}_{g, n}$; moreover, it is of codimension one in $\overline{\mathcal{M}}_{g, n}$. In this way, we get a stratification of the compactified moduli space $\overline{\mathcal{M}}_{g, n}$ by decomposing it into the union of some products of the moduli spaces $\mathcal{M}_{h, m}$ of smooth stable curves.

An efficient way of describing this stratification is to use the language of graphs. Recall that for a stable curve $\left(C ; x_{1}, \cdots, x_{n}\right)$ of genus $g$ with $n$ marked points, its dual graph is defined as follows. Let $\tilde{C}$ be its normalization, then we associate a vertex to each of the connected components of $\tilde{C}$, and write down the genus of this component at this vertex. For each nodal point of $C$, we draw an internal edge connecting the corresponding vertices; and for each marked point, we draw an external edge attaching to the corresponding vertex, and write the markings $i \in\{1,2, \cdots, n\}$ besides the external edges.

In what follows we will forget all the markings of the external edges, which corresponds to the moduli space $\overline{\mathcal{M}}_{g, n} / S_{n}$ parametrizing equivalent classes of stable curves with $n$ marked points while we ignore the ordering of these marked points. In this way, we obtain a graph of genus $g$ with $n$ external edges, where the genus is defined to be the sum of the number of loops in the graphs and all the numbers associated to the vertices. 


\section{(1)- C(0)-}

Figure 1. Dual graphs for $\overline{\mathcal{M}}_{1,1}$.

\section{(2) C(1) (1)-(1) C(0) (1)-(0) C(0)-(0) (0)=(0)}

Figure 2. Dual graphs for $\overline{\mathcal{M}}_{2,0}$.

Example 2.1. All possible dual graphs of curves in $\overline{\mathcal{M}}_{1,1}$, and $\overline{\mathcal{M}}_{2,0}$ are listed in figure 1 and figure 2 respectively.

A graph $\Gamma$ is called stable if all its vertices are stable, that is, the valence of each vertex of genus 0 is at least three, and the valence of each vertex of genus 1 is at least one. It is clear that the stability of curves is equivalent to the stability of its dual graph. The stable graphs will be the Feynman graphs used in this paper.

Let $\mathcal{G}_{g, n}$ be the set of all stable graphs of genus $g$ with $n$ external edges (not necessarily connected), and $\mathcal{G}_{g, n}^{c}$ be the subset of connected stable graphs.

Now let $\Gamma$ be a stable graph of genus $g$ with $n$ external edges, and define $\mathcal{M}_{\Gamma}$ to be the subset of $\overline{\mathcal{M}}_{g, n} / S_{n}$ consisting of all equivalent classes of curves whose dual graph is $\Gamma$, then

$$
\overline{\mathcal{M}}_{g, n} / S_{n}=\bigsqcup_{\Gamma \in \mathcal{G}_{g, n}^{c}} \mathcal{M}_{\Gamma}
$$

gives the stratification of $\overline{\mathcal{M}}_{g, n} / S_{n}$.

\subsection{Operators on vector space associated to the set of stable graphs}

Denote by $\mathcal{V}^{c}$ the vector space over $\mathbb{Q}$ generated by all connected stable graphs, i.e.,

$$
\mathcal{V}^{c}=\bigoplus_{\substack{\Gamma \in \mathcal{G}_{g, n}^{c} \\ 2 g-2+n>0}} \mathbb{Q} \Gamma ;
$$

similarly, denote by $\mathcal{V}$ the vector space over $\mathbb{Q}$ generated by all stable graphs, not necessarily connected, i.e.,

$$
\mathcal{V}=\bigoplus_{\substack{\Gamma \in \mathcal{G}_{g, n} \\ 2 g-2+n>0}} \mathbb{Q} \Gamma .
$$

In this subsection we define some operators on $\mathcal{V}$. These operators may be understood as the inverse procedures of the gluing maps and forgetful maps.

Define an operator $K$ acting on $\mathcal{V}$ :

$$
K: \mathcal{V} \rightarrow \mathcal{V}, \quad \Gamma \in \mathcal{G}_{g, n} \mapsto K(\Gamma)=\sum_{\Gamma^{\prime}} \Gamma^{\prime},
$$

where the summation is over all graphs $\Gamma^{\prime}$ obtained by cutting an internal edge of $\Gamma$. We will refer to this operator as the edge cutting operator. It is easy to see that the operator $K$ does not affect the stability of the graph. It decreases the number of internal edges by 
one, and increases the number of external edges by two. In the picture of stable curves, this operator corresponds to breaking up a node and regard it as two new marked points. So the operator $K$ can be regarded as the inverse operator of the gluing maps $\xi_{1}$ and $\xi_{2}$.

\section{Example 2.2.}

$$
\begin{aligned}
& K(2)=0, \\
& K C(0)-(0)=2 C(0)-(0)+C(0)-(0), \\
& K(0)=(0)=3-\text { (0) (0) } .
\end{aligned}
$$

Let us define another operator $\partial$. This operator has two parts, one is to attach an external edge to a vertex and sum over all vertices, and the other is to break up an internal edge and insert a stable vertex of genus 0 with three edges.

\section{Example 2.3.}

$$
\begin{aligned}
& \partial \text { (1) }=-1) \\
& \text { z (0)-(0) }=2 \text { C(0)-(0) }+2 \text { C(0)-(0)-(0)- + (0)-(0)-(0) } \\
& \partial \quad(0)=(1)=-(0)=(1)+(0)=(1)-+3 \stackrel{1}{0}=(1) \text {. }
\end{aligned}
$$

In the dual picture, the operator $\partial$ is just adding one marked point, or breaking up a node of the curve and gluing a 3-pointed sphere.

Now we define a third operator $\gamma$, which acts on $\Gamma$ by simply attaching a stable vertex of genus 0 with three edges to an external edge of $\Gamma$, and summing over all external edges.

\section{Example 2.4.}

$$
\begin{aligned}
& \gamma \text { (1)- }=\text { (1)-(0) } \\
& \gamma \text { c(0) }=2 \text { (C)-(0). }
\end{aligned}
$$

If the graph $\Gamma$ has no external edge, then we may simply have $\gamma(\Gamma)=0$. This operator is to glue a 3-pointed sphere to the curve along marked points to get a new node.

Clearly the operators $\partial$ and $\gamma$ also preserves the stability, and they both increase the number of external edges by one. Intuitively, $\partial+\gamma$ is the inverse of the forgetful map. Write $\mathcal{D}=\partial+\gamma$. Then $\mathcal{D}$ preserves the subspaces $\mathcal{V}^{c}$.

\subsection{Abstract free energy}

Let us now define an abstract quantum field theory based on the diagrammatics of stable graphs discussed above.

Definition 2.1. For $g \geq 2$, we define the abstract free energy of genus $g$ to be

$$
\widehat{\mathcal{F}}_{g}=\sum_{\Gamma \in \mathcal{G}_{g, 0}^{c}} \frac{1}{|\operatorname{Aut}(\Gamma)|} \Gamma .
$$


In general, for $2 g-2+n>0$, define the abstract $n$-point function of genus $g$ to be

$$
\widehat{\mathcal{F}}_{g, n}=\sum_{\Gamma \in \mathcal{G}_{g, n}^{c}} \frac{1}{|\operatorname{Aut}(\Gamma)|} \Gamma,
$$

then the free energy $\widehat{\mathcal{F}}_{g}$ is just $\widehat{\mathcal{F}}_{g, 0}$.

Example 2.5. Here we list some explicit expressions of $\widehat{\mathcal{F}}_{g, n}$ for small $(g, n)$.

$$
\begin{aligned}
& \widehat{\mathcal{F}}_{0,3}=\frac{1}{6}-\text { - } 0 \text {, } \\
& \widehat{\mathcal{F}}_{0,4}=\frac{1}{24} \text { 二(0) }+\frac{1}{8} \text { 二0-(0), }
\end{aligned}
$$

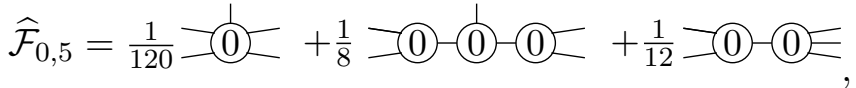

$$
\begin{aligned}
& \widehat{\mathcal{F}}_{1,1}=(1)-+\frac{1}{2} \subset(0)- \\
& \widehat{\mathcal{F}}_{1,2}=\frac{1}{2}-(1)-+\frac{1}{4} \text { C(0) }+\frac{1}{2} \text { (1)-(0) }+\frac{1}{4} \text { C(0)-(0) }+\frac{1}{4}-\text { (0)=(0)- }
\end{aligned}
$$

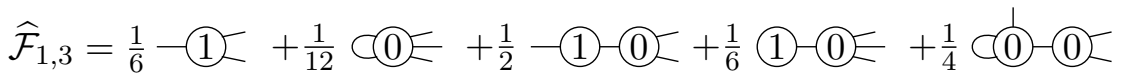

$$
\begin{aligned}
& +\frac{1}{4} \text {-(0)-(0) }+\frac{1}{12} \text { C(0)-(0) E + }+\frac{1}{2} \text { (1)-(0)-(0) + }+\frac{1}{4} \text {-(0)-(0)-(0) } \\
& +\frac{1}{4} \text { (0)-(0)-(0) }+\frac{1}{6} \text {-(10)(0), } \\
& \widehat{\mathcal{F}}_{2,0}=(2)+\frac{1}{2} C(1)+\frac{1}{2} \text { (1)-(1) }+\frac{1}{8}\left(\text { (0) }+\frac{1}{2} \text { (1)-(0) }+\frac{1}{8} \text { C(0)-(0) }+\frac{1}{12}\right. \text { (1)=(0) } \\
& \widehat{\mathcal{F}}_{2,1}=(2)-+\frac{1}{2} C(1)-+(1)-(1)-+\frac{1}{8} C(0)+\frac{1}{2}-(1)-(0)+\frac{1}{2} \text { (1)-(0)- } \\
& +\frac{1}{2} \text { (1)-(0) }+\frac{1}{2} \text { (1)-(0)-(1) + } \frac{1}{4} \text { (C)-(0) }+\frac{1}{4}-(0)=(0) \\
& +\frac{1}{6} \text { (1)=(0)- }+\frac{1}{2} \text { C(0)-(0)-(1) }+\frac{1}{2}-\text { (1)-(0)-(1) }+\frac{1}{4}-\text { (1)-(0)-(0) } \\
& +\frac{1}{8} \text { C(0)-(0)-(0) }+\frac{1}{4} \text { (0) } \stackrel{(0)}{=} \text { (0). }
\end{aligned}
$$

More examples will be given in appendix A. 


\subsection{A recursion relation for the abstract free energy}

Example 2.6. Using the expressions in the above example, one can directly check the following identities:

$$
\begin{aligned}
K \widehat{\mathcal{F}}_{0,4} & =\frac{9}{2}\left(\widehat{\mathcal{F}}_{0,3}\right)^{2}, \\
K \widehat{\mathcal{F}}_{0,5} & =12 \widehat{\mathcal{F}}_{0,3} \widehat{\mathcal{F}}_{0,4}, \\
K \widehat{\mathcal{F}}_{1,1} & =3 \widehat{\mathcal{F}}_{0,3} \\
K \widehat{\mathcal{F}}_{1,2} & =6 \widehat{\mathcal{F}}_{0,4}+3 \widehat{\mathcal{F}}_{1,1} \widehat{\mathcal{F}}_{0,3}, \\
K \widehat{\mathcal{F}}_{1,3} & =10 \widehat{\mathcal{F}}_{0,5}+4 \widehat{\mathcal{F}}_{1,1} \widehat{\mathcal{F}}_{0,4}+6 \widehat{\mathcal{F}}_{1,2} \widehat{\mathcal{F}}_{0,3}, \\
K \widehat{\mathcal{F}}_{2} & =\widehat{\mathcal{F}}_{1,2}+\frac{1}{2}\left(\widehat{\mathcal{F}}_{1,1}\right)^{2}, \\
K \widehat{\mathcal{F}}_{2,1} & =3 \widehat{\mathcal{F}}_{1,3}+2 \widehat{\mathcal{F}}_{1,1} \widehat{\mathcal{F}}_{1,2} .
\end{aligned}
$$

In general, we have the following relation.

Theorem 2.1. For $2 g-2+n>0$, we have

$$
K \widehat{\mathcal{F}}_{g, n}=\left(\begin{array}{c}
n+2 \\
2
\end{array}\right) \widehat{\mathcal{F}}_{g-1, n+2}+\frac{1}{2} \sum_{\substack{g_{1}+g_{2}=g, n_{1}+n_{2}=n+2, n_{1} \geq 1, n_{2} \geq 1}}\left(n_{1} \widehat{\mathcal{F}}_{g_{1}, n_{1}}\right)\left(n_{2} \widehat{\mathcal{F}}_{g_{2}, n_{2}}\right)
$$

where the sum on the right hand side is taken over all stable cases.

Proof. First note that cutting off an internal edge of a stable graph in $\mathcal{G}_{g, n}^{c}$ gives us two types of new stable graphs, one type is still connected, and must be of genus $g-1$ with $n+2$ external edges; the other type of graphs have two connected components, and have total genus $g$ with $n+2$ external edges. Thus we write

$$
K \widehat{\mathcal{F}}_{g, n}=I_{g, n}+J_{g, n}
$$

where $I_{g, n}$ and $J_{g, n}$ are sums over connected graphs and disconnected graphs respectively.

We already know that graphs appearing in $I_{g, n}$ all belong to $\mathcal{G}_{g-1, n+2}^{c}$; conversely, every graph in $\mathcal{G}_{g-1, n+2}^{c}$ may be transformed to a graph appearing in $\widehat{\mathcal{F}}_{g, n}$ once we glue two external edges together. This tells us that the graphs appearing in $I_{g, n}$ are the exactly the same graphs in $\widehat{\mathcal{F}}_{g-1, n+2}$. Now we check the coefficients of every graph in the two sums coincide. For a stable graph $\Gamma \in \mathcal{G}_{g-1, n+2}^{c}$, there are $\left(\begin{array}{c}n+2 \\ 2\end{array}\right)$ ways to choose two external edges to be glued together. Denote by $\widetilde{\Gamma}$ one of the equivalence classes of graphs obtained from $\Gamma$ by gluing two external edges. Then we need to show that

$$
K^{\prime}\left(\sum_{\widetilde{\Gamma} \in \mathcal{G}_{\Gamma}} \frac{1}{|\operatorname{Aut}(\widetilde{\Gamma})|} \cdot \widetilde{\Gamma}\right)=\left(\begin{array}{c}
n+2 \\
2
\end{array}\right) \cdot \frac{1}{|\operatorname{Aut}(\Gamma)|} \Gamma,
$$

where $\mathcal{G}_{\Gamma}$ is the set of equivalence classes of stable graphs $\widetilde{\Gamma}$ such that one can obtain $\Gamma$ by cutting an edge of $\widetilde{\Gamma}$, and $K^{\prime}(\widetilde{\Gamma})=N(\widetilde{\Gamma}) \cdot \Gamma$, where $N(\widetilde{\Gamma})$ is the number of ways to cut an 


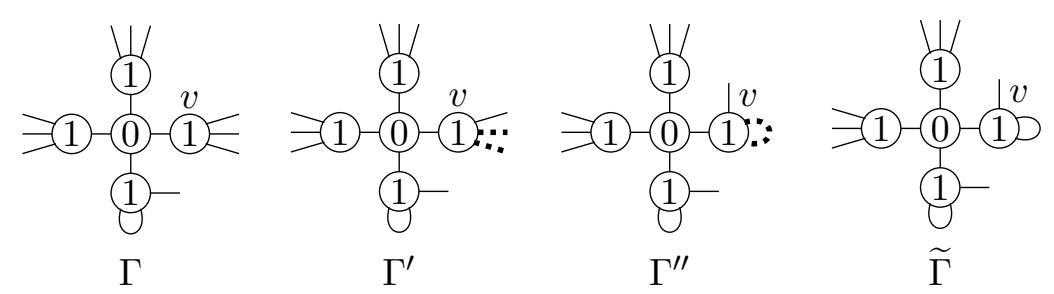

Figure 3. An example where the dotted edge is a loop.
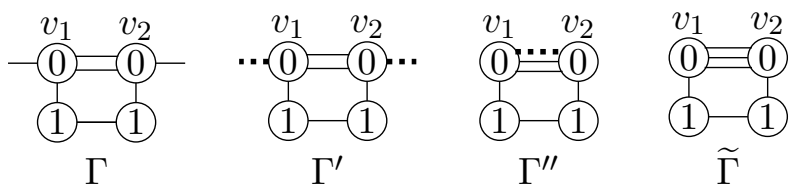

Figure 4. An example where the dotted edge is not a loop.

edge of $\widetilde{\Gamma}$ to get $\Gamma$. It suffices to show that

$$
\sum_{\widetilde{\Gamma} \in \mathcal{G}_{\Gamma}} N(\widetilde{\Gamma}) \cdot \frac{|\operatorname{Aut}(\Gamma)|}{|\operatorname{Aut}(\widetilde{\Gamma})|}=\left(\begin{array}{c}
n+2 \\
2
\end{array}\right) .
$$

Consider the set of the choices of picking a pair of external edges of $\Gamma$. This set has $\left(\begin{array}{c}n+2 \\ 2\end{array}\right)$ elements. We partition this set into disjoint union of $H_{\widetilde{\Gamma}}$ consisting of those choices for which one can get $\widetilde{\Gamma}$ by gluing the pair of external edges, where $\widetilde{\Gamma}$ runs over the set $\mathcal{G}_{\Gamma}$ of all possibilities. Therefore,

$$
\sum_{\widetilde{\Gamma} \in \mathcal{G}_{\Gamma}}\left|H_{\widetilde{\Gamma}}\right|=\left(\begin{array}{c}
n+2 \\
2
\end{array}\right) .
$$

Now we show that

$$
\left|H_{\widetilde{\Gamma}}\right|=N(\widetilde{\Gamma}) \cdot \frac{|\operatorname{Aut}(\Gamma)|}{|\operatorname{Aut}(\widetilde{\Gamma})|}
$$

or equivalently,

$$
\frac{\left|H_{\widetilde{\Gamma}}\right|}{|\operatorname{Aut}(\Gamma)|}=\frac{N(\widetilde{\Gamma})}{|\operatorname{Aut}(\widetilde{\Gamma})|}
$$

Let $\Gamma^{\prime \prime}$ be the graph obtained from $\widetilde{\Gamma}$ by changing the internal edge that we cut to get $\Gamma$ into a dotted edge, and let $\Gamma^{\prime}$ be the graph with two dotted external edges obtained from $\Gamma^{\prime \prime}$ by cutting the dotted edge. For examples, see figure 3 and figure 4 .

When we talk about automorphisms of $\Gamma^{\prime}$ and $\Gamma^{\prime \prime}$, the dotted edges can not be mapped to solid edges. Now we claim:

$$
\frac{\left|H_{\widetilde{\Gamma}}\right|}{|\operatorname{Aut}(\Gamma)|}=\frac{1}{\left|\operatorname{Aut}\left(\Gamma^{\prime}\right)\right|}=\frac{1}{\left|\operatorname{Aut}\left(\Gamma^{\prime \prime}\right)\right|}=\frac{N(\widetilde{\Gamma})}{|\operatorname{Aut}(\widetilde{\Gamma})|} .
$$

The second equality is trivial. We only need to consider the first and third equalities. Recall that $\left|H_{\widetilde{\Gamma}}\right|$ is the number of ways to choose two external edges of $\Gamma$ to be glued 
together to get $\widetilde{\Gamma}$, thus $\left|H_{\widetilde{\Gamma}}\right|$ equals to the number of all possible ways to change a pair of external edges of $\Gamma$ to dotted edges to obtain $\Gamma^{\prime}$. Similarly, $N(\widetilde{\Gamma})$ equals to the number of all possible ways to change an internal edge of $\widetilde{\Gamma}$ to a dotted edge to obtain $\Gamma^{\prime \prime}$.

In the case where the dotted edge is a loop in $\Gamma^{\prime \prime}$, denote by $v$ the vertex where it is incident to. Then let $\operatorname{Aut}_{v}(\Gamma)$ be the group of automorphisms of $\Gamma$ that fix the vertex $v$ (e.g., for figure $\left.3, \operatorname{Aut}_{v}(\Gamma) \cong\left(S_{3}\right)^{3} \times\left(S_{2}\right)^{2}\right)$. Let $O_{v}$ be the orbit of $v$ in $\Gamma$ under Aut $(\Gamma)$, then

$$
\frac{|\operatorname{Aut}(\Gamma)|}{\left|\operatorname{Aut}_{v}(\Gamma)\right|}=\left|O_{v}\right|
$$

It is clear that any automorphism of $\Gamma^{\prime}$ fixes the vertex $v$ since there is no other vertex with dotted half edges, and so $\operatorname{Aut}\left(\Gamma^{\prime}\right)$ is a subgroup of $\operatorname{Aut}_{v}(\Gamma)$. To compute the index of this subgroup, it suffices to consider the actions of these automorphisms groups on the external half edges incident at $v$. Assume there are $l$ external edges attaching to $v$ in $\Gamma^{\prime}$ including two dotted edges, then we have

$$
\frac{\left|\operatorname{Aut}_{v}(\Gamma)\right|}{\left|\operatorname{Aut}\left(\Gamma^{\prime}\right)\right|}=\frac{l !}{(l-2) !} \cdot \frac{1}{2},
$$

here the factor $\frac{1}{2}$ comes from the symmetry between two dotted edges. Now (2.15) and (2.16) give us

$$
\frac{|\operatorname{Aut}(\Gamma)|}{\left|\operatorname{Aut}\left(\Gamma^{\prime}\right)\right|}=\left|O_{v}\right| \cdot\left(\begin{array}{l}
l \\
2
\end{array}\right)
$$

Noticing that $\left|O_{v}\right|$ equals to the number of ways to choose the vertex $v$, and $\left(\begin{array}{l}l \\ 2\end{array}\right)$ is the number of ways to choose two external edges on $v$, thus

$$
\left|O_{v}\right| \cdot\left(\begin{array}{l}
l \\
2
\end{array}\right)=\left|H_{\widetilde{\Gamma}}\right|
$$

and this proves the first equality in (2.14).

Using the same argument, we now consider $\operatorname{Aut}_{v}(\widetilde{\Gamma})$, i.e., the group of automorphisms of $\widetilde{\Gamma}$ that fix $v$ (e.g., for figure $\left.3 \operatorname{Aut}_{v}(\widetilde{\Gamma}) \cong\left(S_{3}\right)^{2} \times\left(S_{2}\right)^{3}\right)$. Let $\widetilde{O}_{v}$ be the orbit of $v$ in $\widetilde{\Gamma}$ under $\operatorname{Aut}(\widetilde{\Gamma})$, then

$$
\frac{|\operatorname{Aut}(\widetilde{\Gamma})|}{\left|\operatorname{Aut}_{v}(\widetilde{\Gamma})\right|}=\left|\widetilde{O}_{v}\right|
$$

Assume there are $k$ loops attaching to $v$ in $\Gamma^{\prime \prime}$ including a dotted one, then

$$
\frac{\left|\operatorname{Aut}_{v}(\widetilde{\Gamma})\right|}{\left|\operatorname{Aut}\left(\Gamma^{\prime \prime}\right)\right|}=\frac{k ! \cdot 2^{k}}{(k-1) ! \cdot 2^{k-1}} \cdot \frac{1}{2}=k .
$$

Thus (2.18) and (2.19) give us

$$
\frac{|\operatorname{Aut}(\widetilde{\Gamma})|}{\left|\operatorname{Aut}\left(\Gamma^{\prime \prime}\right)\right|}=k \cdot\left|\widetilde{O}_{v}\right|,
$$

and the right hand side equals to $N(\widetilde{\Gamma})$. Therefore the third equality in (2.14) also holds. 
The case where the dotted edge in $\Gamma^{\prime \prime}$ is not a loop can be proved similarly. Let $v=\left(v_{1}, v_{2}\right)$ be the ordered pair of end points of the dotted edge. As above, we consider $\operatorname{Aut}_{v}(\Gamma)$ (here the order of $\left(v_{1}, v_{2}\right)$ should be preserved, e.g., for figure $\left.4, \operatorname{Aut}_{v}(\Gamma) \cong S_{2}\right)$, and the orbit $O_{v}$ of the ordered pair $v$ in $V(\Gamma) \times V(\Gamma)$ under $\operatorname{Aut}(\Gamma)$. We still have the equality:

$$
\frac{|\operatorname{Aut}(\Gamma)|}{\left|\operatorname{Aut}_{v}(\Gamma)\right|}=\left|O_{v}\right|
$$

Assume there are $l_{i}$ external edges attached to $v_{i}$ in $\Gamma^{\prime}$, then

$$
\frac{\left|\operatorname{Aut}_{v}(\Gamma)\right|}{\left|\operatorname{Aut}\left(\Gamma^{\prime}\right)\right|}=\frac{l_{1} !}{\left(l_{1}-1\right) !} \cdot \frac{l_{2} !}{\left(l_{2}-1\right) !} \cdot \delta=l_{1} l_{2} \cdot \delta
$$

here $\delta=\frac{1}{2}$ if $\left(v_{2}, v_{1}\right) \in O_{v}$, and $\delta=1$ if $\left(v_{2}, v_{1}\right) \notin O_{v}$. The equations (2.20) and (2.21) give us

$$
\frac{|\operatorname{Aut}(\Gamma)|}{\left|\operatorname{Aut}\left(\Gamma^{\prime}\right)\right|}=l_{1} l_{2}\left|O_{v}\right| \cdot \delta
$$

which equals $\left|H_{\widetilde{\Gamma}}\right|$.

Similarly define $\operatorname{Aut}_{v}(\widetilde{\Gamma})$ to be the group of automorphisms of $\widetilde{\Gamma}$ that fix $v$ (e.g., for figure 4 , Aut $\left.(\widetilde{\Gamma}) \cong S_{3}\right)$. Let $\widetilde{O}_{v}$ be the orbit of $v$ in $V(\widetilde{\Gamma}) \times V(\widetilde{\Gamma})$ under $\operatorname{Aut}(\widetilde{\Gamma})$, then

$$
\frac{|\operatorname{Aut}(\widetilde{\Gamma})|}{\left|\operatorname{Aut}_{v}(\widetilde{\Gamma})\right|}=\left|\widetilde{O}_{v}\right|
$$

Assume there are $k$ internal edges connecting $v_{1}$ and $v_{2}$ in $\Gamma^{\prime \prime}$ including a dotted one, then

$$
\frac{\left|\operatorname{Aut}_{v}(\widetilde{\Gamma})\right|}{\left|\operatorname{Aut}\left(\Gamma^{\prime \prime}\right)\right|}=\frac{k !}{(k-1) !} \cdot \delta=k \cdot \delta .
$$

Then the equations (2.23) and (2.24) imply

$$
\frac{|\operatorname{Aut}(\widetilde{\Gamma})|}{\left|\operatorname{Aut}\left(\Gamma^{\prime \prime}\right)\right|}=k\left|\widetilde{O}_{v}\right| \cdot \delta
$$

which equals to $N(\widetilde{\Gamma})$. Therefore the first and third equalities in (2.14) also hold in this case.

In summary, we've proved the identity

$$
I_{g, n}=\left(\begin{array}{c}
n+2 \\
2
\end{array}\right) \widehat{\mathcal{F}}_{g-1, n+2} .
$$

The relation

$$
J_{g, n}=\frac{1}{2} \sum_{\substack{g_{1}+g_{2}=g, n_{1}+n_{2}=n+2, n_{1} \geq 1, n_{2} \geq 1}}\left(n_{1} \widehat{\mathcal{F}}_{g_{1}, n_{1}}\right)\left(n_{2} \widehat{\mathcal{F}}_{g_{2}, n_{2}}\right)
$$

can be established in a similar fashion. For two graphs $\Gamma_{1} \in \mathcal{G}_{g_{1}, n_{1}}^{c}, \Gamma_{2} \in \mathcal{G}_{g_{2}, n_{2}}^{c}$, denote by $\widetilde{\Gamma}$ one of the equivalence classes of graphs obtained from $\Gamma_{1}$ and $\Gamma_{2}$ by gluing two external edges, one from each graph. Then we need to show that

$$
K^{\prime \prime}\left(\sum_{\widetilde{\Gamma} \in \mathcal{G}_{\Gamma}^{\prime \prime}} \frac{1}{|\operatorname{Aut}(\widetilde{\Gamma})|} \cdot \widetilde{\Gamma}\right)=\frac{n_{1}}{\left|\operatorname{Aut}\left(\Gamma_{1}\right)\right|} \Gamma_{1} \cdot \frac{n_{2}}{\left|\operatorname{Aut}\left(\Gamma_{2}\right)\right|} \Gamma_{2},
$$


where $\mathcal{G}_{\Gamma}^{\prime \prime}$ is the set of equivalence classes of stable graphs $\widetilde{\Gamma}$ such that one can obtain $\Gamma_{1}$ and $\Gamma_{2}$ by cutting an edge of $\widetilde{\Gamma}$, and

$$
K^{\prime \prime}(\widetilde{\Gamma})=N^{\prime \prime}(\widetilde{\Gamma}) \cdot \Gamma_{1} \Gamma_{2}
$$

where $N^{\prime \prime}(\widetilde{\Gamma})$ is the number of ways to cut an edge of $\widetilde{\Gamma}$ to get $\Gamma_{1}$ and $\Gamma_{2}$. It suffices to show that

$$
\sum_{\widetilde{\Gamma} \in \mathcal{G}_{\Gamma}^{\prime \prime}} N^{\prime \prime}(\widetilde{\Gamma}) \cdot \frac{1}{|\operatorname{Aut}(\widetilde{\Gamma})|}=\frac{n_{1}}{\left|\operatorname{Aut}\left(\Gamma_{1}\right)\right|} \cdot \frac{n_{2}}{\left|\operatorname{Aut}\left(\Gamma_{2}\right)\right|} .
$$

Consider the set of the choices of picking one external edge from each of $\Gamma_{1}$ and $\Gamma_{2}$. This set has $n_{1} n_{2}$ elements. We partition this set into disjoint union of $H_{\widetilde{\Gamma}}^{\prime \prime}$ consisting of those choices for which one can get $\widetilde{\Gamma}$ by gluing the pair of external edges, where $\widetilde{\Gamma}$ runs over the set $\mathcal{G}_{\Gamma}^{\prime \prime}$ of all possibilities. Therefore,

$$
\sum_{\widetilde{\Gamma} \in \mathcal{G}_{\Gamma}^{\prime \prime}}\left|H_{\widetilde{\Gamma}}^{\prime \prime}\right|=n_{1} n_{2}
$$

Now we show that

$$
\left|H_{\widetilde{\Gamma}}^{\prime \prime}\right|=N^{\prime \prime}(\widetilde{\Gamma}) \cdot \frac{\left|\operatorname{Aut}\left(\Gamma_{1}\right)\right| \cdot\left|\operatorname{Aut}\left(\Gamma_{2}\right)\right|}{|\operatorname{Aut}(\widetilde{\Gamma})|},
$$

or equivalently,

$$
\frac{\left|H_{\widetilde{\Gamma}}^{\prime \prime}\right|}{\left|\operatorname{Aut}\left(\Gamma_{1}\right)\right| \cdot\left|\operatorname{Aut}\left(\Gamma_{2}\right)\right|}=\frac{N^{\prime \prime}(\widetilde{\Gamma})}{|\operatorname{Aut}(\widetilde{\Gamma})|} .
$$

Let $\Gamma^{\prime \prime}$ be the graph obtained from $\widetilde{\Gamma}$ by changing the internal edge that we cut to get $\Gamma$ into a dotted edge, and $\Gamma^{\prime}$ be the disconnected graph obtained from $\Gamma^{\prime \prime}$ by cutting the dotted edge. Then the equality

$$
\frac{\left|H_{\widetilde{\Gamma}}^{\prime \prime}\right|}{\left|\operatorname{Aut}\left(\Gamma_{1}\right)\right| \cdot\left|\operatorname{Aut}\left(\Gamma_{2}\right)\right|}=\frac{1}{\left|\operatorname{Aut}\left(\Gamma^{\prime}\right)\right|}=\frac{1}{\left|\operatorname{Aut}\left(\Gamma^{\prime \prime}\right)\right|}=\frac{N^{\prime \prime}(\widetilde{\Gamma})}{|\operatorname{Aut}(\widetilde{\Gamma})|}
$$

can be proved using exactly the same method as the proof of (2.14). Suppose that the two external edges to be glued together are incident at $v_{1}$ in $\Gamma_{1}$ and $v_{2}$ in $\Gamma_{2}$ respectively.

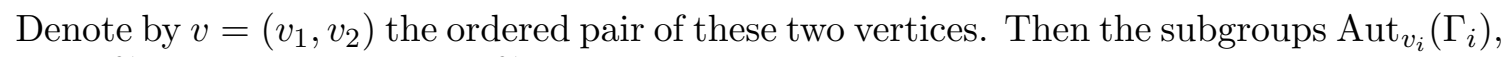
$\operatorname{Aut}_{v}(\widetilde{\Gamma})$ and the orbits $O_{v_{i}}, \widetilde{O}_{v}$ are similarly defined. Then the following equalities hold:

$$
\begin{array}{rlrl}
\frac{\left|\operatorname{Aut}\left(\Gamma_{i}\right)\right|}{\left|\operatorname{Aut}_{v_{i}}\left(\Gamma_{i}\right)\right|} & =\left|O_{v_{i}}\right|, & \frac{\left|\operatorname{Aut}_{v_{1}}\left(\Gamma_{1}\right)\right| \cdot\left|\operatorname{Aut}_{v_{1}}\left(\Gamma_{1}\right)\right|}{\left|\operatorname{Aut}\left(\Gamma^{\prime}\right)\right|}=\delta^{\prime \prime}, \\
\frac{|\operatorname{Aut}(\widetilde{\Gamma})|}{\left|\operatorname{Aut}_{v}(\widetilde{\Gamma})\right|}=\left|\widetilde{O}_{v}\right|, & \frac{\left|\operatorname{Aut}_{v}(\widetilde{\Gamma})\right|}{\left|\operatorname{Aut}\left(\Gamma^{\prime \prime}\right)\right|}=\delta^{\prime \prime},
\end{array}
$$

where $\delta^{\prime \prime}=\frac{1}{2}$ if $\Gamma_{1}=\Gamma_{2}$ and $v_{1} \in O_{v_{2}}$ under this identification, and $\delta^{\prime \prime}=1$ otherwise. Furthermore, we have

$$
\left|O_{v_{1}}\right| \cdot\left|O_{v_{2}}\right| \cdot \delta^{\prime \prime}=\left|H_{\widetilde{\Gamma}}^{\prime \prime}\right|, \quad\left|\widetilde{O}_{v}\right| \cdot \delta^{\prime \prime}=N^{\prime \prime}(\widetilde{\Gamma}) .
$$

These prove (2.32), therefore the relation (2.26) holds.

The equalities (2.8), (2.25), (2.26) complete the proof of the theorem. 
Furthermore, the right hand side of (2.7) can be rewritten in terms of operators $\mathcal{D}$. To explain this we need the following observation.

Lemma 2.1. For $2 g-2+n>0$, we have $\mathcal{D} \widehat{\mathcal{F}}_{g, n}=(n+1) \widehat{\mathcal{F}}_{g, n+1}$.

Proof. The proof is similar to the proof of Theorem 2.1. First the graphs appearing in the two sums are the same, since we've already taken all possible unstable contractions into consideration in the definition of the operator $\mathcal{D}$. Thus we only need to check the coefficients.

Let $\Gamma \in \mathcal{G}_{g, n+1}^{c}$ be a stable graph, and $\mathcal{G}_{\Gamma}$ be the subset of $\mathcal{G}_{g, n}^{c}$ consisting of graphs obtained from $\Gamma$ by removing an external edge and stabilizing. We only need to show

$$
\mathcal{D}^{\prime}\left(\sum_{\widetilde{\Gamma} \in \mathcal{G}_{\Gamma}} \frac{1}{|\operatorname{Aut}(\widetilde{\Gamma})|} \widetilde{\Gamma}\right)=(n+1) \cdot \frac{1}{|\operatorname{Aut}(\Gamma)|} \Gamma,
$$

where $D^{\prime}(\Gamma)=N(\widetilde{\Gamma}) \cdot \Gamma$ with $N(\widetilde{\Gamma})$ the number of ways to obtain $\Gamma$ from $\tilde{\Gamma}$ via one of the operations in $\mathcal{D}=\partial+\gamma$. Then it suffices to show

$$
\sum_{\widetilde{\Gamma} \in \mathcal{G}_{\Gamma}} N(\widetilde{\Gamma}) \cdot \frac{|\operatorname{Aut}(\Gamma)|}{|\operatorname{Aut}(\widetilde{\Gamma})|}=n+1 .
$$

Consider the set of the choices of picking an external edge of $\Gamma$, and partition it to subsets $H_{\widetilde{\Gamma}}$ consisting of those choices for which one can obtain $\widetilde{\Gamma}$ after removing this edge and stabilizing. Clearly

$$
\sum_{\widetilde{\Gamma} \in \mathcal{G}_{\Gamma}}\left|H_{\widetilde{\Gamma}}\right|=\left(\begin{array}{c}
n+1 \\
1
\end{array}\right)=n+1 .
$$

Now we show that

$$
N(\widetilde{\Gamma}) \cdot \frac{|\operatorname{Aut}(\Gamma)|}{|\operatorname{Aut}(\widetilde{\Gamma})|}=\left|H_{\widetilde{\Gamma}}\right|
$$

or equivalently,

$$
\frac{\left|H_{\widetilde{\Gamma}}\right|}{|\operatorname{Aut}(\Gamma)|}=\frac{N(\widetilde{\Gamma})}{|\operatorname{Aut}(\widetilde{\Gamma})|} .
$$

Denote by $\Gamma^{\prime}$ the graph obtained from $\Gamma$ by changing the external edge that we remove to a dotted edge. We will show that

$$
\frac{\left|H_{\widetilde{\Gamma}}\right|}{|\operatorname{Aut}(\Gamma)|}=\frac{1}{\left|\operatorname{Aut}\left(\Gamma^{\prime}\right)\right|}=\frac{N(\widetilde{\Gamma})}{|\operatorname{Aut}(\widetilde{\Gamma})|} .
$$

Let $v$ be the vertex of $\Gamma$ and $\Gamma^{\prime}$ that the dotted edge attaches to. The first equality follows from

$$
\begin{aligned}
\frac{|\operatorname{Aut}(\Gamma)|}{\left|\operatorname{Aut}_{v}(\Gamma)\right|} & =\left|O_{v}\right|, \quad \frac{\left|\operatorname{Aut}_{v}(\Gamma)\right|}{\left|\operatorname{Aut}\left(\Gamma^{\prime}\right)\right|}=\frac{k !}{(k-1) !} \\
\left|H_{\widetilde{\Gamma}}\right| & =\left(\begin{array}{c}
k \\
1
\end{array}\right) \cdot\left|O_{v}\right|
\end{aligned}
$$


where $\operatorname{Aut}_{v}(\Gamma)$ is the set of automorphisms of $\Gamma$ that preserve $v, O_{v}$ is the orbit of $v$ in $\Gamma$ under $\operatorname{Aut}(\Gamma)$, and $k$ is the number of external edges attaching to $v$ in $\Gamma$.

Let $\tilde{v}$ be the corresponding vertex of $v$ in $\widetilde{\Gamma}$, that is, $\tilde{v}=v$ if the graph is still stable after removing the dotted edge, and $\tilde{v}$ the vertex that $v$ contracts to if the graph is unstable. The second equality in (2.34) follows from

$$
\begin{aligned}
\frac{|\operatorname{Aut}(\widetilde{\Gamma})|}{\left|\operatorname{Aut}_{\tilde{v}}(\widetilde{\Gamma})\right|} & =\left|\widetilde{O}_{\tilde{v}}\right|, \quad\left|\operatorname{Aut}_{\tilde{v}}(\widetilde{\Gamma})\right|=\left|\operatorname{Aut}\left(\Gamma^{\prime}\right)\right| ; \\
N(\widetilde{\Gamma}) & =\left|\widetilde{O}_{\tilde{v}}\right|,
\end{aligned}
$$

where $\operatorname{Aut}_{\tilde{v}}(\widetilde{\Gamma})$ is the set of automorphisms of $\widetilde{\Gamma}$ that preserve $\tilde{v}$, and $\widetilde{O}_{\tilde{v}}$ is the orbit of $\tilde{v}$ in $\widetilde{\Gamma}$ under $\operatorname{Aut}(\widetilde{\Gamma})$.

Therefore the equality (2.34) holds, which proves the lemma.

Even though so far we have not defined $\widehat{\mathcal{F}}_{1}, \widehat{\mathcal{F}}_{0,2}$, and $\widehat{\mathcal{F}}_{0,1}$, it will be convenient for later use to define $\mathcal{D} \widehat{\mathcal{F}}_{1}, \mathcal{D} \widehat{\mathcal{F}}_{0,2}, \mathcal{D D} \widehat{\mathcal{F}}_{0,1}$ by formally applying Lemma 2.1 as follows:

$$
\begin{aligned}
\mathcal{D} \widehat{\mathcal{F}}_{1} & :=\widehat{\mathcal{F}}_{1,1}, \\
\mathcal{D} \widehat{\mathcal{F}}_{0,2} & :=3 \widehat{\mathcal{F}}_{0,3}, \\
\mathcal{D D} \widehat{\mathcal{F}}_{0,1}: & :=6 \widehat{\mathcal{F}}_{0,3} .
\end{aligned}
$$

Now using Lemma 2.1 and conventions in (2.35), our Theorem 2.1 can be rewritten in the following way.

Theorem 2.2. For $2 g-2+n>0$, we have

$$
K \widehat{\mathcal{F}}_{g, n}=\frac{1}{2}\left(\mathcal{D} \mathcal{D} \widehat{\mathcal{F}}_{g-1, n}+\sum_{\substack{g_{1}+g_{2}=g, n 1+n 2}} \mathcal{D} \widehat{\mathcal{F}}_{g_{1}, n_{1}} \mathcal{D} \widehat{\mathcal{F}}_{g_{2}, n_{2}}\right) .
$$

In particular, by taking $n=0$ we get a recursion relation for the abstract free energy:

$$
K \widehat{\mathcal{F}}_{g}=\frac{1}{2}\left(\mathcal{D} \partial \widehat{\mathcal{F}}_{g-1}+\sum_{r=1}^{g-1} \partial \widehat{\mathcal{F}}_{r} \partial \widehat{\mathcal{F}}_{g-r}\right) .
$$

\section{A generalization to labelled graphs}

In this section, we generalize the construction of section 2 to the case where all the halfedges of graphs may have labels.

\subsection{Diagrammatics for labelled graphs}

Fix a positive integer $N$, for a stable graph $\Gamma \in \mathcal{G}_{g, n}$ we label some indices on the edges as follows. For an external edge, we label an index in $\{1,2, \cdots, N\}$; and for an internal edge, we label an index in $\{1,2, \cdots, N\}$ at each of its end points. In other words, we label each half edge incident at a vertex. 
As generalizations of the edge-cutting operator $K$ defined in section 2.2, we define the edge-cutting operators $K_{i j}$ as the operator that cuts off an internal edges with two labels $i$ and $j$, then sum over all such internal edges.

Example 3.1. Take $N=2$, then we have the following examples:

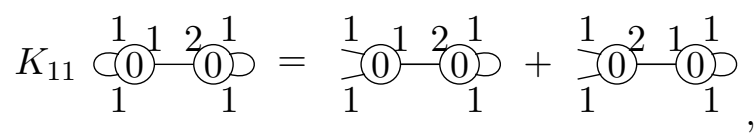

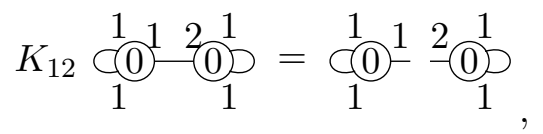

$$
\begin{aligned}
& K_{22} \underset{1}{1}-\frac{2}{1}-(0)=0
\end{aligned}
$$

Similarly, we also have operators $\partial_{i}$ and $\gamma_{i}$ generalizing the operators $\partial$ and $\gamma$ in section 2.2 respectively. The operator $\partial_{i}$ has two parts, one is to add an external edge labelled by $i$, and the other is to break up an internal edge and attach a 3-pointed Riemann sphere such that the new external edge has label $i$. The operator $\gamma_{i}$ is to attach a 3-pointed Riemann sphere to an external edge $e$, and move the label of $e$ to be the label of one of the new external edge, while the other new external edge has label $i$. The two labels of the new internal edge can be chosen arbitrarily.

Example 3.2. For $N=2$, here are some examples of the operator $\partial_{i}$.

$$
\begin{aligned}
& \partial_{1}(1)^{2}=(1) \underset{1}{2}
\end{aligned}
$$

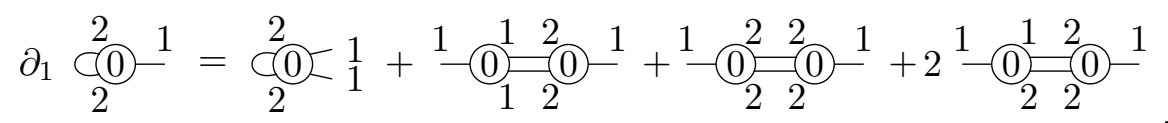

Example 3.3. For $N=2$, here are some examples of the operator $\gamma_{i}$.

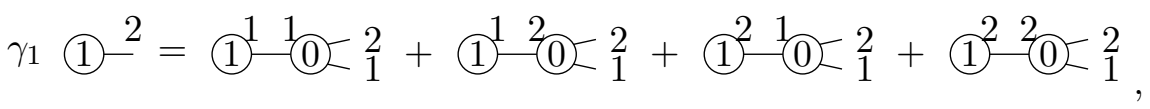

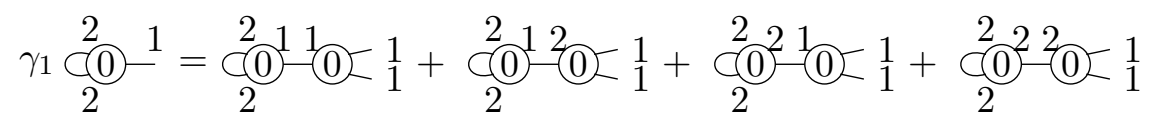

Now let $\mathcal{G}_{g, n}(N)$ be the set of all labelled stable graphs of genus $g$ with $n$ external edges and $N$ possible indices, and $\mathcal{G}_{g, n}^{c}(N)$ be the set of connected ones. In the labelled case we also define the abstract free energy in the same way.

Definition 3.1. For $g \geq 2$, the abstract free energy $\widehat{\mathcal{F}}_{g}$ is defined by:

$$
\widehat{\mathcal{F}}_{g}=\sum_{\Gamma \in \mathcal{G}_{g, 0}^{c}(N)} \frac{1}{|\operatorname{Aut}(\Gamma)|} \Gamma .
$$


For example, take $N=2$, then we have

$$
\begin{aligned}
& \widehat{\mathcal{F}}_{2}=(2)+\frac{1}{2}(1)^{1}{ }^{1}(1)+\frac{1}{2}(1)^{2}-2(1)+(1)^{1}{ }^{2}(1) \\
& +\frac{1}{2} \underset{1}{C(1)}+\frac{1}{2} \underset{2}{\stackrel{2}{C}(1)}+\underset{2}{\stackrel{1}{C}(1)}+\cdots
\end{aligned}
$$

The complete expressions for $\widehat{\mathcal{F}}_{2}$ can be obtained by adding all possible labels on the graphs of $\widehat{\mathcal{F}}_{2}$ for the $N=1$ case and modifying the number of automorphisms.

In this case we also have the notions of abstract $n$-point functions. Denote by $\mathcal{G}_{g ; l_{1}, \cdots, l_{N}}^{c}(N) \subset \mathcal{G}_{g, n}^{c}(N)$ the subset consisting of all connected labelled stable graphs of genus $g$, with $l_{j}$ external edges labelled by $j$ for every $j \in\{1, \cdots, N\}$ (we have $\left.l_{1}+\cdots+l_{N}=n\right)$. Then we define the abstract $n$-point function $\widehat{\mathcal{F}}_{g ; l_{1}, \cdots, l_{N}}$ to be the linear combination

$$
\widehat{\mathcal{F}}_{g ; l_{1}, \cdots, l_{N}}:=\sum_{\Gamma \in \mathcal{G}_{g ; l_{1}, \cdots, l_{N}}^{c}(N)} \frac{1}{|\operatorname{Aut}(\Gamma)|} \Gamma,
$$

for $2 g-2+\sum l_{j}>0$

\subsection{A recursion relation for the abstract free energy}

In the labelled case, we also have a recursion relation generalizing (2.37). Here we define $\mathcal{D}_{i}=\partial_{i}+\gamma_{i}$.

Theorem 3.1. For $g \geq 2$, we have

$$
K_{i j} \widehat{\mathcal{F}}_{g}=\mathcal{D}_{i} \partial_{j} \widehat{\mathcal{F}}_{g-1}+\sum_{r=1}^{g-1} \partial_{i} \widehat{\mathcal{F}}_{r} \partial_{j} \widehat{\mathcal{F}}_{g-r}
$$

for $i \neq j$, and

$$
K_{i i} \widehat{\mathcal{F}}_{g}=\frac{1}{2}\left(\mathcal{D}_{i} \partial_{i} \widehat{\mathcal{F}}_{g-1}+\sum_{r=1}^{g-1} \partial_{i} \widehat{\mathcal{F}}_{r} \partial_{i} \widehat{\mathcal{F}}_{g-r}\right) .
$$

The proof is an easy modification of the proof of Theorem 2.2 and is omitted. For the case of $g=2$ we use the following convention

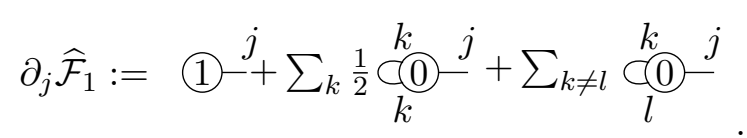

The recursion relations for the abstract $n$-point functions can also be generalized to the case of labelled graphs. The generalizations of Lemma 2.1 and Theorem 2.2 are the following:

Lemma 3.1. For $2 g-2+\sum l_{j}>0$, we have

$$
\mathcal{D}_{j} \widehat{\mathcal{F}}_{g ; l_{1}, \cdots, l_{N}}=\left(l_{j}+1\right) \cdot \widehat{\mathcal{F}}_{g ; l_{1}, \cdots, l_{j}+1, \cdots, l_{N}} .
$$


Theorem 3.2. For $2 g-2+\sum l_{j}>0$, we have

$$
\begin{aligned}
& K_{i j} \widehat{\mathcal{F}}_{g ; l_{1}, \cdots, l_{N}}=\mathcal{D}_{i} \mathcal{D}_{j} \widehat{\mathcal{F}}_{g-1 ; l_{1}, \cdots, l_{N}}+\sum_{\substack{g_{1}+g_{2}=g \\
p_{k}+q_{k}=l_{k}}} \mathcal{D}_{i} \widehat{\mathcal{F}}_{g_{1} ; p_{1}, \cdots, p_{N}} \mathcal{D}_{j} \widehat{\mathcal{F}}_{g_{2} ; q_{1}, \cdots, q_{N}}, \quad i \neq j ; \\
& K_{i i} \widehat{\mathcal{F}}_{g ; l_{1}, \cdots, l_{N}}=\frac{1}{2}\left(\mathcal{D}_{i} \mathcal{D}_{i} \widehat{\mathcal{F}}_{g-1 ; l_{1}, \cdots, l_{N}}+\sum_{\substack{g_{1}+g_{2}=g \\
p_{k}+q_{k}=l_{k}}} \mathcal{D}_{i} \widehat{\mathcal{F}}_{g_{1} ; p_{1}, \cdots, p_{N}} \mathcal{D}_{i} \widehat{\mathcal{F}}_{g_{2} ; q_{1}, \cdots, q_{N}}\right) .
\end{aligned}
$$

In particular, for $l_{1}=\cdots=l_{N}=0$ these recover the recursion relations in Theorem 3.1. Here we use the convention

$$
\begin{aligned}
& \partial_{j} \widehat{\mathcal{F}}_{1 ; l_{1}, \cdots, l_{N}}:=\widehat{\mathcal{F}}_{1 ; l_{1}, \cdots, l_{j}+1, \cdots, l_{N}}, \quad \text { for } \quad l_{1}=\cdots=l_{N}=0 ; \\
& \mathcal{D}_{j} \widehat{\mathcal{F}}_{0 ; l_{1}, \cdots, l_{N}}=\left(l_{j}+1\right) \widehat{\mathcal{F}}_{0 ; l_{1}, \cdots, l_{j}+1, \cdots, l_{N}}, \quad \text { for } \quad l_{1}+\cdots+l_{N}=2 ; \\
& \mathcal{D}_{i} \mathcal{D}_{j} \widehat{\mathcal{F}}_{0 ; l_{1}, \cdots, l_{N}}:=\left(l_{j}+1\right) \mathcal{D}_{i} \widehat{\mathcal{F}}_{0 ; l_{1}, \cdots, l_{j}+1, \cdots, l_{N}}, \quad \text { for } \quad l_{1}+\cdots+l_{N}=1 .
\end{aligned}
$$

\section{Realization of the abstract quantum field theory}

In this section we formulate a Feynman rule for the diagrammatics discussed in the previous sections, in such way we are able to define a new free energy $\widehat{F}$ from a given holomorphic free energy $F$ and a choice of propagator $\kappa$. We also represent this new field theory as formal Gaussian integrals. This construction realizes the abstract free energy and its quadratic recursion relations discussed in preceding sections.

\subsection{Realization of the abstract QFT by Feynman rules}

In this subsection we will present a construction to realize the abstract quantum field theory defined in section 2.3 and section 3.1 by Feynman rules that assign the contribution of a vertex and the contribution of edge:

$$
\Gamma \mapsto \omega_{\Gamma}=\prod_{v \in V(\Gamma)} \omega_{v} \cdot \prod_{e \in E(\Gamma)} \omega_{e} .
$$

For $\omega_{v}$, we need to fix a sequence of holomorphic functions $F_{g}$ in $t_{1}, \ldots, t_{N}$ as input; for $\omega_{e}$, we need fix a nondegenerate symmetric matrix $\kappa=\left(\kappa_{i j}\right)_{1 \leq i, j \leq N}$ of functions in $t_{1}, \ldots, t_{N}$ as propagators.

For a given positive integer $N$, let $t_{1}, t_{2}, \cdots, t_{N}$ be the coordinates on an $N$-dimensional vector space, and $\kappa=\left(\kappa_{i j}\right)$ be a nondegenerate symmetric matrix of size $N \times N$. Moreover, we fix a sequence of holomorphic function $F_{g}\left(t_{1}, \cdots, t_{N}\right)$ for $g \geq 0$. Let

$$
F(t ; \lambda)=\sum_{g=0}^{\infty} \lambda^{2 g-2} F_{g}(t) .
$$

Now for a labelled stable graph $\Gamma \in \mathcal{G}_{g, 0}^{c}(N)$, we associate a polynomial $\omega_{\Gamma}$ as follows. Let $V(\Gamma), E^{\text {ext }}(\Gamma)$, and $E(\Gamma)$ be the sets of vertices, external half edges, and internal edges of $\Gamma$ respectively. For each $e \in E^{\operatorname{ext}}(\Gamma)$, write $l(e)$ as the label on the external $e$; and for 
each $e \in E(\Gamma)$, write $l_{1}(e)$ and $l_{2}(e)$ as the two labels on $e$ (the order is not important). For each $v \in V(\Gamma)$, write $g(v)$ as the genus associated to $v$, and $\operatorname{val}_{i}(v)$ as the number of half-edges labelled by $i$ incident at $v$, then $\sum_{i} \operatorname{val}_{i}(v)$ is the valence of $v$. Then $\omega_{\Gamma}$ is defined by

$$
\omega_{\Gamma}=\prod_{v \in V(\Gamma)} F_{g(v)}^{\left(\operatorname{val}_{1}(v), \cdots, \operatorname{val}_{N}(v)\right)}(t) \prod_{e \in E(\Gamma)} \kappa_{l_{1}(e) l_{2}(e)},
$$

where $F_{g(v)}^{\left(\operatorname{val}_{1}(v), \cdots, \operatorname{val}_{N}(v)\right)}(t):=\left(\frac{\partial}{\partial t_{1}}\right)^{\operatorname{val}_{1}(v)} \cdots\left(\frac{\partial}{\partial t_{N}}\right)^{\operatorname{val}_{N}(v)} F_{g(v)}(t)$. For $N=1$, this expression simplifies to

$$
\omega_{\Gamma}=\left(\prod_{v \in V(\Gamma)} F_{g(v)}^{(\operatorname{val}(v))}(t)\right) \kappa^{|E(\Gamma)|} .
$$

Definition 4.1. For $g \geq 2$ we define the free energy to be

$$
\widehat{F}_{g}=\sum_{\Gamma \in \mathcal{G}_{g, 0}^{c}(N)} \frac{1}{|\operatorname{Aut}(\Gamma)|} \omega_{\Gamma} .
$$

In particular, the degree of $\kappa$ in the expression of $\widehat{F}_{g}$ is $3 g-3$.

Example 4.1. For $N=1$, we have

$$
\widehat{F}_{2}=F_{2}+\kappa\left[\frac{1}{2} F_{1}^{\prime \prime}+\frac{1}{2}\left(F_{1}^{\prime}\right)^{2}\right]+\kappa^{2}\left(\frac{1}{8} F_{0}^{(4)}+\frac{1}{2} F_{1}^{\prime} F_{0}^{\prime \prime \prime}\right)+\frac{5}{24} \kappa^{3}\left(F_{0}^{\prime \prime \prime}\right)^{2} .
$$

And for $N=2$, we have

$$
\begin{aligned}
\widehat{F}_{2}= & F_{2}+\left(\frac{1}{2} F_{1}^{(2,0)}+\frac{1}{2}\left(F_{1}^{(1,0)}\right)^{2}\right) \kappa_{11}+\left(\frac{1}{2} F_{1}^{(0,2)}+\frac{1}{2}\left(F_{1}^{(0,1)}\right)^{2}\right) \kappa_{22} \\
& +\left(\frac{1}{8} F_{0}^{(4,0)}+\frac{1}{2} F_{1}^{(1,0)} F_{0}^{(3,0)}\right) \kappa_{11}^{2}+\left(\frac{1}{8} F_{0}^{(0,4)}+\frac{1}{2} F_{1}^{(0,1)} F_{0}^{(0,3)}\right) \kappa_{22}^{2} \\
& +\left(F_{1}^{(1,1)}+F_{1}^{(1,0)} F_{1}^{(0,1)}\right) \kappa_{12}+\left(\frac{1}{2} F_{0}^{(2,2)}+F_{1}^{(1,0)} F_{0}^{(1,2)}+F_{1}^{(0,1)} F_{0}^{(2,1)}\right) \kappa_{12}^{2} \\
& +\left(\frac{1}{4} F_{0}^{(2,2)}+\frac{1}{2} F_{1}^{(1,0)} F_{0}^{(1,2)}+\frac{1}{2} F_{1}^{(0,1)} F_{0}^{(2,1)}\right) \kappa_{11} \kappa_{22} \\
& +\left(\frac{1}{2} F_{0}^{(3,1)}+\frac{3}{2} F_{1}^{(1,0)} F_{0}^{(2,1)}+\frac{1}{2} F_{1}^{(0,1)} F_{0}^{(3,0)}\right) \kappa_{11} \kappa_{12} \\
& +\left(\frac{1}{2} F_{0}^{(1,3)}+\frac{3}{2} F_{1}^{(0,1)} F_{0}^{(1,2)}+\frac{1}{2} F_{1}^{(1,0)} F_{0}^{(0,3)}\right) \kappa_{22} \kappa_{12} \\
& +\frac{5}{24}\left(F_{0}^{(3,0)}\right)^{2} \kappa_{11}^{3}++\frac{5}{24}\left(F_{0}^{(0,3)}\right)^{2} \kappa_{22}^{3}+\left(\frac{1}{6} F_{0}^{(3,0)} F_{0}^{(0,3)}+\frac{3}{2} F_{0}^{(2,1)} F_{0}^{(1,2)}\right) \kappa_{12}^{3} \\
& +\left(\frac{3}{8}\left(F_{0}^{(2,1)}\right)^{2}+\frac{1}{4} F_{0}^{(3,0)} F_{0}^{(1,2)}\right) \kappa_{11}^{2} \kappa_{22} \\
& +\left(\frac{3}{8}\left(F_{0}^{(1,2)}\right)^{2}+\frac{1}{4} F_{0}^{(0,3)} F_{0}^{(2,1)}\right) \kappa_{22}^{2} \kappa_{11} \\
& +\left(\frac{3}{2}\left(F_{0}^{(2,1)}\right)^{2}+F_{0}^{(3,0)} F_{0}^{(1,2)}\right) \kappa_{11} \kappa_{12}^{2}+\left(\frac{3}{2}\left(F_{0}^{(1,2)}\right)^{2}+F_{0}^{(0,3)} F_{0}^{(2,1)}\right) \kappa_{22} \kappa_{12}^{2} \\
& +\frac{5}{4} F_{0}^{(3,0)} F_{0}^{(2,1)} \kappa_{11}^{2} \kappa_{12}+\frac{5}{4} F_{0}^{(0,3)} F_{0}^{(1,2)} \kappa_{22}^{2} \kappa_{12} \\
& +\left(\frac{1}{4} F_{0}^{(3,0)} F_{0}^{(0,3)}+\frac{9}{4} F_{0}^{(2,1)} F_{0}^{(1,2)}\right) \kappa_{11} \kappa_{22} \kappa_{12} .
\end{aligned}
$$




\subsection{Representation by formal Gaussian integrals}

In fact, the free energy $\widehat{F}_{g}$ defined in last subsection has a representation using formal Gaussian integrals. Consider the following partition function

$$
\begin{gathered}
Z(t, \kappa)=\int \exp \left\{F(\eta)-\lambda^{-2}\left[\frac{\partial F_{0}(t)}{\partial t}(\eta-t)+\frac{1}{2}(\eta-t)^{T} \frac{\partial^{2} F_{0}(t)}{\partial t^{2}}(\eta-t)\right.\right. \\
\left.\left.+\frac{1}{2}(\eta-t)^{T} \kappa^{-1}(\eta-t)\right]\right\} d \eta
\end{gathered}
$$

where the integral is over $\eta \in \mathbb{R}^{N}$. Now do the Taylor expansion of $F(\eta)$, we get

$$
\begin{aligned}
& Z(t, \kappa)=Z(t) \int \exp \left[\sum_{2 g-2+\sum l^{(i)}>0} \frac{\lambda^{2 g-2}}{\prod_{i=1}^{N} l^{(i)} !} F_{g}^{\left(l^{(1)}, \cdots, l^{(N)}\right)}(t) \prod_{j=1}^{N}\left(\eta_{i}-t_{i}\right)^{l^{(i)}}\right. \\
& \left.-\frac{\lambda^{-2}}{2}(\eta-t)^{T} \kappa^{-1}(\eta-t)\right] d \eta \\
& =Z(t) \sum_{k \geq 0} \sum_{2 g_{j}-2+\sum_{i} l_{j}^{(i)}>0} \frac{1}{k !} \frac{\lambda^{2 \sum_{k} g_{k}-2 k}}{\prod_{1 \leq j \leq k} \prod_{i} l_{j}^{(i)} !} \prod_{j=1}^{k} F_{g_{j}}^{\left(l_{j}^{(1)}, \cdots, l_{j}^{(N)}\right)} \\
& \times \int \prod_{i=1}^{N}\left(\eta_{i}-t_{i}\right)^{\sum_{j=1}^{k} l_{j}^{(i)}} \cdot \exp \left[-\frac{\lambda^{-2}}{2}(\eta-t)^{T} \kappa^{-1}(\eta-t)\right] d \eta,
\end{aligned}
$$

where $Z(t)=\exp (F(t))$ is the holomorphic partition function. Then evaluate the Gaussian integral on the right-hand side of the last equality, we have

$$
\begin{aligned}
& \int \prod_{i=1}^{N}\left(\eta_{i}-t_{i}\right)^{\sum_{j=1}^{k} l_{j}^{(i)}} \cdot \exp \left[-\frac{\lambda^{-2}}{2}(\eta-t)^{T} \kappa^{-1}(\eta-t)\right] d \eta \\
= & \left(\frac{(2 \pi)^{n}}{\operatorname{det}\left(\lambda^{-2} \kappa^{-1}\right)}\right)^{\frac{1}{2}} \cdot \frac{1}{2^{l} \cdot l !} \sum_{\sigma \in S_{2 l}} \prod_{j=1}^{l}\left(\lambda^{2} \kappa_{a_{\sigma(2 j-1)} a_{\sigma(2 j)}}\right),
\end{aligned}
$$

for $\sum_{i, j} l_{j}^{(i)}=2 l$ even, and equals zero if $\sum_{i, j} l_{j}^{(i)}$ is odd, where the sequence $\left(a_{1}, \cdots, a_{2 l}\right)$ consists of indices in $\{1, \cdots, N\}$ with the number of $i$ equals $\sum_{j} l_{j}^{(i)}$.

Let the normalized partition function be

$$
\widetilde{Z}(t, \kappa)=Z(t, \kappa) /\left(2 \pi \lambda^{2}\right)^{\frac{N}{2}},
$$

then this relates to our free energy (4.5) in the following way.

Theorem 4.1. We have

$$
\widetilde{Z}(t, \kappa)=\exp \left(\sum_{g=0}^{\infty} \lambda^{2 g-2} \widehat{F}_{g}(t)\right),
$$

where $\widehat{F}_{0}$ and $\widehat{F}_{1}$ are defined by

$$
\widehat{F}_{0}=F_{0}, \quad \widehat{F}_{1}=F_{1}+\frac{1}{2} \log (\operatorname{det}(\kappa)) .
$$


Proof. From (4.9) and (4.10), the coefficient of $\lambda^{n}$ in $\widetilde{Z}(t, \kappa) /(Z(t) \sqrt{\operatorname{det}(\kappa)})$ is

$$
\sum_{2 \sum g_{j}-2 k+2 l=n} \frac{1}{k !} \cdot \frac{1}{2^{l} \cdot l !} \prod_{j=1}^{k} \frac{F_{g_{j}}^{\left(l_{j}^{(1)}, \cdots, l_{j}^{(N)}\right)}}{l_{j}^{(1)} ! \cdots l_{j}^{(N)} !} \cdot \sum_{\sigma \in S_{2 l}} \prod_{j=1}^{l} \kappa_{a_{\sigma(2 j-1)} a_{\sigma(2 j)}} .
$$

This corresponds to the summation of $\omega_{\Gamma}$ over all possible ways to glue some stable vertices of types $\left\{F_{g_{j}}^{\left(l_{j}^{(1)}, \cdots, l_{j}^{(N)}\right)}\right\}_{1 \leq j \leq l}$ together to obtain a stable graph $\Gamma$ (not necessarily connected), with coefficients $\frac{1}{|\operatorname{Aut}(\Gamma)|}$. Then since

$$
l=|E(\Gamma)|, \quad k=|V(\Gamma)|,
$$

we have

$$
g(\Gamma)=1+l-k+\sum_{j=1}^{k} g_{j}
$$

Thus

$$
\widetilde{Z}(t, \kappa)=\sum_{\Gamma \in \mathcal{G}_{g, 0}} \frac{\lambda^{2 g-2}}{|\operatorname{Aut}(\Gamma)|} \omega_{\Gamma}
$$

and

$$
\log (\widetilde{Z})=\sum_{\Gamma \in \mathcal{G}_{g, 0}^{c}} \frac{\lambda^{2 g-2}}{|\operatorname{Aut}(\Gamma)|} \omega_{\Gamma}=\sum_{g=0}^{\infty} \lambda^{2 g-2} \widehat{F}_{g}
$$

Example 4.2. For the case of $N=1$, the partition function is

$$
\begin{aligned}
\tilde{Z}(t, \kappa)= & \exp \left(\sum_{g=0}^{\infty} \lambda^{2 g-2} \widehat{F}_{g}(t)\right) \\
= & Z(t) \sum_{k \geq 0} \sum_{2 g_{i}-2+l_{i}>0} \sum_{l} \frac{1}{k !} \frac{\lambda^{2\left(g_{1}+\cdots+g_{k}\right)-2 k+2 l}}{l_{1} ! \cdots l_{k} !} \\
& \times(2 l-1) ! ! \cdot F_{g_{1}}^{\left(l_{1}\right)} \cdots F_{g_{k}}^{\left(l_{k}\right)} \cdot \kappa^{l} \cdot \delta_{l_{1}+\cdots+l_{k}-2 l, 0} .
\end{aligned}
$$

As mentioned in the Introduction, the formal Gaussian integral (4.8) appeared in earlier work on holomorphic anomaly equations $[15,17,19]$, where the propagator $\kappa$ was chosen to be the Zamolodchikov metric which is non-holomorphic. Later in this paper we will see that the propagator $\kappa$ admits different choices, which give us different theories in mathematical physics. In particular, by taking suitable holomorphic propagators we will see that partition functions for some well-known quantum spectral curves can be constructed in our formalism.

\subsection{Realization of the operators on stable graphs}

Now we discuss the realization of the operators $K, \partial$ and $\gamma$ defined in section 2.2. 
The operator $K$ is realized by the differential operator $\partial_{\kappa}$. In fact, by (4.4),

$$
\begin{aligned}
\partial_{\kappa} \omega_{\Gamma} & =\partial_{\kappa}\left[\left(\prod_{v \in V(\Gamma)} F_{g(v)}^{(\operatorname{val}(v))}(t)\right) \kappa^{|E(\Gamma)|}\right] \\
& =|E(\Gamma)| \cdot\left(\prod_{v \in V(\Gamma)} F_{g(v)}^{(\operatorname{val}(v))}(t)\right) \kappa^{|E(\Gamma)|-1} \\
& =\sum_{\Gamma^{\prime}} \omega_{\Gamma^{\prime}}=\omega_{K \Gamma},
\end{aligned}
$$

where the summation on the right-hand side of the second equality is over all graphs $\Gamma^{\prime}$ obtained from $\Gamma$ by cutting an edge.

The operator $\gamma$ is realized by the operator of multiplication by $\left|E^{\operatorname{ext}}(\Gamma)\right| \cdot \kappa F_{0}^{\prime \prime \prime}$ on $\omega_{\Gamma}$. In fact, the effect of $\gamma$ is to change each external edge incident at $v$ of a stable graph $\Gamma$ to an internal edge joining the vertex $v$ to an additional trivalent vertex of genus 0 . Let $\Gamma^{\prime}$ be one of stable graphs obtained from $\Gamma$ in this way, then by the Feynman rule,

$$
\omega_{\Gamma^{\prime}}=\kappa F_{0}^{\prime \prime \prime} \cdot \omega_{\Gamma}
$$

Therefore,

$$
\omega_{\gamma \Gamma}=\sum_{\Gamma^{\prime}} \omega_{\Gamma^{\prime}}=\left|E^{e x t}(\Gamma)\right| \cdot \kappa F_{0}^{\prime \prime \prime} \cdot \omega_{\Gamma}
$$

The operator $\partial$ is realized by the differential operator $\partial_{t}$. In fact, $\omega_{\Gamma}$ is a product of contributions from the vertices and the contributions from the internal edges. Hence $\partial_{t} \omega_{\Gamma}$ is a summation of the contributions of the actions on each vertices and the contributions on each internal edges. In fact by (4.4),

$$
\begin{aligned}
\partial_{t} \omega_{\Gamma}= & \partial_{t}\left[\left(\prod_{v \in V(\Gamma)} F_{g(v)}^{(\operatorname{val}(v))}(t)\right) \kappa^{|E(\Gamma)|}\right] \\
= & \left.\sum_{v \in V(\Gamma)} F_{g(v)}^{(\operatorname{val}(v)+1)}(t) \cdot \prod_{v^{\prime} \in V(\Gamma)-\{v\}} F_{g\left(v^{\prime}\right)}^{\left(\operatorname{val}\left(v^{\prime}\right)\right)}(t)\right) \cdot \kappa^{|E(\Gamma)|} \\
& \left.+\prod_{v \in V(\Gamma)} F_{g(v)}^{(\operatorname{val}(v))}(t)\right) \cdot|E(\Gamma)| \kappa^{|E(\Gamma)|-1} \cdot \partial_{t} \kappa .
\end{aligned}
$$

On the other hand,

$$
\partial \Gamma=\sum_{v \in V(\Gamma)} \Gamma^{v}+\sum_{e \in E(\Gamma)} \Gamma^{e}
$$

where $\Gamma^{v}$ is obtained from $\Gamma$ by attaching an additional half-edge at a vertex $v \in V(\Gamma)$, and $\Gamma^{e}$ is obtained from $\Gamma$ by changing an internal edge $e \in E(\Gamma)$ to two internal edges incident at an addition trivalent vertex $w$ of genus zero. Therefore, by the Feynman rule 
we get:

$$
\begin{aligned}
\omega_{\partial \Gamma}= & \sum_{v \in V(\Gamma)} \omega_{\Gamma^{v}}+\sum_{e \in E(\Gamma)} \omega_{\Gamma^{e}} \\
= & \sum_{v \in V(\Gamma)} F_{g(v)}^{(\operatorname{val}(v)+1)}(t) \cdot \prod_{v^{\prime} \in V(\Gamma)-\{v\}} F_{g\left(v^{\prime}\right)}^{\left(\operatorname{val}\left(v^{\prime}\right)\right)}(t) \cdot \kappa^{|E(\Gamma)|} \\
& +\sum_{e \in E(\Gamma)} \prod_{v \in V(\Gamma)} F_{g(v)}^{(\operatorname{val}(v))}(t) \cdot \kappa^{|E(\Gamma)|+1} \cdot F_{0}^{\prime \prime \prime}(t) .
\end{aligned}
$$

Therefore, in order to have $\omega_{\partial \Gamma}=\partial_{t} \omega_{\Gamma}$, we require the propagator $\kappa$ to be a function of $t$ which satisfies

$$
\frac{\partial \kappa}{\partial t}=\kappa^{2} \cdot F_{0}^{\prime \prime \prime}(t)
$$

here $\kappa$ needs not to be holomorphic. A general solution to this equation is

$$
\kappa(t)=\frac{1}{C-F_{0}^{\prime \prime}(t)},
$$

where $C$ may be either a constant or an anti-holomorphic function of $t$.

\subsection{Realization of the recursion relations}

By applying Feynman rules to the quadratic recursion relations in Theorem 2.2 and using the realizations of the operators on graphs discussion in the preceding subsection, we obtain the recursion relations for $\widehat{F}_{g}$ immediately.

Theorem 4.2. When the propagator $\kappa$ satisfies the condition (4.18), the free energy $\widehat{F}_{g}$ for $g \geq 2$ satisfies the equations

$$
\partial_{\kappa} \widehat{F}_{g}=\frac{1}{2}\left(D_{t} \partial_{t} \widehat{F}_{g-1}+\sum_{r=1}^{g-1} \partial_{t} \widehat{F}_{r} \partial_{t} \widehat{F}_{g-r}\right),
$$

where $D_{t}=\partial_{t}+\kappa F_{0}^{\prime \prime \prime}$ is the covariant derivative.

Example 4.3. For the expression (4.6) of $\widehat{F}_{2}$, we have

$$
\begin{aligned}
\partial_{\kappa} \widehat{F}_{2}= & \frac{1}{2} F_{1}^{\prime \prime}+\frac{1}{2}\left(F_{1}^{\prime}\right)^{2}+\kappa\left(\frac{1}{4} F_{0}^{(4)}+F_{1}^{\prime} F_{0}^{\prime \prime \prime}\right)+\frac{5}{8} \kappa^{2}\left(F_{0}^{\prime \prime \prime}\right)^{2}, \\
\partial_{t} \widehat{F}_{2}= & F_{2}^{\prime}+\kappa\left[\frac{1}{2} F_{1}^{\prime \prime \prime}+F_{1}^{\prime} F_{1}^{\prime \prime}\right]+\kappa^{2}\left(\frac{1}{8} F_{0}^{(5)}+\frac{1}{2} F_{1}^{\prime} F_{0}^{(4)}+\frac{1}{2}\left(F_{1}^{\prime}\right)^{2} F_{0}^{\prime \prime \prime}+F_{1}^{\prime \prime} F_{0}^{\prime \prime \prime}\right) \\
& +\kappa^{3}\left[F_{1}^{\prime}\left(F_{0}^{\prime \prime \prime}\right)^{2}+\frac{2}{3} F_{0}^{\prime \prime \prime} F_{0}^{(4)}\right]+\frac{5}{8} \kappa^{4}\left(F_{0}^{\prime \prime \prime}\right)^{3} .
\end{aligned}
$$

Here in the second equality we have used (4.18).

Example 4.4. Direct computation gives

$$
D_{t} \partial_{t} \widehat{F}_{1}=F_{1}^{\prime \prime}+\kappa\left(\frac{1}{2} F_{0}^{(4)}+F_{1}^{\prime} F_{0}^{\prime \prime \prime}\right)+\kappa^{2}\left(F_{0}^{\prime \prime \prime}\right)^{2},
$$

one may check that

$$
\partial_{\kappa} \widehat{F}_{2}=\frac{1}{2}\left(D_{t} \partial_{t} \widehat{F}_{1}+\left(\partial_{t} \widehat{F}_{1}\right)^{2}\right)
$$




\subsection{The general case}

Now we return to the general case for an arbitrary dimension $N$. Similar to $N=1$ case, the operators $K_{i j}$ and $\partial_{i}$ defined in section 3 are realized by $\partial_{\kappa_{i j}}$ and $\partial_{t_{i}}$ respectively. To realize the operator $\partial_{i}$ as the differential operator $\partial_{t_{i}}$, we need to impose some conditions on the propagators $\kappa_{i j}$ analogous to (4.18):

$$
\frac{\partial \kappa_{j k}}{\partial t_{i}}=\sum_{l, m} \kappa_{j l} \kappa_{k m} \frac{\partial^{3} F_{0}}{\partial t_{i} \partial t_{l} \partial t_{m}}
$$

or in matrix form:

$$
\frac{\partial \kappa}{\partial t_{k}}=\kappa \frac{\partial H\left(F_{0}\right)}{\partial t_{k}} \kappa
$$

where $H\left(F_{0}\right)=\left(\frac{\partial^{2} F_{0}}{\partial t_{i} \partial t_{j}}\right)$ is the Hessian of $F_{0}$. A general solution is given by

$$
\kappa(t)=\left(C-H\left(F_{0}\right)\right)^{-1}
$$

where $C$ is a symmetric anti-holomorphic or constant matrix such that $\left(C-H\left(F_{0}\right)\right)$ is invertible.

The realization of the operator $\mathcal{D}_{i}=\partial_{i}+\gamma_{i}$ is more subtle. For our purpose, it suffices to define the operator $D_{t_{i}} \partial_{t_{j}}$ as a realization of the operator $\mathcal{D}_{i} \partial_{j}$ as follows.

Definition 4.2. For $\Gamma \in \mathcal{G}_{g, 0}^{c}$, we define

$$
\begin{aligned}
D_{t_{i}} \partial_{t_{j}} \omega_{\Gamma} & =\omega_{D_{i} \partial_{j} \Gamma} \\
& =\frac{\partial^{2}}{\partial t_{i} \partial t_{j}} \omega_{\Gamma}+\sum_{l, m=1}^{N} \frac{\partial}{\partial t_{l}} \omega_{\Gamma} \cdot \kappa_{l m}(t) \frac{\partial^{3} F_{0}}{\partial t_{m} \partial t_{i} \partial t_{j}},
\end{aligned}
$$

where

$$
\omega_{\Gamma}=\prod_{v \in V(\Gamma)} F_{g(v)}^{\left(\operatorname{val}_{1}(v), \cdots, \operatorname{val}_{N}(v)\right)}(t) \cdot \prod_{e \in E(\Gamma)} \kappa_{l_{1}(e) l_{2}(e)}(t, \bar{t}) .
$$

In particular, we see that $D_{t_{i}} \partial_{t_{j}} \omega_{\Gamma}=D_{t_{j}} \partial_{t_{i}} \omega_{\Gamma}$.

Then by applying the Feynman rules, Theorem 3.1 gives us

Theorem 4.3. When the condition (4.21) is satisfied, for $g \geq 2$, we have the equations

$$
\partial_{\kappa_{i j}} \widehat{F}_{g}=D_{t_{i}} \partial_{t_{j}} \widehat{F}_{g-1}+\sum_{r=1}^{g-1} \partial_{t_{i}} \widehat{F}_{r} \partial_{t_{j}} \widehat{F}_{g-r}
$$

for $i \neq j$, and

$$
\partial_{\kappa_{i i}} \widehat{F}_{g}=\frac{1}{2}\left(D_{t_{i}} \partial_{t_{i}} \widehat{F}_{g-1}+\sum_{r=1}^{g-1} \partial_{t_{i}} \widehat{F}_{r} \partial_{t_{i}} \widehat{F}_{g-r}\right)
$$

for the free energy $\widehat{F}_{g}$ defined by (4.5). 


\section{The holomorphic anomaly equation}

In the rest of this paper we will present some examples of our formalism developed above. In this section we will focus on the case of holomorphic anomaly equations.

As we have mentioned in the Introduction, the holomorphic anomaly equation was introduced by Bershadsky et al. in physics literatures [2,3] in order to compute the GromovWitten invariants of the quintic Calabi-Yau threefold. They developed a method to solve the non-holomorphic free energies $\mathcal{F}_{g}(t, \bar{t})$ recursively using the holomorphic anomaly equations

$$
\bar{\partial}_{\bar{i}} \mathcal{F}_{g}=\frac{1}{2} \bar{C}_{\bar{i} \bar{j} \bar{k}} e^{2 K} G^{j \bar{j}} G^{k \bar{k}}\left(D_{j} D_{k} \mathcal{F}_{g-1}+\sum_{r=1}^{g-1} D_{j} \mathcal{F}_{r} D_{k} \mathcal{F}_{g-r}\right), \quad g \geq 2,
$$

and by formally 'taking a limit'

$$
F_{g}(t)=\lim _{\bar{t} \rightarrow \infty} \mathcal{F}_{g}(t, \bar{t})
$$

they got the generating series of Gromov-Witten invariants $F(t)$ of the threefold on the small phase space. The non-holomorphic free energies $\mathcal{F}_{g}(t, \bar{t})$ are supposed to have modularity while the holomorphic free energies $F_{g}(t)$ does not, and this has been exploited to use the theory of modular forms. Since the ring of modular forms are often polynomial algebras with finitely many generators, this suggests some polynomiality properties of the non-holomorphic free energies [32]. This has led to many recent progress on the computation of Gromov-Witten invariants of quintic Calabi-Yau 3-folds.

Unfortunately the mathematical or geometric definition of the nonholomorphic free energy is lacking in the mathematical literature. According to [3], the nonholomorphic free energy should be closely related to some geometric structures on the relevant moduli spaces. In the genus zero case, it is known that the free energy is related to the special Kähler geometry of the moduli space [4]. In the genus one case, the free energy is related to the $t t^{*}$-geometry of the moduli space $[6,9]$ and the theory of analytic torsions $[2,5]$. In the higher genus case, the nonholomorphic free energy is supposed to be a suitable non-holomorphic section of a holomorphic line bundle on the moduli space [3]. Such an approach depends heavily on the special properties of Calabi-Yau 3-folds.

It is interesting to further clarify the geometric meaning of non-holomorphic free energy, and furthermore, to find analogue of holomorphic anomaly equation for Gromov-Witten theory of general symplectic manifolds or other related theories. The formalism we develop in this paper shed some lights on this problem. In fact, our formalism is inspired by the direct integration approach to holomorphic anomaly equations, and it does not need to start with a Calabi-Yau 3-fold.

Let us briefly recall the direct integration approach. According to [3], the nonholomorphic free energy should count the contributions from degenerate Riemann surfaces, and so it should involve the boundary strata of the Deligne-Mumford moduli spaces of algebraic curves. Witten [30] interpreted the holomorphic anomaly equation from the point of view of geometric quantization of symplectic vector spaces and background independence. Inspired by this work, Aganagic, Bouchard and Klemm [1] obtained via change of 
polarizations the following general form for $\widehat{F}_{g}$ :

$$
\widehat{F}_{g}(t, \bar{t})=F_{g}(t)+\Gamma_{g}\left(\Delta^{I J}, \partial_{I_{1}} \cdots \partial_{I_{n}} F_{r<g}(t)\right),
$$

in some particular cases, $\Delta^{I J}$ can be taken to be $-(\tau-\bar{\tau})^{I J}$, where $\tau=\left(\tau_{i j}\right)=\left(\frac{\partial^{2} F_{0}}{\partial t_{i} \partial t_{j}}\right)$. These particular cases were interpreted for matrix models using Eynard-Orantin topological recursion by Eynard, Mariño and Orantin [17]. Furthermore, they also represented the partition function as a formal Gaussian integral [17, (4.27)] and presented the Feynman graphs and Feynman rules for the terms that contribute to $\Gamma_{g}$. These results have been generalized to other models by Grimm, Klemm, Mariño and Weiss [19]. These authors reformulated the holomorphic anomaly equation as a quadratic recursion relation for the derivative of $\widehat{F}_{g}$ with respective to the propagators $\Delta^{I J}$ (cf. [19, (7.50)]:

$$
\frac{\partial \widehat{F}_{g}}{\partial \Delta^{I J}}=\frac{1}{2} D_{I} \partial_{J} \widehat{F}_{g-1}+\frac{1}{2} \sum_{r=1}^{g-1} \partial_{I} \widehat{F}_{r} \partial_{J} \widehat{F}_{g-r} .
$$

From the consideration of modularity of $\widehat{F}_{g}$, the propagators $\Delta^{I J}$ has the form

$$
\Delta^{I J}=\frac{1}{\sqrt{-1}}\left((\tau-\bar{\tau})^{-1}\right)^{I J}+\mathcal{E}^{I J},
$$

where $\mathcal{E}^{I J}$ is a holomorphic function. They also derived the formal Gaussian integral representation of the partition function and the Feynman expansions for $\widehat{F}_{g}$.

It is clear that our formalism is inspired by $[1,17,19]$. The results in these works when the propagators are

$$
\kappa=-(\tau-\bar{\tau})^{-1}
$$

are special cases of our formalism. Indeed, the condition (4.21) clearly holds for such propagators, and so our formalism can be applied to the free energy $F_{g}$ of Gromov-Witten theory of a compact Calabi-Yau 3-fold to get a sequence $\widehat{F}_{g}$ that satisfies the holomorphic anomaly equations. It is remarkable that the application of our formalism does not need the Calabi-Yau condition, and so it applies to the Gromov-Witten theory of any compact symplectic manifold, any Gromov-Witten type theory as the FJRW theory.

Example 5.1. For FJRW theory of type $A_{1}, F_{0}\left(t_{0}\right)=\frac{t_{0}^{3}}{3 !}$, and so we have

$$
\tau=\frac{\partial^{2} F_{0}}{\partial t_{0}^{2}}=t_{0}
$$

and so

$$
\kappa=\frac{1}{\bar{t}_{0}-t_{0}} .
$$

The free energy functions $F_{g}\left(t_{0}\right)$ are generating functions of intersection numbers on $\overline{\mathcal{M}}_{g, n}$, restricted to the small phase space $t_{i}=0$ for $i>0$. Therefore, for $g \geq 1$,

$$
F_{g}\left(t_{0}\right)=0 \text {. }
$$

Then our construction yields a sequence $\widehat{F}_{g}(t, \bar{t})$ : 
Example 5.2. For the Gromov-Witten theory of $\mathbb{P}^{1}$, the free energy function restricted to the small phase space is given by

$$
\begin{aligned}
& F_{0}\left(t_{0}, t_{1}\right)=\frac{t_{0}^{2} t_{1}}{2}+q e^{t_{1}} \\
& F_{1}\left(t_{0}, t_{1}\right)=-\frac{t_{1}}{24} \\
& F_{g}\left(t_{0}, t_{1}\right)=0, \quad g \geq 2,
\end{aligned}
$$

where $q$ is the degree tracking parameter. The period matrix $\tau$ can be directly computed as follows:

$$
\tau=\left(\begin{array}{cc}
t_{1} & t_{0} \\
t_{0} & q e^{t_{1}}
\end{array}\right)
$$

and so the propagator is

$$
\kappa=-\left(\begin{array}{cc}
t_{1}-\bar{t}_{1} & t_{0}-\bar{t}_{0} \\
t_{0}-\bar{t}_{0} & q e^{t_{1}}-q e^{\bar{t}_{1}}
\end{array}\right)^{-1}
$$

It is an interesting problem to extend our construction to the big phase space that includes all the gravitational descendants of primary observables. It is well-known that Gromov-Witten theory and its various generalizations in the mathematical literature describes what physicists refer to as the topological string theory. The mathematical meaning or definition of the partition function of what physicists refer to as the string theory needs to be clarified at present. For example, for Gromov-Witten theory, the free energy $F_{g}$ depends on infinitely many coupling constants $\left\{t_{i, n}\right\}_{1 \leq i \leq N, n \geq 0}$, we expect the string theory behind it to have free energy $\widehat{F}_{g}$ that also depends on $\left\{\bar{t}_{i, n}\right\}_{1 \leq i \leq N, n \geq 0}$. We hope our construction shed some lights on this problem.

When the propagators take the form

$$
\kappa=-(\tau-\bar{\tau})^{-1}+\mathcal{E}
$$

where $\mathcal{E}$ is a symmetric matrix whose entries are holomorphic functions, the condition (4.21) do not hold in general. This means that we need to modify the diagrammatics we discuss in this paper. We will do this in a subsequent work [29].

\section{Relationship to Eynard-Orantin topological recursion and quantum spectral curves}

In this section we write the wave function of the quantum spectral curve of GukovSułkowski [20] as a summation over stable graphs, and interpret it as a particular realization of the abstract QFT.

\subsection{Graph sum for Eynard-Orantin topological recursion}

The theory of Eynard-Orantin topological recursion was developed by Eynard and Orantin in [16], inspired by the theory of matrix models. The input data of this theory are a 
Riemann surface $\mathcal{C}$ (spectral curve), two holomorphic functions $x$ and $y$ on this curve, and a symmetric bilinear meromorphic 2-form of the 2nd kind $B(p, q)$ (Bergman kernel) on $\mathcal{C} \times \mathcal{C}$ which has a double pole along the diagonal and no other pole. Then a family of meromorphic n-differentials $W_{n}^{(g)}\left(z_{1}, \cdots, z_{n}\right)$ on $\mathcal{C}^{n}$ are defined recursively from these input data. The Eynard-Orantin invariants $W_{n}^{(g)}$ can be represented as a sum over trivalent graphs (with both oriented edges and non-oriented edges) of genus $g$ with $n$ leaves marked by $z_{1}, \cdots, z_{n}$ respectively (see $[16$, section 4.5$]$ ).

In the case of one branch point, Eynard [13] showed that the invariants $W_{n}^{(g)}$ can be expressed in terms of intersection numbers on the moduli space of stable curves $\overline{\mathcal{M}}_{g, n}$. In the case of $N$ branch points, $W_{n}^{(g)}$ can be expressed in terms of intersection numbers on the moduli space of 'colored' stable curves $\overline{\mathcal{M}}_{g, n}^{N}$, see Eynard [14]. The vertices of trivalent graphs in this case are indexed by $\{1, \cdots, N\}$. By cutting these trivalent graphs into clusters of constant indices, summations over these trivalent graphs can be reformulated as summations over labelled stable graphs of type $(g, n)$, see [14, appendix A.3] and Kostov-Orantin [24].

Dunin-Barkowski, Orantin, Shadrin, and Spitz reformulated this diagrammatic representation for the correlation functions $W_{n}^{(g)}$ of the local topological recursion as a summation over decorated stable graphs in [11, section 3]. Now let us recall their results in the rest of this subsection.

For a fixed positive integer $N$, define the analytic functions

$$
x^{i}(z):=z^{2}+a_{i}, \quad y^{i}(z):=\sum_{k=0}^{\infty} h_{k}^{i} z^{k},
$$

and a bi-differential

$$
B^{i j}\left(z, z^{\prime}\right)=\delta_{i j} \cdot \frac{d z \otimes d z^{\prime}}{\left(z-z^{\prime}\right)^{2}}+\sum_{k, l=0}^{\infty} B_{k, l}^{i, j} z^{k} z^{\prime l} d z \otimes d z^{\prime}
$$

in a neighborhood of $0 \in \mathbb{C}$ for $i, j \in\{1,2, \cdots, N\}$, where $\left\{h_{k}^{i}\right\}_{k \in \mathbb{N}}$ are $N$ families of complex numbers called times, and $\left\{B_{k, l}^{i, j}\right\}_{(k, l) \in \mathbb{N}^{2}}$ are $N \times N$ families of complex numbers called jumps. The Eynard-Orantin invariants are defined by

$$
\omega_{0,1}^{i}(z):=0, \quad \omega_{0,2}^{i, j}\left(z, z^{\prime}\right):=B^{i, j}\left(z, z^{\prime}\right),
$$

and the recursion relation

$$
\begin{aligned}
& \omega_{g, n+1}^{i_{0}, i_{1}, \cdots, i_{n}}\left(z_{0}, z_{1}, \cdots, z_{n}\right):=\sum_{j=1}^{N} \operatorname{Res}_{z \rightarrow 0} \frac{\int_{-z}^{z} B^{i_{0}, j}\left(z_{0}, \cdot\right)}{2\left(y^{j}(z)-y^{j}(-z)\right) d x^{j}(z)} . \\
& \left(\omega_{g-1, n+2}^{j, j, i_{1}, \cdots, i_{n}}\left(z,-z, z_{1}, \cdots, z_{n}\right)+\sum_{\substack{0 \leq h \leq g \\
A \sqcup B=\{1, \cdots, n\}}} \omega_{h,|A|+1}^{j, i_{A}}\left(z, z_{A}\right) \omega_{g-h,|B|+1}^{j, i_{B}}\left(-z, z_{B}\right)\right)
\end{aligned}
$$

for every $2 g-2+n>0$, where $i_{A}:=\left\{i_{a_{1}}, \cdots, i_{a_{k}}\right\}$ and $z_{A}:=\left\{z_{a_{1}}, \cdots, z_{a_{k}}\right\}$ for a subset $A=\left\{a_{1}, \cdots, a_{k}\right\}$ of $\{1,2, \cdots, n\}$. 
Following [11], let $\Gamma_{g, n}$ be the set of connected decorated stable graphs of genus $g$, with $n$ ordinary leaves marked by arguments $z_{1}, \cdots, z_{n}$. Let $V(\Gamma), E(\Gamma), H(\Gamma)$ and $L(\Gamma)$ be the set of vertices, edges, half-edges, and leaves of $\Gamma$ respectively. The set of leaves $L(\Gamma)=L^{*}(\Gamma) \sqcup L^{\bullet}(\Gamma)$, where $L^{*}(\Gamma)$ is the set of ordinary leaves, and $L^{\bullet}(\Gamma)$ is the set of dilaton leaves. A half-edge is defined to be either a leaf, or an internal edge together with a choice of one of the two vertices it is attached to. Let $i: V(\Gamma) \rightarrow\{1,2, \cdots, N\}$ be the markings of vertices, and this induces the markings on leaves $i: L(\Gamma) \rightarrow\{1,2, \cdots, N\}$ by assigning $i(l):=i(v)$ if $l$ is a leaf attached to the vertex $v$. The marking on a dilaton leaf is required to be greater than one. For an internal edge $e$, denote by $v_{1}(e), v_{2}(e)$ the two vertices $e$ is attached to, and by $h_{1}(e), h_{2}(e)$ the two corresponding half-edges. For a vertex $v \in V(\Gamma)$, denote by $H(v)$ the set of half-edges incident at $v$. Let $k: H(\Gamma) \rightarrow \mathbb{Z}_{\geq 0}$ be the heights of the half-edges. Define

$$
\omega_{g, n}(\vec{z}):=\sum_{\vec{i}} \omega_{g, n}^{\vec{i}}(\vec{z}) .
$$

Theorem 6.1. ([14], [11, Theorem 3.7]) For $2 g-2+n>0$,

$$
\begin{aligned}
\omega_{g, n}(\vec{z})= & \sum_{\Gamma \in \Gamma_{g, n}} \frac{1}{|\operatorname{Aut}(\Gamma)|} \prod_{v \in V(\Gamma)}\left(-2 h_{1}^{i(v)}\right)^{\chi_{g(v), \operatorname{val}(v)}}\left\langle\prod_{h \in H(v)} \tau_{k(h)}\right\rangle_{g(v), \operatorname{val}(v)} \\
& \cdot \prod_{e \in E(\Gamma)} \check{B}_{k\left(h_{1}(e)\right), k\left(h_{2}(e)\right)}^{i\left(v_{1}(e)\right), i\left(v_{2}(e)\right)} \prod_{l \in L^{*}(\Gamma)} \sum_{j=1}^{N} d \xi_{k(l)}^{i(l)}\left(z_{l}, j\right) \prod_{\lambda \in L^{\bullet}(\Gamma)} \check{h}_{k(\lambda)}^{i(\lambda)},
\end{aligned}
$$

where

$$
\begin{aligned}
\chi_{g(v), \operatorname{val}(v)} & :=2-2 g(v)-\operatorname{val}(v) ; \\
\check{h}_{k}^{i} & :=2(2 k-1) ! ! h_{2 k-1}^{i} ; \\
d \xi_{d}^{i}\left(z_{a}, j\right) & :=\operatorname{Res}_{z \rightarrow 0} \frac{(2 d+1) ! ! d z}{z^{2 d+2}} \int^{z} B^{i, j}\left(z, z_{a}\right) ; \\
\check{B}_{d_{1}, d_{2}}^{i, j} & :=\left(2 d_{1}-1\right) ! !\left(2 d_{2}-1\right) ! ! B_{2 d_{1}, 2 d_{2}}^{i, j} ; \\
\left\langle\prod_{i=1}^{n} \tau_{k_{i}}\right\rangle_{g, n} & :=\int_{\overline{\mathcal{M}}_{g, n}} \psi_{1}^{k_{1}} \psi_{2}^{k_{2}} \cdots \psi_{n}^{k_{n}} .
\end{aligned}
$$

\subsection{Graph sum for quantum spectral curves}

In [20], Gukov and Sułkowski proposed a method to construct the quantum spectral curves using Eynard-Orantin topological recursion. In this subsection we first recall their construction, and then reformulate it as a summation over decorated stable graphs without markings $z_{1}, \cdots, z_{n}$ on ordinary leaves.

Let $\mathbb{C} \times \mathbb{C}$ be the complex plane with coordinates $(u, v)$, equipped with the symplectic form $\omega=\frac{\sqrt{-1}}{\hbar} d u \wedge d v$. Let $A(u, v)$ be a polynomial in $u$ and $v$, then the algebraic curve

$$
\mathcal{C}: \quad A(u, v)=0
$$

is a Lagrangian submanifold in $(\mathbb{C} \times \mathbb{C}, \omega)$. Quantization turns the coordinates $u$ and $v$ into operators $\hat{u}$ and $\hat{v}$ which satisfy the commutation relation $[\hat{v}, \hat{u}]=\hbar$, and algebras 
of functions in $u, v$ into a noncommutative algebra of operators. The quantization of the polynomial $A(u, v)$ is an operator

$$
\widehat{A}=\widehat{A}_{0}+\hbar \widehat{A}_{1}+\hbar^{2} \widehat{A}_{2}+\cdots
$$

where $\widehat{A}_{0}$ is obtained from $A$ by replacing $u, v$ by $\hat{u}, \hat{v}$ respectively.

Inspired by the matrix model origin of the Eynard-Orantin topological recursion, Gukov and Sułkowski defined the following Baker-Akhiezer function (see [20, section 2.1]):

$$
Z(z):=\exp \left(\sum_{n=0}^{\infty} \hbar^{n-1} S_{n}(z)\right),
$$

where

$$
\begin{aligned}
S_{0}(z) & :=\int^{z} v(z) d u(z), \\
S_{1}(z) & :=-\frac{1}{2} \log \frac{d u}{d z} \\
S_{n}(z) & :=\sum_{2 g-1+k=n} \frac{1}{k !} \int^{z} \cdots \int^{z} \omega_{g, k}\left(z_{1}, \cdots, z_{k}\right), \quad n \geq 2,
\end{aligned}
$$

and $\omega_{g, k}\left(z_{1}, \cdots, z_{k}\right)$ are the Eynard-Orantin invariants defined by the spectral curve $u=u(z), v=v(z)$ together with a choice of Bergman kernel $B(p, q)$. Then these authors conjectured that the quantum spectral curve $\widehat{A}$ can be obtained by solving the Schrödinger equation

$$
\widehat{A} Z(z)=0 .
$$

Now let us combine the above construction of $Z(z)$ due to Gukov-Sulkowski and the graph sum (6.1) of Dunin-Barkowski et al. [11] to give a diagrammatic representation for the functions $S_{n}(z)(n \geq 2)$.

It follows from (6.1) that

$$
\begin{aligned}
& \int^{z} \cdots \int^{z} \omega_{g, k}\left(z_{1}, \cdots, z_{k}\right) \\
= & \sum_{\Gamma \in \Gamma_{g, k}} \frac{1}{|\operatorname{Aut}(\Gamma)|} \prod_{v \in V(\Gamma)}\left(-2 h_{1}^{i(v)}\right)^{\chi_{g(v), \operatorname{val}(v)}}\left\langle\prod_{h \in H(v)} \tau_{k(h)}\right\rangle_{g(v), \operatorname{val}(v)} \\
& \cdot \prod_{e \in E(\Gamma)} \check{B}_{k\left(h_{1}(e)\right), k\left(h_{2}(e)\right)}^{i\left(v_{1}(e), i\left(v_{2}(e)\right)\right.} \prod_{l \in L^{*}(\Gamma)} \int^{z}\left(\sum_{j=1}^{N} d \xi_{k(l)}^{i(l)}\left(z_{l}, j\right)\right) \prod_{\lambda \in L^{\bullet}(\Gamma)} \check{h}_{k(\lambda)}^{i(\lambda)} .
\end{aligned}
$$

Therefore $S_{n}$ can be represented as a summation over connected decorated stable graphs of type $(g, k)$ with $2 g-1+k=n$. Notice here the weight of every ordinary leaf $l \in L^{*}(\Gamma)$ is given by

$$
\int^{z}\left(\sum_{j=1}^{N} d \xi_{k(l)}^{i(l)}\left(z_{l}, j\right)\right)
$$


which is independent of $z_{l}$. Thus we now obtain an enumeration problem of decorated stable graphs where we do not mark $z_{1}, \cdots, z_{n}$ on the ordinary leaves, i.e., we do not distinguish these external edges.

Let $\widetilde{\Gamma}_{g, n}$ be the set of decorated stable graphs obtained from decorated stable graphs in $\Gamma_{g, n}$ by forgetting the markings $z_{1}, \cdots, z_{n}$ on ordinary leaves, then we have the following:

Theorem 6.2. For every $2 g-2+k>0$, we have:

$$
\begin{aligned}
& \frac{1}{k !} \int^{z} \cdots \int^{z} \omega_{g, k}\left(z_{1}, \cdots, z_{k}\right) \\
= & \sum_{\widetilde{\Gamma} \in \widetilde{\Gamma}_{g, k}} \frac{1}{|\operatorname{Aut}(\widetilde{\Gamma})|} \prod_{v \in V(\widetilde{\Gamma})}\left(-2 h_{1}^{i(v)}\right)^{\chi_{g(v), \operatorname{val}(v)}}\left\langle\prod_{h \in H(v)} \tau_{k(h)}\right\rangle_{g(v), \operatorname{val}(v)} \\
& \cdot \prod_{e \in E(\widetilde{\Gamma})} \check{B}_{k\left(h_{1}(e)\right), k\left(h_{2}(e)\right)}^{i\left(v_{1}(e), i\left(v_{2}(e)\right)\right.} \prod_{l \in L^{*}(\widetilde{\Gamma})} \int^{z}\left(\sum_{j=1}^{N} d \xi_{k(l)}^{i(l)}(z, j)\right) \prod_{\lambda \in L^{\bullet}(\widetilde{\Gamma})} \check{h}_{k(\lambda)}^{i(\lambda)} .
\end{aligned}
$$

Proof. Let $\widetilde{\Gamma} \in \widetilde{\Gamma}_{g, k}$ be a decorated stable graph of type $(g, k)$, whose ordinary leaves are unmarked. Denote by $\mathcal{S}_{\widetilde{\Gamma}}$ the set of decorated stable graphs $\Gamma \in \Gamma_{g, k}$, such that $\widetilde{\Gamma}$ can be obtained from $\Gamma$ by forgetting the markings $z_{1}, \cdots, z_{k}$ on the ordinary leaves of $\Gamma$.

Since we already have (6.6), it suffices to show that

$$
\frac{1}{k !} \cdot \sum_{\Gamma \in \mathcal{S}_{\widetilde{\Gamma}}} \frac{1}{|\operatorname{Aut}(\Gamma)|}=\frac{1}{|\operatorname{Aut}(\widetilde{\Gamma})|} .
$$

Now let us prove the above equality. First observe that for every $\Gamma, \Gamma^{\prime} \in \mathcal{S}_{\Gamma}$, we have $|\operatorname{Aut}(\Gamma)|=\left|\operatorname{Aut}\left(\Gamma^{\prime}\right)\right|$. Thus we only need to check

$$
\frac{1}{k !} \cdot \frac{\left|\mathcal{S}_{\widetilde{\Gamma}}\right|}{|\operatorname{Aut}(\Gamma)|}=\frac{1}{|\operatorname{Aut}(\widetilde{\Gamma})|}
$$

for an arbitrary $\Gamma \in \mathcal{S}_{\widetilde{\Gamma}}$.

Let the permutation group $\mathcal{S}_{k}$ act on the set $\mathcal{S}_{\widetilde{\Gamma}}$ by permuting the markings $z_{1}, \cdots, z_{n}$ on ordinary leaves. This action is clearly transitive, thus

$$
\left|\mathcal{S}_{\widetilde{\Gamma}}\right|=\frac{\left|\mathcal{S}_{k}\right|}{|H|}=\frac{k !}{|H|},
$$

where $H$ consists of the permutations of the markings that preserves $\Gamma$.

On the other hand, the group $\operatorname{Aut}(\Gamma)$ acts transitively on $H$, and the invariant subgroup is exactly isomorphic to $\operatorname{Aut}(\widetilde{\Gamma})$, thus

$$
\frac{|\operatorname{Aut}(\widetilde{\Gamma})|}{|\operatorname{Aut}(\Gamma)|}=|H|
$$

Then (6.11) and (6.12) give us (6.10).

As a corollary, we obtain the following graph sum for $S_{n}(z)(g \geq 2)$ : 
Corollary 6.1. For every $g \geq 2$, we have

$$
\begin{aligned}
S_{n}(z)= & \sum_{\substack{\widetilde{\Gamma} \in \leq \widetilde{\Gamma}_{g, k} \\
2 g-1+k=n}} \frac{1}{|\operatorname{Aut}(\widetilde{\Gamma})|} \prod_{v \in V(\widetilde{\Gamma})}\left(-2 h_{1}^{i(v)}\right)^{\chi_{g(v), \operatorname{val}(v)}}\left\langle\prod_{h \in H(v)} \tau_{k(h)}\right\rangle_{g(v), \operatorname{val}(v)} \\
& \cdot \prod_{e \in E(\widetilde{\Gamma})} \check{B}_{k\left(h_{1}(e)\right), k\left(h_{2}(e)\right)}^{i\left(v_{1}(e)\right), i\left(v_{2}(e)\right)} \prod_{l \in L^{*}(\widetilde{\Gamma})} \int^{z}\left(\sum_{j=1}^{N} d \xi_{k(l)}^{i(l)}(z, j)\right) \prod_{\lambda \in L^{\bullet}(\widetilde{\Gamma})} \check{h}_{k(\lambda)}^{i(\lambda)} .
\end{aligned}
$$

\subsection{Recursion relations for $S_{n}(z)$}

In last subsection we have represented the function $S_{n}(z)(n \geq 2)$ as a summation over decorated stable graphs (without markings $z_{1}, \cdots, z_{n}$ on ordinary leaves). Therefore it is natural to understand this summation as a particular realization of the abstract quantum field theory.

Here the stable graphs we need are those obtained from the connected stable graphs with labels $\{1,2, \cdots, N\}$ on half-edges (in the sense of section 3 ) by adding some dilaton leaves, and assigning a height $k: H(\widetilde{\Gamma}) \rightarrow \mathbb{Z}_{\geq 0}$. From (6.8) we know that the weight of such a stable graph $\Gamma$ is

$$
\begin{aligned}
\omega_{\widetilde{\Gamma}}:= & \prod_{v \in V(\widetilde{\Gamma})}\left(-2 h_{1}^{i(v)}\right)^{\chi_{g(v), \operatorname{val}(v)}}\left\langle\prod_{h \in H(v)} \tau_{k(h)}\right\rangle_{g(v), \operatorname{val}(v)} \\
& \cdot \prod_{e \in E(\widetilde{\Gamma})} \check{B}_{k\left(h_{1}(e)\right), k\left(h_{2}(e)\right)}^{i\left(v_{1}(e)\right), i\left(v_{2}(e)\right)} \prod_{l \in L^{*}(\widetilde{\Gamma})} \int^{z}\left(\sum_{j=1}^{N} d \xi_{k(l)}^{i(l)}(z, j)\right) \prod_{\lambda \in L^{\bullet}(\widetilde{\Gamma})} \check{h}_{k(\lambda)}^{i(\lambda)},
\end{aligned}
$$

if for every vertex $v \in V(\widetilde{\Gamma})$, the half-edges incident at $v$ have the same index $i(v)$. If not, we assign $\omega_{\widetilde{\Gamma}}:=0$ (by the definition of decorated stable graphs).

Denote by $\widetilde{\mathcal{G}}_{g, n}^{c}(N)$ all such stable graphs of type $(g, n)$, and $\widetilde{\mathcal{G}}_{g ; l_{1}, \cdots, l_{N}}^{c}(N) \subset \widetilde{\mathcal{G}}_{g, n}^{c}(N)$ the subset consisting of all such graphs of genus $g$, with $l_{j}$ external edges labelled by $j$ for every $j \in\{1, \cdots, N\}$ (we have $l_{1}+\cdots+l_{N}=n$ ). The $n$-point function of genus $g$ in this case is

$$
\widetilde{F}_{g ; l_{1}, \cdots, l_{N}}(z):=\frac{1}{n !} \int^{z} \cdots \int^{z} \omega_{g, n}^{\vec{i}}\left(z_{1}, \cdots, z_{n}\right)=\sum_{\widetilde{\Gamma} \in \widetilde{\mathcal{G}}_{g ; l_{1}, \cdots, l_{N}}^{c}(N)} \frac{1}{|\operatorname{Aut}(\widetilde{\Gamma})|} \omega_{\widetilde{\Gamma}},
$$

where $\vec{i}=\left(i_{0}, \cdots, i_{n-1}\right)$ is a sequence such that $j$ appears exactly $l_{j}$ times for every $j \in\{1,2, \cdots, N\}$. We also define

$$
\widetilde{F}_{g, n}(z):=\frac{1}{n !} \int^{z} \cdots \int^{z} \omega_{g, n}\left(z_{1}, \cdots, z_{n}\right)=\sum_{\widetilde{\Gamma}_{\in} \in \widetilde{\mathcal{G}}_{g, n}^{c}} \frac{1}{|\operatorname{Aut}(\widetilde{\Gamma})|} \omega_{\widetilde{\Gamma}}
$$

for every $2 g-2+n$ and $n>0$. Then clearly

$$
\widetilde{F}_{g, n}(z)=\sum_{\left(l_{1}, \cdots, l_{N}\right)} \widetilde{F}_{g ; l_{1}, \cdots, l_{N}}(z),
$$


and the functions $S_{n}(z)$ are given by

$$
S_{n}(z)=\sum_{2 g-1+k=n} \widetilde{F}_{g, k}(z) .
$$

Notice that the recursion relation for abstract $n$-point functions in Theorem 3.2 can be naturally generalized to the case of stable graphs in this case, therefore we obtain the following recursion relation for $\widetilde{F}_{g ; l_{1}, \cdots, l_{N}}(z)$ :

Theorem 6.3. For $2 g-2+\sum l_{j}>0$, we have

$$
\begin{aligned}
& \widetilde{K}_{i j} \widetilde{F}_{g ; l_{1}, \cdots, l_{N}}=\widetilde{D}_{i} \widetilde{D}_{j} \widetilde{F}_{g-1 ; l_{1}, \cdots, l_{N}}+\sum_{\substack{g_{1}+g_{2}=g \\
p_{k}+q_{k}=l_{k}}} \widetilde{D}_{i} \widetilde{F}_{g_{1} ; p_{1}, \cdots, p_{N}} \widetilde{D}_{j} \widetilde{F}_{g_{2} ; q_{1}, \cdots, q_{N}}, \quad i \neq j ; \\
& \widetilde{K}_{i i} \widetilde{F}_{g ; l_{1}, \cdots, l_{N}}=\frac{1}{2}\left(\widetilde{D}_{i} \widetilde{D}_{i} \widetilde{F}_{g-1 ; l_{1}, \cdots, l_{N}}+\sum_{\begin{array}{c}
g_{1}+g_{2}=g \\
p_{k}+q_{k}=l_{k}
\end{array}} \widetilde{D}_{i} \widetilde{F}_{g_{1} ; p_{1}, \cdots, p_{N}} \widetilde{D}_{i} \widetilde{F}_{g_{2} ; q_{1}, \cdots, q_{N}}\right),
\end{aligned}
$$

where $\widetilde{K}_{i j}$ and $\widetilde{D}_{i}$ are realizations of the edge-cutting operators $K_{i j}$ and the edge-adding operators $\mathcal{D}_{i}$ (see section 3.1) respectively.

Now let us take summation over all sequences $\left(l_{1}, \cdots, l_{N}\right)$. We get:

Corollary 6.2. For evry $2 g-2+n>0$, we have

$$
\begin{aligned}
& \widetilde{K}_{i j} \widetilde{F}_{g, n}=\widetilde{D}_{i} \widetilde{D}_{j} \widetilde{F}_{g-1, n}+\sum_{\substack{g_{1}+g_{2}=g \\
n_{1}+n_{2}=n}} \widetilde{D}_{i} \widetilde{F}_{g_{1}, n_{1}} \widetilde{D}_{j} \widetilde{F}_{g_{2}, n_{2}}, \quad i \neq j ; \\
& \widetilde{K}_{i i} \widetilde{F}_{g, n}=\frac{1}{2}\left(\widetilde{D}_{i} \widetilde{D}_{i} \widetilde{F}_{g-1, n}+\sum_{\substack{g_{1}+g_{2}=g \\
n_{1}+n_{2}=n}} \widetilde{D}_{i} \widetilde{F}_{g_{1}, n_{1}} \widetilde{D}_{i} \widetilde{F}_{g_{2}, n_{2}}\right) .
\end{aligned}
$$

The above recursion relations for $\widetilde{F}_{g, n}$ give us a way to compute $S_{n}(z)$ recursively. According to [20], the functions $S_{n}(z)$ are supposed to satisfy some recursion relations, and one may expect that the Schrödinger equation (6.5) for this quantum spectral curve can be obtained from these recursion relations, i.e., the operator $\widehat{A}=\widehat{A}_{0}+\hbar \widehat{A}_{1}+\cdots$ can be solved from these relations.

In the next section, we will present two examples where the quadratic recursion for $S_{n}(z)$ can be written down explicitly (see section 7.3, section 7.4). In these two examples, the partition functions are known to be some one-dimensional formal Gaussian integrals, thus our formalism of the abstract QFT and its realization can be applied directly to this formal Gaussian integral to derive these quadratic recursions. The edge-cutting and edge-adding operators are realized by some differential operators. We will see that these recursions are indeed equivalent to the Schrödinger equations.

However, in general it is not easy to find explicit expressions for these quadratic recursion relations. For a given E-O topological recursion, the realization of the edge-cutting and edge-adding operators can be complicated, and the recursions for $S_{n}(z)$ may be difficult to write down explicitly. We hope to return to this problem in the future. 


\section{Application to topological 1D gravity and quantum spectral curves}

In this section we present another class of applications of our formalism. We will apply it to topological 1D gravity [34]. The propagator in this case is holomorphic. By making particular choices of coupling constants, this gives us examples concerning the quantum spectral curves [20].

\subsection{Some results in topological $1 \mathrm{D}$ gravity}

In [34], the second author defined the partition function of the topological 1D gravity as a formal Gaussian integral:

$$
Z^{1 \mathrm{D}}=\frac{1}{\left(2 \pi \lambda^{2}\right)^{\frac{1}{2}}} \int d x \exp \left[\frac{1}{\lambda^{2}} S(x)\right]
$$

where the action $S(x)$ is

$$
S(x)=-\frac{1}{2} x^{2}+\sum_{n \geq 1} t_{n-1} \frac{x^{n}}{n !} .
$$

The following explicit formula is given in [34, Prop. 4.5]:

$$
Z^{1 \mathrm{D}}=\sum_{n \geq 0} \sum_{\sum_{j=1}^{k} m_{j}=2 n} \frac{(2 n-1) ! !}{\prod_{j=1}^{k}(j !)^{m_{j}} m_{j} !} \lambda^{2 n-2 \sum_{j=1}^{k} m_{j}} \cdot \prod_{j=1}^{k} t_{j-1}^{m_{j}} .
$$

Using Wick's theorem, it is also straightforward to write down the Feynman graphs and the Feynman rules

$$
Z^{1 \mathrm{D}}=\sum_{\Gamma \in \mathcal{G}} \frac{1}{|\operatorname{Aut}(\Gamma)|} \prod_{v \in V(\Gamma)} \lambda^{\operatorname{val}(v)-2} t_{\mathrm{val}(v)-1},
$$

where the sum is taken over the set $\mathcal{G}$ all possible graphs, with the following Feynman rules:

$$
\begin{aligned}
& w(v)=\lambda^{\operatorname{val}(v)-2} t_{\operatorname{val}(v)-1}, \\
& w(e)=1 .
\end{aligned}
$$

One of the results obtained in [34] is that after a natural change of variables, the free energy functions $F_{g}$ have nice simple expressions. This coordinate change can be obtained by the idea of renormalization. Alternatively, one can formally apply the stationary phase method and find the critical point of $S(x)$ and consider the Taylor expansion of $S(x)$ at its critical point. This gives us the following change of coordinates:

$$
\begin{aligned}
I_{0} & =\sum_{k=1}^{\infty} \frac{1}{k} \sum_{p_{1}+\cdots+p_{k}=k-1} \frac{t_{p_{1}}}{p_{1} !} \cdots \frac{t_{p_{k}}}{p_{k} !}, \\
I_{k} & =\sum_{n \geq 0} t_{n+k} \frac{I_{0}^{n}}{n !}, \quad k \geq 1,
\end{aligned}
$$

and

$$
t_{k}=\sum_{n=0}^{\infty} \frac{(-1)^{n} I_{0}^{n}}{n !} I_{n+k}
$$


where $I_{0}$ is the critical point of $S(x)$, and $I_{n}-\delta_{n, 1}(n \geq 1)$ are the Taylor coefficients at $x=I_{0}$. Using the new coordinate $\left\{I_{k}\right\}_{k \geq 0}$, the action (7.2) can be rewritten as

$$
S(x)=\sum_{k=0}^{\infty} \frac{(-1)^{k}}{(k+1) !}\left(I_{k}+\delta_{k, 1}\right) I_{0}^{k+1}+\sum_{n=2}^{\infty} \frac{\left(I_{n-1}-\delta_{n, 2}\right)}{n !}\left(x-I_{0}\right)^{n} .
$$

Then the partition function (7.1) can be rewritten in terms of another formal Gaussian integral:

$$
\begin{aligned}
Z^{1 \mathrm{D}}= & \exp \left[\frac{1}{\lambda^{2}} \sum_{k=0}^{\infty} \frac{(-1)^{k}}{(k+1) !}\left(I_{k}+\delta_{k, 1}\right) I_{0}^{k+1}+\frac{1}{2} \log \frac{1}{1-I_{1}}\right] \\
& \cdot \frac{1}{\left(\pi \lambda^{2}\right)^{\frac{1}{2}}} \int d x \exp \left[\frac{1}{\lambda^{2}}\left(-\frac{1}{2} x^{2}+\sum_{n \geq 3} \frac{I_{n-1}}{\left(1-I_{1}\right)^{\frac{n}{2}}} \cdot \frac{x^{n}}{n !}\right)\right] .
\end{aligned}
$$

This is interpreted in [34] as giving a mean field theory of the topological $1 \mathrm{D}$ gravity. Using this new equivalent theory, the free energy $F^{1 \mathrm{D}}=\sum_{g \geq 0} \lambda^{2 g-2} F_{g}^{1 \mathrm{D}}=\log \left(Z^{1 \mathrm{D}}\right)$ of this theory can be written down as follows:

$$
\begin{aligned}
F_{0}^{1 \mathrm{D}} & =\sum_{k=0}^{\infty} \frac{(-1)^{k}}{(k+1) !}\left(I_{k}+\delta_{k, 1}\right) I_{0}^{k+1}, \\
F_{1}^{1 \mathrm{D}} & =\frac{1}{2} \log \frac{1}{1-I_{1}}, \\
F_{g}^{1 \mathrm{D}} & =\sum_{\sum_{j=2}^{2 g-1}}\left\langle\tau_{2}^{l_{2}} \cdots \tau_{2 g-1}^{l_{2 g-1}}\right\rangle_{g} \prod_{j=2}^{2 g-1} \frac{1}{l_{j} !}\left(\frac{I_{j}}{\left(1-I_{1}\right)^{(j+1) / 2}}\right)^{l_{j}}, g \geq 2 .
\end{aligned}
$$

By rewriting the theory in the new coordinates $\left\{I_{k}\right\}$, the free energy $F^{1 \mathrm{D}}$ has a different Feynman diagram expansion [34, (130)]:

$$
F_{g}^{1 \mathrm{D}}=\sum_{\Gamma \in \mathcal{G}_{g, 0}^{c, 0}} \frac{1}{|\operatorname{Aut}(\Gamma)|} \prod_{v \in V(\Gamma)} I_{\mathrm{val}(v)-1} \cdot \prod_{e \in E(\Gamma)} \frac{1}{1-I_{1}}
$$

for $g \geq 2$, where $\mathcal{G}_{g, 0}^{c, 0}$ is the subset of $\mathcal{G}_{g, 0}^{c}$ consists of stable graphs whose vertices are all of genus 0 .

\subsection{Topological 1D gravity as realization of the diagrammatics of stable graphs}

Now we see that the Feynman rules (7.12) is indeed a special case of the realization of diagrammatics of stable graphs described in section 4 . I.e., $F_{g}^{1 \mathrm{D}}=\widehat{F}_{g}(g \geq 2)$ for suitable $\kappa$ and a suitable sequence $F_{g}(X)$ for $g \geq 0$. By comparing (7.12) with the Feynman rules (4.4), it is clear that for topological $1 \mathrm{D}$ gravity, the propagator is taken to be

$$
\kappa=\frac{1}{1-I_{1}} .
$$


Since in (7.12), the summation is taken over stable graphs with only genus zero vertices, so we have $F_{g}(X)=0$ for $g \geq 1$, and

$$
F_{0}^{(\operatorname{val}(v))}(X)=I_{\operatorname{val}(v)-1},
$$

where $\operatorname{val}(v) \geq 3$. In other words, we need a function $F_{0}(X)$ such that

$$
\frac{d^{n}}{d X^{n}} F_{0}(X)=I_{n-1}
$$

for $n \geq 3$. Since $I_{n-1}$ involves an infinite set of indeterminates $t_{0}, t_{1}, \ldots$, it is not clear how to choose one of them or a combination of them to be $X$. We will take a different approach as follows. Regard $\left\{I_{k}\right\}_{k \geq 0}$ as some parameters, and $X$ as a new independent variable. Now we define a function $F_{0}(X)$ by

$$
F_{0}(X)=\sum_{k=0}^{\infty} \frac{(-1)^{k}}{(k+1) !}\left(I_{k}+\delta_{k, 1}\right) I_{0}^{k+1}+\sum_{n=2}^{\infty} \frac{\left(I_{n-1}-\delta_{n, 2}\right)}{n !}\left(X-I_{0}\right)^{n} .
$$

Write $\tilde{X}=X-I_{0}$, then

$$
F_{0}\left(I_{0}+\tilde{X}\right)=\sum_{k=0}^{\infty} \frac{(-1)^{k}}{(k+1) !}\left(I_{k}+\delta_{k, 1}\right) I_{0}^{k+1}+\sum_{n=2}^{\infty} \frac{\left(I_{n-1}-\delta_{n, 2}\right)}{n !} \tilde{X}^{n} .
$$

This is equivalent to say

$$
\begin{aligned}
F_{0}\left(I_{0}\right) & =\sum_{k=0}^{\infty} \frac{(-1)^{k}}{(k+1) !}\left(I_{k}+\delta_{k, 1}\right) I_{0}^{k+1}, \\
\left.\frac{d F_{0}(X)}{d X}\right|_{X=I_{0}} & =0 \\
\left.\frac{d^{n} F_{0}(X)}{d X^{n}}\right|_{X=I_{0}} & =I_{n-1}-\delta_{n, 2}, \quad n \geq 2 .
\end{aligned}
$$

Now using the formalism in section 4 , we can construct a sequence $\widehat{F}_{g}(X)$ from $F(X)=$ $\lambda^{-2} \cdot F_{0}(X)$, and the propagator

$$
\kappa=-\frac{1}{F_{0}^{\prime \prime}(X)}
$$

which clearly satisfies the condition (4.18). Hence (7.13), (7.14)and (7.15) hold when $X=I_{0}$. For $g \geq 2$ we have

$$
\widehat{F}_{g}(X)=\sum_{\Gamma \in \mathcal{G}_{g, 0}^{c, 0}} \frac{1}{|\operatorname{Aut}(\Gamma)|}\left(\prod_{v \in V(\Gamma)} F_{0}^{(\operatorname{val}(v))}(X)\right) \cdot \kappa^{|E(\Gamma)|} .
$$

Then evaluate this expression at $X=I_{0}$ using (7.18) and (7.19), we get

$$
\begin{aligned}
\widehat{F}_{g}\left(I_{0}\right) & =\sum_{\Gamma \in \mathcal{G}_{g, 0}^{c, 0}} \frac{1}{|\operatorname{Aut}(\Gamma)|}\left(\left.\prod_{v \in V(\Gamma)} F_{0}^{(\operatorname{val}(v))}(X)\right|_{X=I_{0}}\right) \cdot\left(-\frac{1}{F_{0}^{\prime \prime}(X) \mid X=I_{0}}\right)^{|E(\Gamma)|} \\
& =\sum_{\Gamma \in \mathcal{G}_{g, 0}^{c, 0}} \frac{1}{|\operatorname{Aut}(\Gamma)|}\left(\prod_{v \in V(\Gamma)} I_{\operatorname{val}(v)-1}\right) \cdot\left(\frac{1}{1-I_{1}}\right)^{|E(\Gamma)|},
\end{aligned}
$$


this is exactly the Feynman rule (7.12), i.e., we have proved the following

Theorem 7.1. The free energy of the topological $1 D$ gravity can be realized by the abstract free energy:

$$
F_{g}^{1 \mathrm{D}}\left(I_{0}, I_{1}, I_{2}, \cdots\right)=\left.\widehat{F}_{g}(X)\right|_{X=I_{0}}
$$

for $g \geq 2$. Here $F=\lambda^{-2} F_{0}$ and $\kappa$ are given by (7.16) and (7.19) respectively.

Example 7.1. $F_{2}^{1 \mathrm{D}}$ and $F_{3}^{1 \mathrm{D}}$ are explicitly given by:

$$
\begin{aligned}
& F_{2}^{1 \mathrm{D}}=\frac{1}{12} \bigodot+\frac{1}{8} \bigcirc \infty+\frac{1}{8} \bigcirc \infty \\
& F_{3}^{1 \mathrm{D}}=\frac{1}{48} \curvearrowleft+\frac{1}{48} \Longleftrightarrow+\frac{1}{16} \bigcirc_{0} \infty+\frac{1}{16} \gamma_{0} \infty \\
& +\frac{1}{12} \bigcirc_{0}+\frac{1}{8} \bigcirc_{0} \rightleftharpoons+\frac{1}{16} \bigcirc_{0} \wp_{\infty} \infty \\
& \left.+\frac{1}{12} \bigcirc_{0} \propto+\frac{1}{8} \bigcap_{0}+\frac{1}{8} \bigotimes_{0}+\frac{1}{48} \bigcirc_{0}\right\}_{\infty} \\
& +\frac{1}{24} \bigcap_{0}+\frac{1}{16} \rightleftarrows+\frac{1}{16} \bigcirc_{0} \cdots \infty+\frac{1}{8} \bigcirc_{0}
\end{aligned}
$$

or,

$$
\begin{aligned}
& F_{2}^{1 \mathrm{D}}=\frac{5}{24} I_{2}^{2} \kappa^{3}+\frac{1}{8} I_{3} \kappa^{2}, \\
& F_{3}^{1 \mathrm{D}}=\frac{1}{48} I_{5} \kappa^{3}+\left(\frac{1}{12} I_{3}^{2}+\frac{7}{48} I_{2} I_{4}\right) \kappa^{4}+\frac{25}{48} I_{2}^{2} I_{3} \kappa^{5}+\frac{5}{16} I_{2}^{4} \kappa^{6},
\end{aligned}
$$

where $\kappa=\frac{1}{1-I_{1}}$.

As a corollary to Theorem 4.2 and Theorem 7.1, we get a quadratic recursion relation for $F^{1 \mathrm{D}}$ as follows. First we have

$$
\partial_{\kappa} \widehat{F}_{g}=\frac{1}{2}\left(\left(\partial_{X}+\kappa F_{0}^{\prime \prime \prime}(X)\right) \partial_{X} \widehat{F}_{g-1}+\sum_{r=1}^{g-1} \partial_{X} \widehat{F}_{r} \partial_{X} \widehat{F}_{g-r}\right),
$$

where $\widehat{F}_{1}=F_{1}^{1 \mathrm{D}}=\frac{1}{2} \log \kappa$, next we restrict to $X=I_{0}$ to get:

Theorem 7.2. For every $g \geq 2$,

$$
\partial_{\kappa} F_{g}^{1 \mathrm{D}}=\frac{1}{2}\left(\left(d_{X}+\kappa I_{2}\right) d_{X} F_{g-1}^{1 \mathrm{D}}+\sum_{r=1}^{g-1} d_{X} F_{r}^{1 \mathrm{D}} \cdot d_{X} F_{g-r}^{1 \mathrm{D}}\right),
$$

where $d_{X}$ is a differential operator such that

$$
d_{X} I_{k}=I_{k+1}, \quad k \geq 1, \quad d_{X} \kappa=\kappa^{2} \cdot I_{2},
$$

i.e., $d_{X}:=\sum_{k \geq 1} I_{k+1} \frac{\partial}{\partial I_{k}}$. 
This theorem provides a way to compute $F_{g}^{1 \mathrm{D}}$ recursively without listing all possible Feynman graphs.

Example 7.2. For $g=4$, we have

$$
\begin{aligned}
2 \frac{\partial F_{4}^{1 \mathrm{D}}}{\partial \kappa}= & \left(d_{X}+\kappa I_{2}\right) \partial_{I_{0}} F_{3}^{1 \mathrm{D}}+2 d_{X} F_{1}^{1 \mathrm{D}} \cdot d_{X} F_{3}^{1 \mathrm{D}}+\left(d_{X} F_{2}^{1 \mathrm{D}}\right)^{2} \\
= & \frac{1}{48} I_{7} \kappa^{3}+\left(\frac{5}{16} I_{2} I_{6}+\frac{25}{48} I_{3} I_{5}+\frac{21}{64} I_{4}^{2}\right) \kappa^{4}+\left(\frac{113}{48} I_{2}^{2} I_{5}+\frac{33}{24} I_{3}^{3}+7 I_{2} I_{3} I_{4}\right) \kappa^{5} \\
& +\left(\frac{3115}{144} I_{2}^{2} I_{3}^{2}+\frac{1127}{96} I_{2}^{3} I_{4}\right) \kappa^{6}+\frac{985}{24} I_{2}^{4} I_{3} \kappa^{7}+\frac{1105}{64} I_{2}^{6} \kappa^{8} .
\end{aligned}
$$

Then $F_{4}^{1 \mathrm{D}}$ can be obtained by integrate with respext with $\kappa$ :

$$
\begin{aligned}
F_{4}^{1 \mathrm{D}}= & \frac{1}{384} I_{7} \kappa^{4}+\left(\frac{1}{32} I_{2} I_{6}+\frac{5}{96} I_{3} I_{5}+\frac{21}{640} I_{4}^{2}\right) \kappa^{5} \\
& +\left(\frac{113}{576} I_{2}^{2} I_{5}+\frac{11}{96} I_{3}^{3}+\frac{7}{12} I_{2} I_{3} I_{4}\right) \kappa^{6}+\left(\frac{445}{288} I_{2}^{2} I_{3}^{2}+\frac{161}{192} I_{2}^{3} I_{4}\right) \kappa^{7} \\
& +\frac{985}{384} I_{2}^{4} I_{3} \kappa^{8}+\frac{1105}{1152} I_{2}^{6} \kappa^{9} .
\end{aligned}
$$

\subsection{Example: the Airy function and quantum Airy curve}

Our first example is the Airy function

$$
Z^{A}(t)=C \int d \eta \exp \left[-\lambda^{-2}\left(-\eta t+\frac{\eta^{3}}{3}\right)\right]
$$

where $C$ is a suitable normalization constant. Let $f(\eta)=-\eta t+\frac{\eta^{3}}{3}$, then the critical points of $f(\eta)$ is $\eta= \pm t^{\frac{1}{2}}$. Denote by $c$ one of the critical points and $\tilde{\eta}=\eta-c$, then

$$
f(\eta)=-(\tilde{\eta}+c) c^{2}+\frac{1}{3}(\tilde{\eta}+c)^{3}=-\frac{2}{3} c^{3}+c \tilde{\eta}^{2}+\frac{1}{3} \tilde{\eta}^{3} .
$$

Therefore in this case

$$
\begin{aligned}
& I_{0}=c, \quad I_{1}=1-2 c, \quad I_{2}=-2 \\
& I_{k}=0, \quad k \geq 3 .
\end{aligned}
$$

And the propagator is

$$
\kappa=\frac{1}{1-I_{1}}=\frac{1}{2 c} .
$$

Using the coordinate change (7.8), this particular point (7.30) in the space of coupling constants is

$$
\begin{aligned}
t_{0} & =c^{2}, \quad t_{1}=1, \quad t_{2}=-2, \\
t_{k} & =0, \quad k \geq 3 .
\end{aligned}
$$

In what follows we will use $\frac{\partial}{\partial c}$ to replace the operator $d_{T}$ since we have $\frac{\partial I_{k}}{\partial c}=I_{k+1}$ for $k \geq 1$ and $\frac{\partial \kappa}{\partial c}=\kappa^{2} I_{2}$. Now using our formalism we can compute the free energies $F_{g}^{A}$ associated with $Z^{A}$. The Feynman graphs of this integral only involves cubic graphs without external edges, whose vertices are all of genus zero. 
Example 7.3. The free energies $F_{0}^{A}$ and $F_{1}^{A}$ are given by

$$
F_{0}^{A}=-\frac{2}{3} c^{3}, \quad F_{1}^{A}=\frac{1}{2} \log \frac{1}{2 c} .
$$

And $F_{2}^{A}$ and $F_{3}^{A}$ are given by

$$
\begin{aligned}
& F_{2}^{A}=\frac{1}{12} \bigcirc+\frac{1}{8} \bigcirc_{0} \infty \text {, } \\
& F_{3}^{A}=\frac{1}{8} \bigcirc_{0} \propto+\frac{1}{16} \bigcirc_{0} \curvearrowleft \infty+\frac{1}{16} \rightleftarrows \\
& +\frac{1}{24} \rightleftharpoons 0+\frac{1}{48} \quad 0
\end{aligned}
$$

or,

$$
F_{2}^{A}=\frac{5}{48 c^{3}}, \quad F_{3}^{A}=\frac{5}{64 c^{6}} .
$$

Using (7.31), for $g \geq 1$ we have

$$
\partial_{\kappa} F_{g}^{A}=\left(-2 c^{2}\right) \cdot \frac{\partial F_{g}^{A}}{\partial c}
$$

thus Theorem 4.2 give us the following recursion relation.

Theorem 7.3. For $g \geq 2$ we have

$$
\frac{\partial F_{g}^{A}}{\partial c}=-\frac{1}{4 c^{2}}\left[\left(\frac{\partial}{\partial c}-\frac{1}{c}\right) \frac{\partial F_{g-1}^{A}}{\partial c}+\sum_{r=1}^{g-1} \frac{\partial F_{r}^{A}}{\partial c} \frac{\partial F_{g-r}^{A}}{\partial c}\right] .
$$

Note that in this case $F_{g}^{A}$ must be a monomial in $\kappa=\frac{1}{2 c}$ of degree $3 g-3$, thus $F_{g}^{A}$ can be solved recursively using this relation. For example,

$$
F_{4}^{A}=\frac{1105}{9216 c^{9}}, \quad F_{5}^{A}=\frac{565}{2048 c^{12}}, \quad \cdots
$$

The recursion relation (7.35) is equivalent to the equation

$$
\left(\left(\hbar \frac{\partial}{\partial t}\right)^{2}-t\right) Z^{A}(t)=0
$$

if we set $\hbar=\lambda^{2}$. This is the Schrödinger equation of the quantum Airy curve.

Now let us recall some results about the quantum Airy curve [20,33] and compare with the above construction. First let $u(z)=\frac{1}{2} z^{2}, v(z)=z$ be a parametrization of the Airy curve

$$
A(u, v)=\frac{1}{2} v^{2}-u=0
$$


then using the Eynard-Orantin topological recursion [16] one may get a family of differentials

$$
W_{g, n}\left(p_{1}, \cdots, p_{n}\right)=\mathcal{W}_{g, n}\left(z_{1}, \cdots, z_{n}\right) d z_{1} \cdots d z_{n} .
$$

These invariants associated to the Airy curve encode the information of the WittenKontsevich tau-function [23, 31, 35]. Following [20], define

$$
Z_{\text {Airy }}=\exp \left(\sum_{n=0}^{\infty} \hbar^{n-1} S_{n}\right)
$$

where $S_{n}$ are defined in (6.4). Then $S_{k}$ can be used to construct the quantization of the Airy curve. The following result was proposed by Gukov-Sułkowski in [20], and proved in [33].

Theorem 7.4. ([33]) The function $Z_{\text {Airy }}$ satisfies

$$
\hat{A} Z_{\text {Airy }}=0
$$

where

$$
\hat{A}=\frac{1}{2} \hat{v}^{2}-\hat{u}=\frac{1}{2}\left(\hbar \partial_{u}\right)^{2}-u
$$

is the quantization of the Airy curve.

One computes

$$
\begin{aligned}
S_{0} & =\frac{1}{3} z^{3}, & S_{1} & =-\frac{1}{2} \log (z), \\
S_{2} & =\frac{5}{24 z^{3}}, & S_{3} & =\frac{5}{16 z^{6}},
\end{aligned}
$$

We make a comparison between this and (7.34), (7.36), then we might simply expect $F_{g}^{A}=S_{g}$ for $g \geq 2$ once we set $z^{3}=2 c^{3}$. In fact, this can be proved by the following recursion relation.

Lemma 7.1. ([33]) For $n>2$, we have

$$
\frac{1}{2} \partial_{u}^{2} S_{n-1}+\partial_{u} S_{0} \cdot \partial_{u} S_{n}+\partial_{u} S_{1} \cdot \partial_{u} S_{n-1}+\frac{1}{2} \sum_{\substack{i+j=n \\ i, j \geq 2}} \partial_{u} S_{i} \cdot \partial_{u} S_{j}=0
$$

This lemma can be obtained from Theorem 7.4 by expanding (7.39) directly as a series in $\hbar$ and comparing the coefficients. Using $z^{3}=2 c^{3}$ and $u=\frac{1}{2} z^{2}$, this recursion is equivalent to (7.35).

In conclusion, for the particular choices (7.30) of coupling constants, the procedure to produce $F_{g}^{A}$ is equivalent to the solution of quantization of Airy curve obtained by the Eynard-Orantin topological recursion. 


\subsection{Example: the Kontsevich-Penner matrix model for $N=1$}

Our next example is the Kontsevich-Penner matrix model for $N=1[23,27]$, which is related to the quantization of the Catalan curve

$$
x=z+\frac{1}{z},
$$

see $[20,25]$. The Schrödinger equation in this case is

$$
\left[\hbar^{2} \frac{\partial^{2}}{\partial t^{2}}+\hbar t \frac{\partial}{\partial t}+(1-\hbar)\right] Z^{C}(t)=0
$$

where the partition function is the formal integral

$$
Z^{C}(t)=\int d \eta \exp \left[-\lambda^{-2}\left(\frac{1}{2} \eta^{2}+\eta t+\log \eta\right)\right]
$$

up to a normalization constant, and we set $\hbar=\lambda^{2}$.

Now let us understand this integral using the model of topological 1D gravity. Write $f(\eta)=\frac{1}{2} \eta^{2}+\eta t+\log (\eta)$, we expand $f(\eta)$ at its critical points $c=\frac{-t \pm \sqrt{t^{2}-4}}{2}$. Let $\tilde{\eta}=$ $\eta-c$, then

$$
\begin{aligned}
f(\eta) & =f(\tilde{\eta}+c) \\
& =\left(-\frac{c^{2}}{2}-1+\log (c)\right)+\frac{1}{2}\left(1-c^{-2}\right) \tilde{\eta}^{2}+\frac{\tilde{\eta}^{3}}{3 c^{3}}-\frac{\tilde{\eta}^{4}}{4 c^{4}}+\frac{\tilde{\eta}^{5}}{5 c^{5}}-\cdots
\end{aligned}
$$

By taking

$$
\kappa=\frac{1}{1-c^{-2}}
$$

and

$$
I_{k}=\frac{(-1)^{k+1} \cdot k !}{c^{k+1}}, \quad k \geq 1,
$$

we see $I_{k}^{\prime}=I_{k+1}$ and $\kappa^{\prime}=\kappa^{2} \cdot I_{2}$ where the prime means taking derivative with respect to $c$. Thus we get a special case of the theory in section 7.2, thus we obtain a sequence of free energies $F_{g}^{C}$. Using the formula for $F_{g}^{C}$ for $g=2,3,4$ given by (7.23) and (7.27), we get:

$$
\begin{aligned}
F_{0}^{C}= & -\frac{c^{2}}{2}-1+\log (c), \quad F_{1}^{C}=\frac{1}{2} \log \frac{1}{1-c^{-2}}, \\
F_{2}^{C}= & \frac{3}{4\left(c^{2}-1\right)^{2}}+\frac{5}{6\left(c^{2}-1\right)^{3}}, \\
F_{3}^{C}= & \frac{5}{2\left(c^{2}-1\right)^{3}}+\frac{10}{\left(c^{2}-1\right)^{4}}+\frac{25}{2\left(c^{2}-1\right)^{5}}+\frac{5}{\left(c^{2}-1\right)^{6}}, \\
F_{4}^{C}= & \frac{105}{8\left(c^{2}-1\right)^{4}}+\frac{507}{5\left(c^{2}-1\right)^{5}}+\frac{6391}{24\left(c^{2}-1\right)^{6}}+\frac{767}{2\left(c^{2}-1\right)^{7}} \\
& +\frac{985}{4\left(c^{2}-1\right)^{8}}+\frac{1105}{18\left(c^{2}-1\right)^{9}} .
\end{aligned}
$$


The quadratic recursion relation in Theorem 4.2 reads

$$
\partial_{\kappa} F_{g}^{C}=\frac{1}{2}\left[\left(\frac{\partial}{\partial c}+\kappa \cdot I_{2}\right) \frac{\partial}{\partial c} F_{g-1}^{C}+\sum_{r=1}^{g-1} \frac{\partial}{\partial c} F_{r}^{C} \cdot \frac{\partial}{\partial c} F_{g-r}^{C}\right], \quad g \geq 2 .
$$

To write this relation completely in terms of $\frac{\partial}{\partial c}$, we need the following lemma.

Lemma 7.2. For $g \geq 2$, we have

$$
\frac{\partial}{\partial c} F_{g}^{C}=\left(\kappa^{2} \cdot I_{2}-\frac{2 \kappa}{c}\right) \partial_{\kappa} F_{g}^{C}
$$

Proof. Using the homogeneity we could see that every monomial in $F_{g}^{1 \mathrm{D}}$ is of the form $a_{g, i_{1} \cdots i_{k}} I_{i_{1}} \cdots I_{i_{k}} \cdot \kappa^{l}$ with $i_{1}+\cdots i_{k}+k=2 l$. Thus

$$
\begin{aligned}
\frac{\frac{\partial}{\partial c}\left(I_{i_{1}} \cdots I_{i_{k}} \kappa^{l}\right)}{\partial_{\kappa}\left(I_{i_{1}} \cdots I_{i_{k}} \kappa^{l}\right)} & =\frac{\sum_{j=1}^{k} I_{i_{1}} \cdots I_{i_{j}+1} \cdots I_{i_{k}} \kappa^{l}+I_{i_{1}} \cdots I_{i_{k}} l \kappa^{l-1} \cdot \kappa^{2} I_{2}}{I_{i_{1}} \cdots I_{i_{k}} l \kappa^{l-1}} \\
& =\frac{1}{l} \sum_{j=1}^{k} \frac{I_{i_{j}+1}}{I_{j}} \cdot \kappa+\kappa^{2} \cdot I_{2} \\
& =\frac{1}{l} \sum_{j=1}^{k} \frac{(-1)\left(i_{j}+1\right)}{c} \cdot \kappa+\kappa^{2} \cdot I_{2} \\
& =-\frac{2}{c} \cdot \kappa+\kappa^{2} \cdot I_{2},
\end{aligned}
$$

which proves the lemma.

Now using this lemma and

$$
\kappa^{2} \cdot I_{2}-\frac{2 \kappa}{c}=-\frac{2 c^{3}}{\left(c^{2}-1\right)^{2}}
$$

we rewrite the recursion relation as follows.

Theorem 7.5. For the Kontsevich-Penner model, for $g \geq 2$, we have

$$
\frac{\partial}{\partial c} F_{g}^{C}=-\frac{c^{3}}{\left(c^{2}-1\right)^{2}}\left[\left(\frac{\partial}{\partial c}-\frac{2}{c\left(c^{2}-1\right)}\right) \frac{\partial}{\partial c} F_{g-1}^{C}+\sum_{r=1}^{g-1} \frac{\partial}{\partial c} F_{r}^{C} \cdot \frac{\partial}{\partial c} F_{g-r}^{C}\right] .
$$

Or in terms of $t=-\left(c+\frac{1}{c}\right)$,

$$
\left(c-\frac{1}{c}\right) \cdot \frac{\partial}{\partial t} F_{g}^{C}=\frac{\partial^{2}}{\partial t^{2}} F_{g-1}^{C}+\sum_{r=1}^{g-1} \frac{\partial}{\partial t} F_{r}^{C} \cdot \frac{\partial}{\partial t} F_{g-r}^{C} .
$$

The second equation in the above theorem is exactly the same as the recursive formula for the quantization of the Catalan curve [25, (4.13)]. Similar to the case of quantum Airy curve, the free energies $F_{g}^{C}$ here can also be constructed from the Catalan curve using the Eynard-Orantin topological recursion. 


\subsection{Example: enumeration of stable graphs with genus zero vertices}

In this subsection we apply topological 1D gravity and our formalism to study the problem of enumeration of stable graphs with only genus zero vertices. In this model, all vertices of the Feynman graphs have valence $\geq 3$. The partition function is given by

$$
Z^{s t}(T, \kappa)=\frac{\kappa^{1 / 2}}{\left(2 \pi \lambda^{2}\right)^{1 / 2}} \int d x \exp \left[\lambda^{-2}\left(T \sum_{n \geq 3} \frac{x^{n}}{n !}-\frac{x^{2}}{2 \kappa}\right)\right] .
$$

Write the free energy $F^{s t}=\log Z^{s t}$ in the following expansion:

$$
F^{s t}(T, \kappa)=\sum_{g \geq 2} \sum_{d=1}^{2 g-2} a_{d}^{g} T^{d} \kappa^{g-1+d}
$$

By $(7.3)$

$$
F^{s t}(T, \kappa)=\log \left(\sum_{k \geq 0} \sum_{l_{i} \geq 3} \sum_{l} \frac{\lambda^{2 l-2 k}}{k ! \cdot l_{1} ! \cdots l_{k} !} \cdot(2 l-1) ! ! \cdot T^{k} \cdot \kappa^{l} \cdot \delta_{l_{1}+\cdots+l_{k}, 2 l}\right) .
$$

The coefficients $a_{d}^{g}$ counts the number of connected stable graphs of genus $g$ with $d$ vertices of marked genus 0 , weighted by the inverse of the orders of their automorphism groups. Such graphs has $g-1+d$ edges. Using this interpretation one gets the following explicit formulas for $a_{d}^{g}, d=1,2$ :

$$
\begin{aligned}
& a_{1}^{g}=\frac{1}{(2 g) ! !}=\frac{1}{2^{g} g !}, \\
& a_{2}^{g}=\frac{1}{2} \sum_{k=1}^{g+1} \sum_{\substack{k+2 l \geq 3 \\
2 g+2-k-2 l \geq 3}} \frac{1}{k ! \cdot(2 l) ! ! \cdot(2 g+2-2 k-2 l) ! !} .
\end{aligned}
$$

By an elementary calculation, one can simplify the second equality to get

$$
a_{2}^{g}=\frac{2^{2 g}-2^{g-1}-(g+1)^{2}}{2^{g} \cdot(g+1) !} .
$$

Since $a_{2 g-2}^{g}$ counts trivalent graphs of genus $g$, it equals to $b_{g-1}$ in $[10,(65),(66)]$, and so

$$
\sum_{g \geq 2} a_{2 g-2}^{g} x^{g-1}=\log \left(\sum_{m=0}^{\infty} \frac{(6 m) !}{(3 m) !(2 m) !}\left(\frac{x}{288}\right)^{m}\right) .
$$

It is interesting to compute $a_{d}^{g}$ for $3 \leq d<2 g-2$. Formula (7.54) and the direct counting of graphs are not very effective for this purpose. We will apply the quadratic recursion relations developed in section 7.2 to derive some recursion relations for $a_{d}^{g}$.

In (7.25) and (7.26), we take $I_{k}=T$ for all $k \geq 1$, and then $d_{X}$ changes to an operator $D$ such that

$$
D T=T, \quad D \kappa=\kappa^{2} T
$$


then we get from (7.25) the following recursion relation:

$$
\partial_{\kappa} F_{g}^{s t}=\frac{1}{2}\left((D+\kappa T) D F_{g-1}^{s t}+\sum_{r=1}^{g-1} D F_{r}^{s t} \cdot D F_{g-r}^{s t}\right),
$$

where we have to add the following convention:

$$
F_{1}^{s t}=\frac{1}{2} \log \kappa .
$$

From this we recursively:

$$
\begin{aligned}
& F_{2}^{s t}=\frac{T \kappa^{2}}{8}+\frac{5 T^{2} \kappa^{3}}{24}, \\
& F_{3}^{s t}=\frac{T \kappa^{3}}{48}+\frac{11 T^{2} \kappa^{4}}{48}+\frac{25 T^{3} \kappa^{5}}{48}+\frac{5 T^{4} \kappa^{6}}{16}, \\
& F_{4}^{s t}=\frac{T \kappa^{4}}{384}+\frac{223 T^{2} \kappa^{5}}{1920}+\frac{515 T^{3} \kappa^{6}}{576}+\frac{1373 T^{4} \kappa^{7}}{576}+\frac{985 T^{5} \kappa^{8}}{384}+\frac{1105 T^{6} \kappa^{9}}{1152} .
\end{aligned}
$$

We have

$$
\begin{aligned}
D F_{g}^{s t}= & \sum_{d} a_{d}^{g} d T^{d} \kappa^{g-1+d}+\sum_{d} a_{d}^{g}(g-1+d) T^{d+1} \kappa^{g+d}, \\
D^{2} F_{g}^{s t}= & \sum_{d} a_{d}^{g} d^{2} T^{d} \kappa^{g-1+d}+\sum_{d} a_{d}^{g}(g-1+d) d T^{d+1} \kappa^{g+d} \\
& +\sum_{d} a_{d}^{g}(g-1+d)(d+1) T^{d+1} \kappa^{g+d}+\sum_{d} a_{d}^{g}(g-1+d)(g+d) T^{d+2} \kappa^{g+d+1} .
\end{aligned}
$$

Then (7.58) gives us

$$
\begin{aligned}
& 2 \sum_{d} a_{d}^{g}(g-1+d) T^{d} \kappa^{g-2+d} \\
= & (D+\kappa T) D F_{g-1}^{s t}+\sum_{r=1}^{g-1} D F_{r}^{s t} D F_{g-r}^{s t} \\
= & \sum_{d} a_{d}^{g-1} d^{2} T^{d} \kappa^{g-2+d}+\sum_{d} a_{d}^{g-1}(g-2+d) d T^{d+1} \kappa^{g-1+d} \\
& +\sum_{d} a_{d}^{g-1}(g-2+d)(d+1) T^{d+1} \kappa^{g-1+d} \\
& +\sum_{d} a_{d}^{g-1}(g-2+d)(g-1+d) T^{d+2} \kappa^{g+d} \\
& +2\left[\sum_{d} a_{d}^{g-1} d T^{d+1} \kappa^{g-1+d}+\sum_{d} a_{d}^{g-1}(g-2+d) T^{d+2} \kappa^{g+d}\right] \\
& +\sum_{r=2}^{g-2}\left(\sum_{d} a_{d}^{r} d T^{d} \kappa^{r-1+d}+\sum_{d} a_{d}^{r}(r-1+d) T^{d+1} \kappa^{r+d}\right) \\
& \cdot\left(\sum_{d} a_{d}^{g-r} d T^{d} \kappa^{g-r-1+d}+\sum_{d} a_{d}^{g-r}(g-r-1+d) T^{d+1} \kappa^{g-r+d}\right)
\end{aligned}
$$


for $g \geq 3$. Therefore the following quadratic recursion relations hold for $a_{d}^{g}$ :

$$
\begin{aligned}
a_{d}^{g}=\frac{1}{2(g-1+d)}\{ & \sum_{d} a_{d}^{g-1} d^{2}+\sum_{d} a_{d-2}^{g-1}(g-4+d)(g-1+d) \\
& +\sum_{d} a_{d-1}^{g-1}[(g-3+d)(2 d-1)+2(d-1)] \\
& +\sum_{r=2}^{g-2}\left(\sum_{d_{1}+d_{2}=d} a_{d_{1}}^{r} a_{d_{2}}^{g-r} d_{1} d_{2}+\sum_{d_{1}+d_{2}=d-1} a_{d_{1}}^{r} a_{d_{2}}^{g-r}\left(r-1+d_{1}\right) d_{2}\right. \\
& +\sum_{d_{1}+d_{2}=d-1} a_{d_{1}}^{r} a_{d_{2}}^{g-r} d_{1}\left(g-r-1+d_{2}\right) \\
& \left.\left.+\sum_{d_{1}+d_{2}=d-2} a_{d_{1}}^{r} a_{d_{2}}^{g-r}\left(r-1+d_{1}\right)\left(g-r-1+d_{2}\right)\right)\right\} .
\end{aligned}
$$

\subsection{Example: enumeration of graphs}

In this subsection we study another example: the model describing enumeration of graphs, not necessarily stable, introduced and studied in [10]. The partition function for this case is

$$
Z^{\mathrm{gr}}(t)=\frac{1}{\sqrt{2 \pi \lambda^{2}}} \int d \eta \exp \left[\lambda^{-2}\left(t \cdot e^{\eta}-\frac{\eta^{2}}{2}\right)\right] .
$$

The critical point $\eta=T$ of the function $f(\eta)=t \cdot e^{\eta}-\frac{\eta^{2}}{2}$ is given by

$$
T=t \cdot e^{T},
$$

whose solution may be explicitly given by the Lambert series [10]:

$$
T=\sum_{d=1}^{\infty} \frac{d^{d-1}}{d !} t^{d} .
$$

Write $x=\eta-T$, then the expansion $f(\eta)$ at $T$ is

$$
\begin{aligned}
f(\eta) & =\left(t e^{T}-\frac{T^{2}}{2}\right)+\frac{t e^{T}-1}{2} x^{2}+\sum_{n=3}^{\infty} \frac{t e^{T}}{n !} x^{n} \\
& =\left(T-\frac{T^{2}}{2}\right)+\frac{T-1}{2} x^{2}+\sum_{n=3}^{\infty} \frac{T}{n !} x^{n} .
\end{aligned}
$$

Therefore in this case, the propagator is

$$
\kappa=\frac{1}{1-T},
$$

and the point on the space of coupling constants is specified by

$$
I_{0}=T ; \quad I_{k}=T, \quad k \geq 1
$$

or in the coordinates $\left\{t_{k}\right\}$,

$$
t_{k}=t, \quad k \geq 0
$$


By (7.12), the free energy is

$$
F_{g}^{\mathrm{gr}}=\sum_{\Gamma \in \mathcal{G}_{g, 0}^{c, 0}} \frac{1}{|\operatorname{Aut}(\Gamma)|} \cdot \frac{T^{|V(\Gamma)|}}{(1-T)^{|E(\Gamma)|}} .
$$

For example,

$$
\begin{aligned}
F_{0}^{\mathrm{gr}}= & T-\frac{T^{2}}{2}, \quad F_{1}^{\mathrm{gr}}=\frac{1}{2} \log \frac{1}{1-T}, \\
F_{2}^{\mathrm{gr}}= & \frac{T}{8(1-T)^{2}}+\frac{5 T^{2}}{24(1-T)^{3}}, \\
F_{3}^{\mathrm{gr}}= & \frac{T}{48(1-T)^{3}}+\frac{11 T^{2}}{48(1-T)^{4}}+\frac{25 T^{3}}{48(1-T)^{5}}+\frac{5 T^{4}}{16(1-T)^{6}}, \\
F_{4}^{\mathrm{gr}}= & \frac{T}{384(1-T)^{4}}+\frac{223 T^{2}}{1920(1-T)^{5}}+\frac{515 T^{3}}{576(1-T)^{6}}+\frac{1373 T^{4}}{576(1-T)^{7}} \\
& +\frac{985 T^{5}}{384(1-T)^{8}}+\frac{1105 T^{6}}{1152(1-T)^{9}} .
\end{aligned}
$$

These match with $\mathcal{G}_{k}$ in $[10,(63)]$.

By comparing (7.60) with (7.52), it is clear that for $g \geq 2$,

$$
F_{g}^{\mathrm{gr}}=F_{g}^{s t}\left(T, \frac{1}{1-T}\right) \text {. }
$$

Therefore, for $g \geq 2$ the free energy $F_{g}^{g r}$ can be written in the form

$$
F_{g}^{g r}=\sum_{d=1}^{2 g-2} \frac{a_{d}^{g} \cdot T^{d}}{(1-T)^{d+g-1}} .
$$

Moreover, note that for $g \geq 2, F_{g}^{g r}$ is of the form

$$
F_{g}^{\mathrm{gr}}=\frac{f_{g}(T)}{(1-T)^{3 g-3}},
$$

where $f_{g}(T)$ is a polynomial of degree $\leq 2 g-2$ without constant term. For example,

$$
\begin{aligned}
F_{2}^{\mathrm{gr}}= & \frac{T}{24(1-T)^{3}}(3+2 T), \\
F_{3}^{\mathrm{gr}}= & \frac{T}{48(1-T)^{6}}\left(1+8 T+6 T^{2}\right), \\
F_{4}^{\mathrm{gr}}= & \frac{T}{5760(1-T)^{9}}\left(15+594 T+2624 T^{2}+2144 T^{3}+164 T^{4}-16 T^{5}\right), \\
F_{5}^{\mathrm{gr}}= & \frac{T}{11520(1-T)^{12}}\left(3+465 T+6730 T^{2}+21940 T^{3}+18940 T^{4}\right. \\
& \left.+3012 T^{5}-240 T^{6}\right), \\
F_{6}^{\mathrm{gr}}= & \frac{T}{2903040(1-T)^{15}}\left(63+35568 T+1349298 T^{2}+11582816 T^{3}+31178616 T^{4}\right. \\
& \left.+27897072 T^{5}+6526912 T^{6}-266448 T^{7}-36576 T^{8}+2304 T^{9}\right) .
\end{aligned}
$$


Let

$$
f_{g}(T)=\sum_{n=1}^{2 g-2} b_{n}^{g} \cdot T^{n},
$$

then the two type of coefficients $\left\{a_{k}^{g}\right\}$ and $\left\{b_{k}^{g}\right\}$ are related by

$$
b_{k}^{g}=\sum_{l=1}^{k}(-1)^{k+l}\left(\begin{array}{c}
2 g-2-l \\
k-l
\end{array}\right) a_{l}^{g} .
$$

In particular,

$$
\begin{aligned}
b_{1}^{g} & =a_{1}^{g}=\frac{1}{(2 g) ! !}, \\
b_{2}^{g} & =a_{2}^{g}-(2 g-3) a_{1}^{g} \\
& =\frac{2^{2 g}-2^{g-1}-(g+1)^{2}}{2^{g} \cdot(g+1) !}-\frac{2 g-3}{(2 g) ! !} .
\end{aligned}
$$

As pointed out in $[10,(64),(65)], F_{g}^{\mathrm{gr}}$ is of the form $F_{g}^{\mathrm{gr}}=\sum_{n=g-1}^{3 g-3} \lambda_{g, n} \kappa^{n}$, and

$$
\lambda_{g, g-1}=\frac{B_{g}}{g(g-1)}, \quad \lambda_{g, 3 g-3}=b_{g-1},
$$

where $B_{g}$ are the Bernoulli numbers, and $b_{g}$ are given by

$$
\sum_{n=1}^{\infty} b_{r} x^{r}=\log \left(\sum_{m=0}^{\infty} \frac{(6 m) !}{(3 m) !(2 m) !}\left(\frac{x}{288}\right)^{m}\right) .
$$

The numbers $\left\{b_{g}\right\}$ are exactly the coefficients appearing in the expansion of the Airy function (7.41). Here are some examples of $F_{g}^{g r}$ written as polynomials in $\kappa$ :

$$
\begin{aligned}
F_{2}^{\mathrm{gr}}= & \frac{5}{24} \kappa^{3}-\frac{7}{24} \kappa^{2}+\frac{1}{12} \kappa \\
F_{3}^{\mathrm{gr}}= & \frac{5}{16} \kappa^{6}-\frac{35}{48} \kappa^{5}+\frac{13}{24} \kappa^{4}-\frac{1}{8} \kappa^{3}, \\
F_{4}^{\mathrm{gr}}= & \frac{1105}{1152} \kappa^{9}-\frac{1225}{384} \kappa^{8}+\frac{2273}{576} \kappa^{7}-\frac{313}{144} \kappa^{6}+\frac{227}{480} \kappa^{5}-\frac{17}{1440} \kappa^{4}-\frac{1}{360} \kappa^{3}, \\
F_{5}^{\mathrm{gr}}= & \frac{565}{128} \kappa^{12}-\frac{14665}{768} \kappa^{11}+\frac{76367}{2304} \kappa^{10}-\frac{11191}{384} \kappa^{9}+\frac{2557}{192} \kappa^{8}-\frac{7993}{2880} \kappa^{7} \\
& +\frac{37}{320} \kappa^{6}+\frac{1}{48} \kappa^{5}, \\
F_{6}^{\mathrm{gr}}= & \frac{82825}{3072} \kappa^{15}-\frac{441245}{3072} \kappa^{14}+\frac{493235}{1536} \kappa^{13}-\frac{16116187}{41472} \kappa^{12}+\frac{2827135}{10368} \kappa^{11} \\
& -\frac{1884983}{17280} \kappa^{10}+\frac{567289}{25920} \kappa^{9}-\frac{7489}{6480} \kappa^{8}-\frac{10249}{60480} \kappa^{7}+\frac{47}{10080} \kappa^{6}+\frac{1}{1260} \kappa^{5}, \\
F_{7}^{\mathrm{gr}}= & \frac{19675}{96} \kappa^{18}-\frac{7969325}{6144} \kappa^{17}+\frac{65405005}{18432} \kappa^{16}-\frac{453853985}{82944} \kappa^{15} \\
& +\frac{215237149}{41472} \kappa^{14}-\frac{64035527}{20736} \kappa^{13}+\frac{23126555}{20736} \kappa^{12}-\frac{11204309}{51840} \kappa^{11} \\
& +\frac{1352989}{103680} \kappa^{10}+\frac{6481}{4032} \kappa^{9}-\frac{1927}{20160} \kappa^{8}-\frac{1}{72} \kappa^{7} .
\end{aligned}
$$


Plug $T=1-\frac{1}{\kappa}$ into (7.54), we get the generating series for $\left\{\lambda_{g, k}\right\}$ :

$$
\begin{aligned}
& \sum_{g=2}^{\infty} \lambda^{2 g-2} \sum_{d=g-1}^{3 g-3} \lambda_{g, d} \kappa^{d} \\
= & \log \left(\sum_{k \geq 0} \sum_{l_{i} \geq 3} \sum_{l} \frac{\lambda^{2 l-2 k}}{k ! \cdot l_{1} ! \cdots l_{k} !} \cdot(2 l-1) ! ! \cdot(\kappa-1)^{k} \kappa^{l-k} \cdot \delta_{l_{1}+\cdots+l_{k}-2 l, 0}\right) .
\end{aligned}
$$

The relations among $\left\{a_{k}^{g}\right\},\left\{b_{k}^{g}\right\}$ and $\left\{\lambda_{g, k}\right\}$ are given by (7.69) and

$$
\begin{aligned}
\lambda_{g, 3 g-3-k} & =(-1)^{k} \sum_{l=k}^{2 g-2}\left(\begin{array}{l}
l \\
k
\end{array}\right) b_{l}^{g}, \\
b_{n}^{g} & =(-1)^{n} \sum_{j=g-1}^{3 g-3}\left(\begin{array}{c}
3 g-3-j \\
n
\end{array}\right) \lambda_{g, j}, \\
\lambda_{g, 3 g-3-k} & =\sum_{l=0}^{k}(-1)^{k+l}\left(\begin{array}{c}
2 g-2-l \\
k-l
\end{array}\right) a_{2 g-2-l} .
\end{aligned}
$$

Now we apply the quadratic recursion relations for topological 1D developed in section 7.2 to this problem. For this purpose, we need to find an operator that satisfies the conditions in $(7.26)$. We can take $d_{X}$ to be $D=\frac{\partial}{\partial(\log T)}=T \frac{\partial}{\partial T}$, because we have

$$
I_{k}=T, \quad \kappa=\frac{1}{1-T},
$$

the conditions in (7.26) are satisfied by $D$. So we get the following equation from (7.25):

$$
\partial_{\kappa} F^{\mathrm{gr}}=\frac{1}{2}\left((D+\kappa T) D F_{g-1}^{\mathrm{gr}}+\sum_{r=1}^{g-1} D F_{r}^{\mathrm{gr}} D F_{g-r}^{\mathrm{gr}}\right),
$$

Lemma 7.3. For $g \geq 2$, we have

$$
\left(\kappa+\kappa^{2} T\right) \partial_{\kappa} F_{g}^{\mathrm{gr}}-D F_{g}^{\mathrm{gr}}=(g-1) F_{g}^{\mathrm{gr}} .
$$

Proof. Every monomial in $F_{g}^{\mathrm{gr}}$ for $g \geq 2$ is of the form $a_{g, k} T^{k} \kappa^{k+g-1}$. Then the lemma follows from

$$
\kappa \partial_{\kappa}\left(T^{k} \kappa^{k+g-1}\right)=(k+g-1) T^{k} \kappa^{k+g-1}
$$

and

$$
D\left(T^{k} \kappa^{k+g-1}\right)=k T^{k} \kappa^{k+g-1}+(k+g-1) T^{k} \kappa^{k+g-2} \cdot \kappa^{2} T .
$$

Since $\kappa+\kappa^{2} T=\frac{1}{(1-T)^{2}},(7.72)$ and Lemma 7.3 give us:

Theorem 7.6. For $g \geq 2$, we have

$$
(D+g-1) F_{g}^{\mathrm{gr}}=\frac{1}{2(1-T)^{2}}\left[\left(D+\frac{T}{1-T}\right) D F_{g-1}^{\mathrm{gr}}+\sum_{r=1}^{g-1}\left(D F_{r}^{\mathrm{gr}}\right)\left(D F_{g-r}^{\mathrm{gr}}\right)\right] .
$$


This theorem provides a way to compute $F_{g}^{\mathrm{gr}}$ recursively. In fact, the left-hand side of (7.74) can be rewritten as

$$
\begin{aligned}
(D+g-1) F_{g}^{\mathrm{gr}} & =T \frac{d}{d T} F_{g}^{\mathrm{gr}}(T)+(g-1) F_{g}^{\mathrm{gr}}(T) \\
& =T^{2-g} \frac{d}{d T}\left(T^{g-1} \cdot F_{g}^{\mathrm{gr}}(T)\right),
\end{aligned}
$$

thus $F_{g}^{g r}$ is determined by the formula

$$
F_{g}^{\mathrm{gr}}(T)=T^{1-g} \cdot \int_{0}^{T} \frac{T^{g-2}}{2(1-T)^{2}}\left[\left(D+\frac{T}{1-T}\right) D F_{g-1}^{\mathrm{gr}}+\sum_{r=1}^{g-1}\left(D F_{r}^{\mathrm{gr}}\right)\left(D F_{g-r}^{\mathrm{gr}}\right)\right] d T .
$$

Moreover, for $g \geq 2$, when $F_{g}^{g r}(T)$ is written as a polynomial in the propagator $\kappa=$ $(1-T)^{-1}$ using $T=1-\frac{1}{\kappa}$, the recursion relation (7.74) can be written as follows.

Theorem 7.7. For $g \geq 2$, we have

$$
\begin{aligned}
{\left[\left(\kappa^{2}-\kappa\right) \frac{d}{d \kappa}+g-1\right] F_{g}^{\mathrm{gr}}=\frac{\kappa^{2}}{2}[} & \left(\kappa^{2}-\kappa\right)^{2} \frac{d^{2} F_{g-1}^{\mathrm{gr}}}{d \kappa^{2}}+(3 \kappa-2)\left(\kappa^{2}-\kappa\right) \frac{d F_{g-1}^{\mathrm{gr}}}{d \kappa} \\
& \left.+\left(\kappa^{2}-\kappa\right)^{2} \sum_{r=1}^{g-1} \frac{d F_{r}^{\mathrm{gr}}}{d \kappa} \cdot \frac{d F_{g-r}^{\mathrm{gr}}}{d \kappa}\right]
\end{aligned}
$$

In particular, for $g \geq 3$,

$$
\begin{aligned}
{\left[\left(\kappa^{2}-\kappa\right) \frac{d}{d \kappa}+g-1\right] F_{g}^{\mathrm{gr}}=\frac{\kappa^{2}}{2} } & \left(\kappa^{2}-\kappa\right)^{2} \frac{d^{2} F_{g-1}^{\mathrm{gr}}}{d \kappa^{2}}+(4 \kappa-3)\left(\kappa^{2}-\kappa\right) \frac{d F_{g-1}^{\mathrm{gr}}}{d \kappa} \\
& \left.+\left(\kappa^{2}-\kappa\right)^{2} \sum_{r=2}^{g-2} \frac{d F_{r}^{\mathrm{gr}}}{d \kappa} \cdot \frac{d F_{g-r}^{\mathrm{gr}}}{d \kappa}\right]
\end{aligned}
$$

The equation (7.78) can be rewritten as

$$
\begin{gathered}
\frac{d}{d \kappa}\left((1-\kappa)^{g-1} \frac{F_{g}^{\mathrm{gr}}}{\kappa^{g-1}}\right) \\
=-\frac{1}{\kappa^{2}}\left(\frac{1}{\kappa}-1\right)^{g-2}\left[(g-1) F_{g}^{\mathrm{gr}}+\left(\kappa^{2}-\kappa\right) \frac{d F_{g}^{\mathrm{gr}}}{d \kappa}\right] \\
=-\frac{1}{2} \frac{(1-\kappa)^{g-2}}{\kappa^{g-2}}\left[\left(\kappa^{2}-\kappa\right)^{2} \frac{d^{2} F_{g-1}^{\mathrm{gr}}}{d \kappa^{2}}+(4 \kappa-3)\left(\kappa^{2}-\kappa\right) \frac{d F_{g-1}^{\mathrm{gr}}}{d \kappa}\right. \\
\left.+\left(\kappa^{2}-\kappa\right)^{2} \sum_{r=2}^{g-2} \frac{d F_{r}^{\mathrm{gr}}}{d \kappa} \cdot \frac{d F_{g-r}^{\mathrm{gr}}}{d \kappa}\right] .
\end{gathered}
$$

Now if we want to integrate this equality with respect to $\kappa$, we need to determine the constant of integration. Note that $(1-\kappa)^{g-1} \frac{F_{g}^{\mathrm{gr}}}{\kappa^{g-1}}$ is a polynomial in $\kappa$ whose constant term is $\lambda_{g, g-1}=\frac{B_{g}}{g(g-1)}$, therefore

$$
\begin{aligned}
F_{g}^{\mathrm{gr}}=\frac{\kappa^{g-1}}{(1-\kappa)^{g-1}}\left\{\frac{B_{g}}{g(g-1)}+\int_{0}^{\kappa}\left[-\frac{1}{2} \frac{(1-\kappa)^{g-2}}{\kappa^{g-2}}\left(\left(\kappa^{2}-\kappa\right)^{2} \frac{d^{2} F_{g-1}^{\mathrm{gr}}}{d \kappa^{2}}\right.\right.\right. \\
\left.\left.\left.\quad+(4 \kappa-3)\left(\kappa^{2}-\kappa\right) \frac{d F_{g-1}^{\mathrm{gr}}}{d \kappa}+\left(\kappa^{2}-\kappa\right)^{2} \sum_{r=2}^{g-2} \frac{d F_{r}^{\mathrm{gr}}}{d \kappa} \cdot \frac{d F_{g-r}^{\mathrm{gr}}}{d \kappa}\right)\right] d \kappa\right\}
\end{aligned}
$$


Now let us derive some recursion relations for the coefficients $\left\{\lambda_{g, k}\right\}$ using Theorem 7.7. Expand the two sides of (7.78), we get

$$
\begin{aligned}
& \sum_{l}[l(\kappa-1)+(g-1)] \lambda_{g, l} \kappa^{l} \\
&=\frac{\kappa^{2}}{2}\left[\sum_{l}(\kappa-1)^{2} l(l-1) \lambda_{g-1, l} \kappa^{l}+\sum_{l}(4 \kappa-3)(\kappa-1) l \lambda_{g-1, l} \kappa^{l}\right. \\
&\left.\quad+\sum_{r=2}^{g-2} \sum_{l_{1}, l_{2}}(\kappa-1)^{2} l_{1} l_{2} \lambda_{r, l_{1}} \lambda_{g-r, l_{2}} \kappa^{l_{1}+l_{2}}\right],
\end{aligned}
$$

which gives us

$$
\begin{aligned}
& (l-1) \lambda_{g, l-1}+(g-l-1) \lambda_{g, l} \\
= & \frac{1}{2}(l-4)(l-1) \lambda_{g-1, l-4}-\frac{1}{2}(l-3)(2 l-1) \lambda_{g-1, l-3}+\frac{1}{2}(l-2) l \lambda_{g-1, l-2} \\
+ & \frac{1}{2} \sum_{r=2}^{g-2}\left(\sum_{l_{1}+l_{2}=l-4} l_{1} l_{2} \lambda_{r, l_{1}} \lambda_{g-r, l_{2}}-2 \sum_{l_{1}+l_{2}=l-3} l_{1} l_{2} \lambda_{r, l_{1}} \lambda_{g-r, l_{2}}\right. \\
& \left.+\sum_{l_{1}+l_{2}=l-2} l_{1} l_{2} \lambda_{r, l_{1}} \lambda_{g-r, l_{2}}\right) .
\end{aligned}
$$

Note that $\lambda_{g, n}=0$ unless $g-1 \leq n \leq 3 g-3$, thus the above relation indeed determines $\left\{\lambda_{g, n}\right\}$ uniquely. In fact, first we set $l=3 g-2$, then (7.81) gives

$$
\begin{aligned}
\lambda_{g, 3 g-3}= & \frac{1}{2}(3 g-6) \lambda_{g-1,3 g-6}-\frac{(3 g-5)(6 g-5)}{2(3 g-3)} \lambda_{g-1,3 g-5} \\
& +\frac{(3 g-4)(3 g-2)}{2(3 g-3)} \lambda_{g-1,3 g-4}+\frac{1}{2(3 g-3)} \sum_{r=2}^{g-2}\left(\sum_{l_{1}+l_{2}=3 g-6} l_{1} l_{2} \lambda_{r, l_{1}} \lambda_{g-r, l_{2}}\right. \\
& \left.-2 \sum_{l_{1}+l_{2}=3 g-5} l_{1} l_{2} \lambda_{r, l_{1}} \lambda_{g-r, l_{2}}+\sum_{l_{1}+l_{2}=3 g-4} l_{1} l_{2} \lambda_{r, l_{1}} \lambda_{g-r, l_{2}}\right) .
\end{aligned}
$$

Then set $l=3 g-3,(7.81)$ gives

$$
\begin{aligned}
& (3 g-4) \lambda_{g, 3 g-4}+(2-2 g) \lambda_{g, 3 g-3} \\
= & \frac{1}{2}(3 g-7)(3 g-4) \lambda_{g-1,3 g-7}-\frac{1}{2}(3 g-6)(6 g-7) \lambda_{g-1,3 g-6} \\
& +\frac{1}{2}(3 g-5) l \lambda_{g-1,3 g-5}+\frac{1}{2} \sum_{r=2}^{g-2}\left(\sum_{l_{1}+l_{2}=3 g-7} l_{1} l_{2} \lambda_{r, l_{1}} \lambda_{g-r, l_{2}}\right. \\
& \left.-2 \sum_{l_{1}+l_{2}=3 g-6} l_{1} l_{2} \lambda_{r, l_{1}} \lambda_{g-r, l_{2}}+\sum_{l_{1}+l_{2}=3 g-5} l_{1} l_{2} \lambda_{r, l_{1}} \lambda_{g-r, l_{2}}\right),
\end{aligned}
$$

from which we can solve $\lambda_{g, 3 g-4}$ using $\lambda_{g, 3 g-3}$ and lower genus $\lambda_{r, n}$. Similarly $\lambda_{g, n}$ can be solved recursively from $n=3 g-3$ to $n=g-1$ using (7.81). 


\section{Acknowledgments}

We thank an anonymous referee for helpful suggestions that improve the presentation of this paper. The second author is partly supported by NSFC grant 11661131005 .

\section{A Some explicit expressions for $\widehat{\boldsymbol{F}}_{g, n}$}

In this appendix, we give more examples of the abstract free energies.

For the case $N=1$, here are the explicit expressions for $\widehat{\mathcal{F}}_{2,2}$ and $\widehat{\mathcal{F}}_{3}$ in terms of Feynman graphs.

$$
\begin{aligned}
& \widehat{\mathcal{F}}_{2,2}=\frac{1}{2} \text { (2) }+\frac{1}{4} \text { C(1) }+\frac{1}{2}-\text { (1)-(1)- }+\frac{1}{2} \text { (2)-(1) }+\frac{1}{2} \text { (1)-(1) }+\frac{1}{16} \text { C(1) } \\
& +\frac{1}{4} \text { (1)-(0) }+\frac{1}{2} \text { C(0)-(1)- }+\frac{1}{2}-\text { (0)-(1)- }+\frac{1}{4} \text { C(0)-(1) } 2+\frac{1}{4} \text { C(1)-(0) }
\end{aligned}
$$

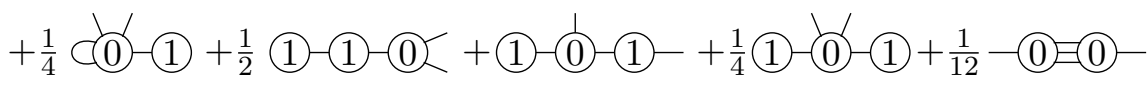

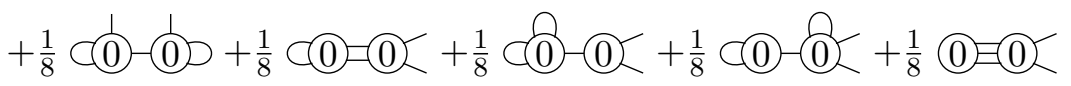

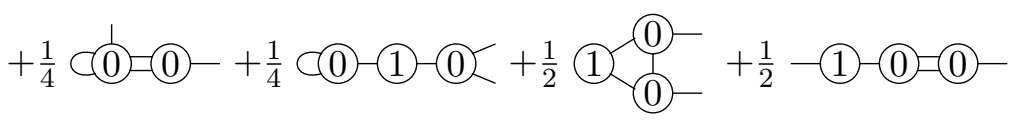

$$
\begin{aligned}
& +\frac{1}{2} \text { C(0)-(0)-(1)- + } \frac{1}{4} \text { (1)=(0)-(0) }+\frac{1}{2} \text { C(0)-(0)-(1) }+\frac{1}{4} \text { (1)-(0)-(0) }
\end{aligned}
$$

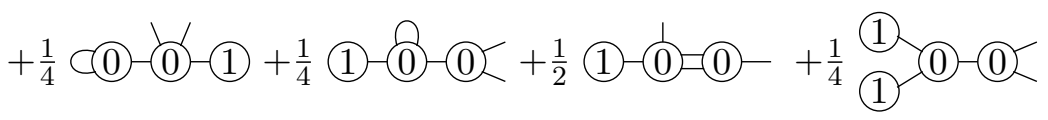

$$
\begin{aligned}
& +\frac{1}{2} \text { (1)-(0)-(0)-(1) + } \frac{1}{8} \text { C(0)=(0)-(0 }<+\frac{1}{8} \text { C(0)-(0)=(0) }+\frac{1}{4} \text { C(0)-(0)-(0) } \\
& +\frac{1}{8} \text { C(0)-(0)-(0) }+\frac{1}{16} \text { C(0)-(0)-(0) }+\frac{1}{4} \text { C(0)-(0)=-(0)- + } \frac{1}{12} \text { (0)=(0)-(0) } \\
& +\frac{1}{4} \text { C(0) (0)- }+\frac{1}{4} \text { C(0)-(0)=(0)- + }+\frac{1}{8} \text {-(0) (0) }+\frac{1}{8}-\text { (0)=(0)-(0)- }
\end{aligned}
$$

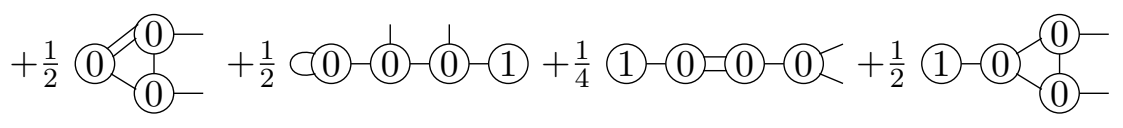

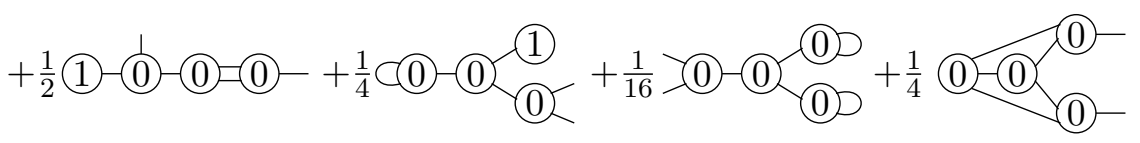

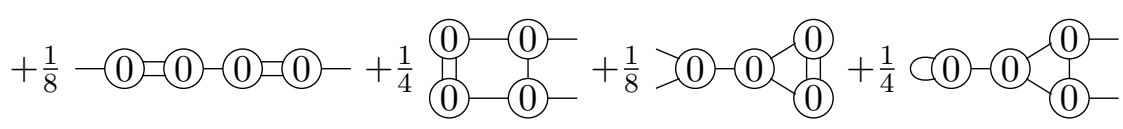

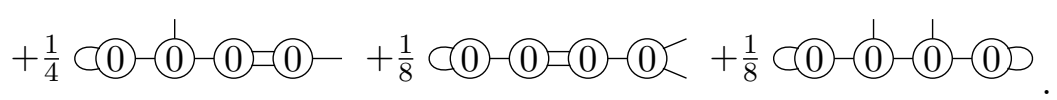




$$
\begin{aligned}
& \widehat{\mathcal{F}}_{3}=(3)+\frac{1}{2}(2)+(2)-(1)+\frac{1}{8}(1)+\frac{1}{4}(1)-(1)+\frac{1}{2}(2)-(0)+\frac{1}{2} \text { (1)-(1) } \\
& +\frac{1}{2} \text { (1)-(1)-(1) }+\frac{1}{48} \text { C(0) }+\frac{1}{4} \text { (1)-(0) }+\frac{1}{6} \text { (1)=(0) }+\frac{1}{4} \text { C(1)-(0) } \\
& +\frac{1}{8} \text { (1)-(0) + } \frac{1}{2} \text { (1)-(1)-(0) + } \frac{1}{2} \text { (1)-(0)=(1) + } \frac{1}{4} \text { (1)-(0)-(1) + } \frac{1}{6} \text { (1)-(0) (1) } \\
& +\frac{1}{48} \text { (1)=(0) }+\frac{1}{16} C(0)=(0)+\frac{1}{16} \text { C(0)-(0) }+\frac{1}{12} C(0)=(0)+\frac{1}{8} \text { C(0)-(1)-(0) } \\
& +\frac{1}{4} \text { (1) (0) }+\frac{1}{4} \text { (1)-(0)=(0) + } \frac{1}{4} \text { (1)=(0)-(0) + } \frac{1}{4}(1)-(0)-(0)+\frac{1}{6} \text { (1)-(0)=(0) } \\
& +\frac{1}{4} \text { (1)-(0) }(1)+\frac{1}{4} \text { (1)-(0)-(0)-(1) }+\frac{1}{8} \text { C(0)-(0)-(0) }+\frac{1}{16} \text { C(0)-(0)-(0) } \\
& +\frac{1}{12} \text { (1)-(0)=(0) }+\frac{1}{8} \text { C(0) (0) }+\frac{1}{8} \text { (0) (0) }+\frac{1}{4} \text { (1)-(0)=(0)-(0) }
\end{aligned}
$$

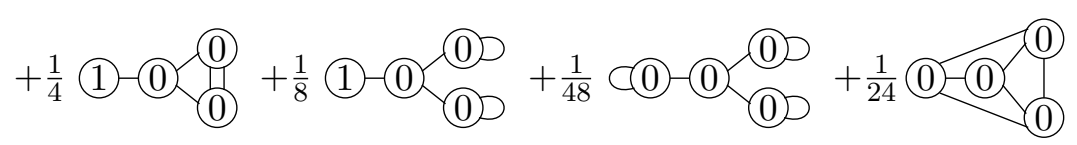

$$
\begin{aligned}
& +\frac{1}{16} \frac{(0)=(0)}{\text { (0)-(0) }}+\frac{1}{16} \text { C(0)-(0)-(0)-(0) }+\frac{1}{8} \text { C(0)-(0) (0) }
\end{aligned}
$$

And in terms of the derivatives of the holomorphic free energy $F(t)$ together with the propagator $\kappa$, the explicit expressions for $\widehat{F}_{3}$ and $\widehat{F}_{4}$ are as follows.

$$
\begin{aligned}
\widehat{F}_{3}= & F_{3}+\left(\frac{1}{2} F_{2}^{\prime \prime}+F_{1}^{\prime} F_{2}^{\prime}\right) \kappa \\
+ & {\left[\frac{1}{8} F_{1}^{(4)}+\frac{1}{4}\left(F_{1}^{\prime \prime}\right)^{2}+\frac{1}{2} F_{0}^{\prime \prime \prime} F_{2}^{\prime}+\frac{1}{2} F_{1}^{\prime} F_{1}^{\prime \prime \prime}+\frac{1}{2}\left(F_{1}^{\prime}\right)^{2} F_{1}^{\prime \prime}\right] \kappa^{2} } \\
+ & {\left[\frac{1}{48} F_{0}^{(6)}+\frac{1}{4} F_{0}^{(4)} F_{1}^{\prime \prime}+\frac{5}{12} F_{0}^{\prime \prime \prime} F_{1}^{\prime \prime \prime}+\frac{1}{8} F_{0}^{(5)} F_{1}^{\prime}+F_{0}^{\prime \prime \prime} F_{1}^{\prime} F_{1}^{\prime \prime}\right.} \\
& \left.+\frac{1}{4} F_{0}^{(4)}\left(F_{1}^{\prime}\right)^{2}+\frac{1}{6} F_{0}^{\prime \prime \prime}\left(F_{1}^{\prime}\right)^{3}\right] \kappa^{3} \\
+ & {\left[\frac{1}{12}\left(F_{0}^{(4)}\right)^{2}+\frac{7}{48} F_{0}^{\prime \prime \prime} F_{0}^{(5)}+\frac{5}{8}\left(F_{0}^{\prime \prime \prime}\right)^{2} F_{1}^{\prime \prime}+\frac{2}{3} F_{0}^{\prime \prime \prime} F_{0}^{(4)} F_{1}^{\prime}+\frac{1}{2}\left(F_{0}^{\prime \prime \prime}\right)^{2}\left(F_{1}^{\prime}\right)^{2}\right] \kappa^{4} } \\
+ & {\left[\frac{25}{48}\left(F_{0}^{\prime \prime \prime}\right)^{2} F_{0}^{(4)}+\frac{5}{8}\left(F_{0}^{\prime \prime \prime}\right)^{3} F_{1}^{\prime}\right] \kappa^{5}+\frac{5}{16}\left(F_{0}^{\prime \prime \prime}\right)^{4} \kappa^{6} . }
\end{aligned}
$$




$$
\begin{aligned}
& \widehat{F}_{4}=F_{4}+\left[\frac{1}{2}\left(F_{2}^{\prime}\right)^{2}+F_{1}^{\prime} F_{3}^{\prime}+\frac{1}{2} F_{3}^{\prime \prime}\right] \kappa \\
& +\left[F_{1}^{\prime} F_{1}^{\prime \prime} F_{2}^{\prime}+\frac{1}{2}\left(F_{1}^{\prime}\right)^{2} F_{2}^{\prime \prime}+\frac{1}{2} F_{1}^{\prime \prime} F_{2}^{\prime \prime}+\frac{1}{2} F_{0}^{\prime \prime \prime} F_{3}^{\prime}+\frac{1}{2} F_{1}^{\prime \prime \prime} F_{2}^{\prime}+\frac{1}{2} F_{1}^{\prime} F_{2}^{\prime \prime \prime}+\frac{1}{8} F_{2}^{(4)}\right] \kappa^{2} \\
& +\left[\frac{1}{2}\left(F_{1}^{\prime}\right)^{2}\left(F_{1}^{\prime \prime}\right)^{2}+\frac{1}{6}\left(F_{1}^{\prime \prime}\right)^{3}+\frac{1}{2} F_{0}^{\prime \prime \prime}\left(F_{1}^{\prime}\right)^{2} F_{2}^{\prime}+F_{0}^{\prime \prime \prime} F_{1}^{\prime \prime} F_{2}^{\prime}+F_{0}^{\prime \prime \prime} F_{1}^{\prime} F_{2}^{\prime \prime}\right. \\
& +\frac{1}{6}\left(F_{1}^{\prime}\right)^{3} F_{1}^{\prime \prime \prime}+F_{1}^{\prime} F_{1}^{\prime \prime} F_{1}^{\prime \prime \prime}+\frac{5}{24}\left(F_{1}^{\prime \prime \prime}\right)^{2}+\frac{5}{12} F_{0}^{\prime \prime \prime} F_{2}^{\prime \prime \prime}+\frac{1}{2} F_{0}^{(4)} F_{1}^{\prime} F_{2}^{\prime} \\
& \left.+\frac{1}{4} F_{0}^{(4)} F_{2}^{\prime \prime}+\frac{1}{4}\left(F_{1}^{\prime}\right)^{2} F_{1}^{(4)}+\frac{1}{4} F_{1}^{\prime \prime} F_{1}^{(4)}+\frac{1}{8} F_{0}^{(5)} F_{2}^{\prime}+\frac{1}{8} F_{1}^{(5)} F_{1}^{\prime}+\frac{1}{48} F_{1}^{(6)}\right] \kappa^{3} \\
& +\left[\frac{1}{2} F_{0}^{\prime \prime \prime}\left(F_{1}^{\prime}\right)^{3} F_{1}^{\prime \prime}+\frac{3}{2} F_{0}^{\prime \prime \prime} F_{1}^{\prime}\left(F_{1}^{\prime \prime}\right)^{2}+\left(F_{0}^{\prime \prime \prime}\right)^{2} F_{1}^{\prime} F_{2}^{\prime}+\frac{5}{8}\left(F_{0}^{\prime \prime \prime}\right)^{2} F_{2}^{\prime \prime}+F_{0}^{\prime \prime \prime}\left(F_{1}^{\prime}\right)^{2} F_{1}^{\prime \prime \prime}\right. \\
& +\frac{5}{4} F_{0}^{\prime \prime \prime} F_{1}^{\prime \prime} F_{1}^{\prime \prime \prime}+\frac{1}{24} F_{0}^{(4)}\left(F_{1}^{\prime}\right)^{4}+\frac{3}{4} F_{0}^{(4)}\left(F_{1}^{\prime}\right)^{2} F_{1}^{\prime \prime}+\frac{3}{8} F_{0}^{(4)}\left(F_{1}^{\prime \prime}\right)^{2} \\
& +\frac{2}{3} F_{0}^{\prime \prime \prime} F_{0}^{(4)} F_{2}^{\prime}+\frac{2}{3} F_{0}^{(4)} F_{1}^{\prime} F_{1}^{\prime \prime \prime}+\frac{2}{3} F_{0}^{\prime \prime \prime} F_{1}^{\prime} F_{1}^{(4)}+\frac{1}{6} F_{0}^{(4)} F_{1}^{(4)}+\frac{1}{12} F_{0}^{(5)}\left(F_{1}^{\prime}\right)^{3} \\
& +\frac{3}{8} F_{0}^{(5)} F_{1}^{\prime} F_{1}^{\prime \prime}+\frac{7}{48} F_{0}^{(5)} F_{1}^{\prime \prime \prime}+\frac{7}{48} F_{0}^{\prime \prime \prime} F_{1}^{(5)}+\frac{1}{16} F_{0}^{(6)}\left(F_{1}^{\prime}\right)^{2}+\frac{1}{16} F_{0}^{(6)} F_{1}^{\prime \prime} \\
& \left.+\frac{1}{48} F_{0}^{(7)} F_{1}^{\prime}+\frac{1}{384} F_{0}^{(8)}\right] \kappa^{4} \\
& +\left[\frac{1}{8}\left(F_{0}^{\prime \prime \prime}\right)^{2}\left(F_{1}\right)^{4}+2\left(F_{0}^{\prime \prime \prime}\right)^{2}\left(F_{1}^{\prime}\right)^{2} F_{1}^{\prime \prime}+\frac{5}{4}\left(F_{0}^{\prime \prime \prime}\right)^{2}\left(F_{1}^{\prime \prime}\right)^{2}+\frac{5}{8}\left(F_{0}^{\prime \prime \prime}\right)^{3} F_{2}^{\prime}\right. \\
& +\frac{15}{8}\left(F_{0}^{\prime \prime \prime}\right)^{2} F_{1}^{\prime} F_{1}^{\prime \prime \prime}+\frac{7}{12} F_{0}^{\prime \prime \prime} F_{0}^{(4)}\left(F_{1}^{\prime}\right)^{3}+\frac{8}{3} F_{0}^{\prime \prime \prime} F_{0}^{(4)} F_{1}^{\prime} F_{1}^{\prime \prime}+\frac{25}{24} F_{0}^{\prime \prime \prime} F_{0}^{(4)} F_{1}^{\prime \prime \prime} \\
& +\frac{1}{3}\left(F_{0}^{(4)}\right)^{2}\left(F_{1}^{\prime}\right)^{2}+\frac{1}{3}\left(F_{0}^{(4)}\right)^{2} F_{1}^{\prime \prime}+\frac{25}{48}\left(F_{0}^{\prime \prime \prime}\right)^{2} F_{1}^{(4)}+\frac{25}{48} F_{0}^{\prime \prime \prime} F_{0}^{(5)}\left(F_{1}^{\prime}\right)^{2} \\
& +\frac{7}{12} F_{0}^{\prime \prime \prime} F_{0}^{(5)} F_{1}^{\prime \prime}+\frac{5}{16} F_{0}^{(4)} F_{0}^{(5)} F_{1}^{\prime}+\frac{21}{640}\left(F_{0}^{(5)}\right)^{2}+\frac{5}{24} F_{0}^{\prime \prime \prime} F_{0}^{(6)} F_{1}^{\prime} \\
& \left.+\frac{5}{96} F_{0}^{(4)} F_{0}^{(6)}+\frac{1}{32} F_{0}^{\prime \prime \prime} F_{0}^{(7)}\right] \kappa^{5} \\
& +\left[\frac{2}{3}\left(F_{0}^{\prime \prime \prime}\right)^{3}\left(F_{1}^{\prime}\right)^{3}+\frac{25}{8}\left(F_{0}^{\prime \prime \prime}\right)^{3} F_{1}^{\prime} F_{1}^{\prime \prime}+\frac{5}{4}\left(F_{0}^{\prime \prime \prime}\right)^{3} F_{1}^{\prime \prime \prime}+\frac{109}{48}\left(F_{0}^{\prime \prime \prime}\right)^{2} F_{0}^{(4)}\left(F_{1}^{\prime}\right)^{2}\right. \\
& +\frac{125}{48}\left(F_{0}^{\prime \prime \prime}\right)^{2} F_{0}^{(4)} F_{1}^{\prime \prime}+\frac{11}{8} F_{0}^{\prime \prime \prime}\left(F_{0}^{(4)}\right)^{2} F_{1}^{\prime}+\frac{11}{96}\left(F_{0}^{(4)}\right)^{3}+\frac{53}{48}\left(F_{0}^{\prime \prime \prime}\right)^{2} F_{0}^{(5)} F_{1}^{\prime} \\
& \left.+\frac{7}{12} F_{0}^{\prime \prime \prime} F_{0}^{(4)} F_{0}^{(5)}+\frac{113}{576}\left(F_{0}^{\prime \prime \prime}\right)^{2} F_{0}^{(6)}\right] \kappa^{6} \\
& +\left[\frac{25}{16}\left(F_{0}^{\prime \prime \prime}\right)^{4}\left(F_{1}^{\prime}\right)^{2}+\frac{15}{8}\left(F_{0}^{\prime \prime \prime}\right)^{4} F_{1}^{\prime \prime}+\frac{185}{48}\left(F_{0}^{\prime \prime \prime}\right)^{3} F_{0}^{(4)} F_{1}^{\prime}+\frac{445}{288}\left(F_{0}^{\prime \prime \prime}\right)^{2}\left(F_{0}^{(4)}\right)^{2}\right. \\
& \left.+\frac{161}{192}\left(F_{0}^{\prime \prime \prime}\right)^{3} F_{0}^{(5)}\right] \kappa^{7} \\
& +\left[\frac{15}{8}\left(F_{0}^{\prime \prime \prime}\right)^{5} F_{1}^{\prime}+\frac{985}{384}\left(F_{0}^{\prime \prime \prime}\right)^{4} F_{0}^{(4)}\right] \kappa^{8}+\frac{1105}{1152}\left(F_{0}^{\prime \prime \prime}\right)^{6} \kappa^{9} .
\end{aligned}
$$

In particular, by setting $F_{g}=0$ for all $g \geq 1$ and $F_{0}^{(k+1)}=I_{k}$, we recover the expressions (7.23) and (7.27) for $F_{3}^{1 \mathrm{D}}$ and $F_{4}^{1 \mathrm{D}}$. 
Open Access. This article is distributed under the terms of the Creative Commons Attribution License (CC-BY 4.0), which permits any use, distribution and reproduction in any medium, provided the original author(s) and source are credited.

\section{References}

[1] M. Aganagic, V. Bouchard and A. Klemm, Topological Strings and (Almost) Modular Forms, Commun. Math. Phys. 277 (2008) 771 [hep-th/0607100] [INSPIRE].

[2] M. Bershadsky, S. Cecotti, H. Ooguri and C. Vafa, Holomorphic anomalies in topological field theories, Nucl. Phys. B 405 (1993) 279 [hep-th/9302103] [INSPIRE].

[3] M. Bershadsky, S. Cecotti, H. Ooguri and C. Vafa, Kodaira-Spencer theory of gravity and exact results for quantum string amplitudes, Commun. Math. Phys. 165 (1994) 311 [hep-th/9309140] [INSPIRE].

[4] P. Candelas, X.C. De La Ossa, P.S. Green and L. Parkes, A Pair of Calabi-Yau manifolds as an exactly soluble superconformal theory, Nucl. Phys. B 359 (1991) 21 [INSPIRE].

[5] S. Cecotti, P. Fendley, K.A. Intriligator and C. Vafa, A New supersymmetric index, Nucl. Phys. B 386 (1992) 405 [hep-th/9204102] [INSPIRE].

[6] S. Cecotti and C. Vafa, Topological antitopological fusion, Nucl. Phys. B 367 (1991) 359 [INSPIRE].

[7] P. Deligne and D. Mumford, The irreducibility of the space of curves of given genus, Inst. Hautes Etudes Sci. Publ. Math. 36 (1969) 75.

[8] R. Dijkgraaf, H.L. Verlinde and E.P. Verlinde, Topological strings in $d<1$, Nucl. Phys. B 352 (1991) 59 [INSPIRE].

[9] B. Dubrovin, Geometry and integrability of topological - antitopological fusion, Commun. Math. Phys. 152 (1993) 539 [hep-th/9206037] [INSPIRE].

[10] B. Dubrovin, D. Yang and D. Zagier, Classical Hurwitz numbers and related combinatorics, Moscow Math. J. 17 (2017) 601.

[11] P. Dunin-Barkowski, N. Orantin, S. Shadrin and L. Spitz, Identification of the Givental formula with the spectral curve topological recursion procedure, Commun. Math. Phys. 328 (2014) 669 [arXiv:1211.4021] [inSPIRE].

[12] T. Eguchi, K. Hori and C.-S. Xiong, Quantum cohomology and Virasoro algebra, Phys. Lett. B 402 (1997) 71 [hep-th/9703086] [INSPIRE].

[13] B. Eynard, Intersection numbers of spectral curves, arXiv:1104.0176 [INSPIRE].

[14] B. Eynard, Invariants of spectral curves and intersection theory of moduli spaces of complex curves, Commun. Num. Theor. Phys. 8 (2014) 541 [arXiv:1110.2949] [InSPIRE].

[15] P.M. Chesler, A. Gynther and A. Vuorinen, On the dispersion of fundamental particles in QCD and $N=4$ Super Yang-Mills theory, JHEP 09 (2009) 003 [arXiv:0906.3052] [INSPIRE].

[16] B. Eynard and N. Orantin, Invariants of algebraic curves and topological expansion, Commun. Num. Theor. Phys. 1 (2007) 347 [math-ph/0702045] [INSPIRE].

[17] B. Eynard, M. Mariño and N. Orantin, Holomorphic anomaly and matrix models, JHEP 06 (2007) 058 [hep-th/0702110] [INSPIRE]. 
[18] E. Getzler, The Virasoro conjecture for Gromov-Witten invariants, Contemp. Math. 241 (1999) 147.

[19] T.W. Grimm, A. Klemm, M. Mariño and M. Weiss, Direct Integration of the Topological String, JHEP 08 (2007) 058 [hep-th/0702187] [INSPIRE].

[20] S. Gukov and P. Sułkowski, A-polynomial, B-model and Quantization, JHEP 02 (2012) 070 [arXiv: 1108.0002] [INSPIRE].

[21] J. Harer and D. Zagier, The Euler characteristic of the moduli space of curves, Invent. Math. 85 (1986) 457.

[22] F.F. Knudsen, The projectivity of the moduli space of stable curves, II: The stacks $M_{g, n}$, Math. Scand. 52 (1983) 161.

[23] M. Kontsevich, Intersection theory on the moduli space of curves and the matrix Airy function, Commun. Math. Phys. 147 (1992) 1 [INSPIRE].

[24] I. Kostov and N. Orantin, CFT and topological recursion, JHEP 11 (2010) 056 [arXiv: 1006.2028] [INSPIRE].

[25] M. Mulase and P. Sułkowski, Spectral curves and the Schrödinger equations for the Eynard-Orantin recursion, Adv. Theor. Math. Phys. 19 (2015) 955 [arXiv:1210.3006] [INSPIRE].

[26] S. Nishigaki and T. Yoneya, A nonperturbative theory of randomly branching chains, Nucl. Phys. B 348 (1991) 787 [INSPIRE].

[27] R.C. Penner, Perturbative series and the moduli space of Riemann surfaces, J. Diff. Geom. 27 (1988) 35 [inSPIRE].

[28] Z. Wang and J. Zhou, Orbifold Euler Characteristics of $\overline{\mathcal{M}}_{g, n}$, arXiv:1812.10638.

[29] Z. Wang and J. Zhou, in preparation.

[30] E. Witten, Quantum background independence in string theory, hep-th/9306122 [INSPIRE].

[31] E. Witten, Two-dimensional gravity and intersection theory on moduli space, Surveys Diff. Geom. 1 (1991) 243 [INSPIRE].

[32] M. Hayakawa and N. Ishibashi, Perturbative dynamics of matrix string for the membrane, JHEP 04 (2004) 047 [hep-th/0401227] [INSPIRE].

[33] J. Zhou, Intersection numbers on Deligne-Mumford moduli spaces and quantum Airy curve, arXiv:1206.5896 [INSPIRE].

[34] J. Zhou, On Topological 1D Gravity. I, arXiv:1412.1604 [INSPIRE].

[35] J. Zhou, Topological Recursions of Eynard-Orantin Type for Intersection Numbers on Moduli Spaces of Curves, Lett. Math. Phys. 103 (2013) 1191 [INSPIRE]. 